Supporting information for

\title{
Hydrogen production from Formic acid and Formaldehyde over Ruthenium Catalysts in Water
}

Soumyadip Patra ${ }^{\dagger}$ and Sanjay K. Singh*,†

${ }^{\dagger}$ Catalysis Group, Discipline of Chemistry, Indian Institute of Technology Indore, Simrol, Indore 453552, India

E-mail: sksingh@iiti.ac.in 
Table of Contents

\begin{tabular}{|c|c|}
\hline Description & Page no. \\
\hline Conversion, TON and TOF & S4 \\
\hline $\begin{array}{l}\text { Table S1. Comparative catalytic activity of previous literature work and } \\
\text { the current work for formic acid dehydrogenation. }\end{array}$ & S5-S6 \\
\hline Table S2. Single crystal X-ray refinement data for complex [Ru]-2 & S7 - S8 \\
\hline Table S3. Selected bond lengths $(\AA)$ for complex [Ru]-2 & S8 \\
\hline Table S4. Selected bond angles $(\AA)$ for complex [Ru]-2 & S8 - S9 \\
\hline $\begin{array}{l}\text { Table S5. Optimization of reaction condition for the catalytic } \\
\text { dehydrogenation of formic acid over [Ru]-1 in water at } 90^{\circ} \mathrm{C}\end{array}$ & S9 \\
\hline $\begin{array}{l}\text { Figure S1.TOF }\left(\mathrm{h}^{-1}\right) \text { vs [HCOONa] }(\mathrm{mmol}) \text { plot for the catalytic } \\
\text { dehydrogenation of formic acid over [Ru]-1. Reaction condition: formic } \\
\text { acid }(0.4 \mathrm{M}, 2.5 \mathrm{~mL}),[\mathrm{Ru}]-1(0.01 \mathrm{mmol}), 90^{\circ} \mathrm{C} \text {. }\end{array}$ & S10 \\
\hline $\begin{array}{l}\text { Figure S2. Gas produced (mmol) vs time (min) plot for the catalytic } \\
\text { dehydrogenation of formic acid over [Ru]-1. Reaction condition: formic } \\
\text { acid }(4.0 \mathrm{M}, 2.5 \mathrm{~mL}),[\mathrm{HCOONa}] /[\mathrm{HCOOH}]=2: 1 \text {, [Ru]-1 }(0.01 \mathrm{mmol}) \text {, } \\
90{ }^{\circ} \mathrm{C} \text {. }\end{array}$ & S10 \\
\hline Figures S3-S6. GC-TCD scans of different volumes of pure $\mathrm{H}_{2}$ gas & $\mathrm{S} 11-\mathrm{S} 12$ \\
\hline Figure S7. Calibration plot of $\mathrm{H}_{2}$ gas & S13 \\
\hline Figures S8-S11. GC-TCD scans of different volumes of pure $\mathrm{CO}_{2}$ gas & $\mathrm{S} 14-\mathrm{S} 15$ \\
\hline Figure S12. Calibration plot of $\mathrm{CO}_{2}$ gas & S16 \\
\hline $\begin{array}{l}\text { Figure S13. GC-TCD scan of blank sample using argon as the carrier } \\
\text { gas }\end{array}$ & S17 \\
\hline $\begin{array}{l}\text { Figure S14. GC-TCD analysis of (a) evolved gas }\left(\mathrm{H}_{2}: \mathrm{CO}_{2} \approx 1: 1\right) \text { gas } \\
\text { after complete dehydrogenation of formic acid with [Ru]-1. Reaction } \\
\text { condition: [Ru]-1 }(0.01 \mathrm{mmol}) \text {, formic acid }(0.4 \mathrm{M}, 2.5 \mathrm{~mL}) \text {, sodium } \\
\text { formate }(0.05 \mathrm{mmol}), 90^{\circ} \mathrm{C} \text {. (Analysis is performed using Argon as the } \\
\text { carrier gas). }\end{array}$ & S17 \\
\hline $\begin{array}{l}\text { Figure S15.(a) Temperature dependent formic acid dehydrogenation } \\
\text { over [Ru]-1, and the corresponding (b) Arrhenius plot of the initial TOF } \\
\text { values for formic acid }(2 \mathrm{M}, 2.5 \mathrm{~mL}) \text { dehydrogenation over }[\mathrm{Ru}]-1(0.2 \\
\text { mol\%), }\left[\mathrm{HCOONa} /[\mathrm{HCOOH}]=2: 1,60^{\circ} \mathrm{C}-90^{\circ} \mathrm{C} \text {. }\right.\end{array}$ & S18 \\
\hline $\begin{array}{l}\text { Figure S16. Recycling experiment for the catalytic dehydrogenation of } \\
\text { formic acid over [Ru]-1. Reaction condition: formic acid }(4.0 \mathrm{M}, 2.5 \\
\mathrm{mL}),[\mathrm{Ru}]-1(0.1 \mathrm{~mol} \%) \text {, [HCOONa]/[HCOOH] }=2: 1,90{ }^{\circ} \mathrm{C} .(10 \mathrm{mmol} \\
\text { of formic acid is added to the reaction mixture after each run). }\end{array}$ & S18 \\
\hline $\begin{array}{l}\text { Figure S17. Plot of } \ln \left[\text { initial rate }\left(\mathrm{mmolL}^{-1} \mathrm{~h}^{-1}\right)\right] \text { vs } \ln [\mathrm{cat}]\left(\mathrm{mmol} \mathrm{L}^{-1}\right) \text {. } \\
\text { Reaction condition: }[\mathrm{Ru}]-1(0.01 \mathrm{mmol}-0.04 \mathrm{mmol}) \text {, formic acid }(1.0 \\
\mathrm{M}, 2.5 \mathrm{~mL}), 90^{\circ} \mathrm{C} \text {. }\end{array}$ & S19 \\
\hline $\begin{array}{l}\left.\text { Figure S18. Plot of In[initial rate }\left(\mathrm{mmolL}^{-1} \mathrm{~h}^{-1}\right)\right] \text { vs } \ln [\mathrm{HCOOH}]\left(\mathrm{mmol} \mathrm{L}^{-1}\right) \text {. } \\
\text { Reaction condition: }[\mathrm{Ru}]-1(0.01 \mathrm{mmol}) \text {, formic acid }(0.2-0.8 \mathrm{M}, 2.5 \\
\mathrm{mL}), 90^{\circ} \mathrm{C} \text {. }\end{array}$ & S19 \\
\hline Figure S19. Transformation of colour of the reaction mixture during the & S20 \\
\hline
\end{tabular}


catalytic dehydrogenation of formic acid. Reaction Condition: [Ru]-1 $(0.01 \mathrm{mmol})$, formic acid $(0.4 \mathrm{M}, 2.5 \mathrm{~mL})$, sodium formate $(0.05 \mathrm{mmol})$, $\mathrm{T}=90^{\circ} \mathrm{C}$.

Figure S20. Mass spectra of the reaction aliquot for the base assisted catalytic dehydrogenation of formic acid after 5 minutes. Reaction condition: [Ru]-1 (0.01 mmol), formic acid $(0.4 \mathrm{M}, 2.5 \mathrm{~mL})$, sodium formate $(0.05 \mathrm{mmol}), \mathrm{T}=90^{\circ} \mathrm{C}$.

Figure S21. Mass spectra of the reaction aliquot at the end of the base assisted catalytic dehydrogenation of formic acid. Reaction condition: [Ru]-1 (0.01 mmol), formic acid $(0.4 \mathrm{M}, 2.5 \mathrm{~mL})$, sodium formate $(0.05$ $\mathrm{mmol}), \mathrm{T}=90^{\circ} \mathrm{C}$.

Figure S22. Mass investigation of the reaction mixture for catalytic dehydrogenation of formic acid over [Ru]-1 catalyst showing the colour change from the initial yellow to red-orange during the initial minutes and reverts back to yellow colour when treated with an excess of dil. $\mathrm{HCl}$.

Figure S23. Mass spectra of the reaction aliquot for the reaction of [Ru]-1 $(0.01 \mathrm{mmol})$ with formic acid $(0.4 \mathrm{M}, 2.5 \mathrm{~mL})$ added to it and stirred at room temperature for 10 minutes.

Figure S24. Observation of ruthenium-formate species [Ru]-1B (m/z $\left.412[\mathrm{M}]+\mathrm{Na}^{+}\right)$observed during mass investigation. Reaction condition: [Ru]-1 (0.01 mmol), formic acid (0.2 M, $2.5 \mathrm{~mL})$, sodium formate $(0.05$ $\mathrm{mmol}$ ) and stirred at room temperature for 10 minutes.

Figure S25. Mass spectra of the reaction aliquot for the reaction of [Ru]-1 $(0.01 \mathrm{mmol})$ with sodium formate $(0.04 \mathrm{M}, 2.5 \mathrm{~mL})$ at room temperature for 10 minutes.

Figure S26. Single crystal X-ray molecular structure of [Ru]-1A'.

Figure S27. Mass spectra of [Ru]-1A'

Figure S28. ${ }^{1} \mathrm{H}$ NMR spectra showing the generation of Ru-hydride species during the treatment of [Ru]-1 $(0.01 \mathrm{mmol})$ with sodium formate $(0.05 \mathrm{mmol})$ in $\mathrm{D}_{2} \mathrm{O}(0.6 \mathrm{~mL})$ at $25^{\circ} \mathrm{C}$

Table S6. Single crystal X-ray refinement data for [Ru]-1A'

Table S7. Selected bond lengths $(\AA)$ for complex [Ru]-1A'

Table S8. Selected bond angles $\left(^{\circ}\right)$ for complex [Ru]-1A'

Figure S29. Time-dependent GC-TCD analysis of the evolved gas $\left(\mathrm{H}_{2} \quad \mathrm{~S} 29\right.$ and $\mathrm{CO}_{2}$ ) during the catalytic formaldehyde dehydrogenation reaction. (a) initial 10 minutes; $\mathrm{H}_{2}: \mathrm{CO}_{2} \approx 6: 1$ (b) After 30 minutes; $\mathrm{H}_{2}: \mathrm{CO}_{2} \approx 2.2: 1$. (c) After completion of reaction; $\mathrm{H}_{2}: \mathrm{CO}_{2} \approx 2: 1$. Reaction condition: [Ru]$1(0.01 \mathrm{mmol})$, aq. formaldehyde $(0.4 \mathrm{M}, 2.5 \mathrm{~mL}), 90{ }^{\circ} \mathrm{C}$. (Analysis is performed using Argon as the carrier gas).

Figure S30. Time-dependent $\mathrm{pH}$ plot for the catalytic formaldehyde dehydrogenation reaction. Reaction condition: [Ru]-1 (0.01 mmol), formaldehyde $(0.4 \mathrm{M}, 2.5 \mathrm{~mL}), 90^{\circ} \mathrm{C}$. 


\begin{tabular}{|c|c|}
\hline $\begin{array}{l}\text { Figure S31. Transformation of colour of the reaction mixture during the } \\
\text { catalytic dehydrogenation of aqueous formaldehyde. Reaction } \\
\text { condition: }\left[R \mathbf{R u}-1(0.01 \mathrm{mmol}) \text {, aq. formaldehyde }(0.4 \mathrm{M}, 2.5 \mathrm{~mL}), 90^{\circ} \mathrm{C} \text {. }\right.\end{array}$ & S31 \\
\hline $\begin{array}{l}\text { Figure S32. Mass spectra of the reaction aliquot the catalytic } \\
\text { dehydrogenation of aq. formaldehyde after } 5 \text { minutes. Reaction } \\
\text { condition: [Ru]-1 }(0.01 \mathrm{mmol}) \text {, formaldehyde }(0.4 \mathrm{M}, 2.5 \mathrm{~mL}), \mathrm{T}=90 \\
{ }^{\circ} \mathrm{C} \text {. }\end{array}$ & S31 \\
\hline $\begin{array}{l}\text { Figure S33. Mass spectra of the reaction aliquot the catalytic } \\
\text { dehydrogenation of aq. formaldehyde after } 10 \text { minutes. Reaction } \\
\text { condition: [Ru]-1 }(0.01 \mathrm{mmol}) \text {, formaldehyde }(0.4 \mathrm{M}, 2.5 \mathrm{~mL}), \mathrm{T}=90 \\
{ }^{\circ} \mathrm{C} \text {. }\end{array}$ & S32 \\
\hline $\begin{array}{l}\text { Figure S34. Mass spectra of the reaction aliquot the catalytic } \\
\text { dehydrogenation of aq. formaldehyde after } 20 \text { minutes. Reaction } \\
\text { condition: [Ru]-1 }(0.01 \mathrm{mmol}) \text {, formaldehyde }(0.4 \mathrm{M}, 2.5 \mathrm{~mL}), \mathrm{T}=90 \\
{ }^{\circ} \mathrm{C} \text {. }\end{array}$ & S32 \\
\hline $\begin{array}{l}\text { Figure S35. Time course plot for the catalytic dehydrogenation of } \\
\text { aqueous formaldehyde }(13.55 \mathrm{mmol}) \text { over the catalyst [Ru]-1(0.1 mol } \\
\%), \mathrm{T}=95^{\circ} \mathrm{C} \text {. }\end{array}$ & S33 \\
\hline${ }^{1} \mathrm{H},{ }^{13} \mathrm{C}$ NMR and mass spectra of the ligands and complexes & S34-S41 \\
\hline References & S41-S43 \\
\hline
\end{tabular}

\section{Conversion, TON and TOF}

The conversion, TON and TOF are calculated as per earlier reported procedures. ${ }^{\text {S1 }}$ Typically for formic acid dehydrogenation, TON is calculated by the formula [(substrate/catalyst) $\times$ (conversion/100)], whereas for formaldehyde dehydrogenation reaction, TON is calculated by the formula $\left[n\left(\mathrm{H}_{2}+\mathrm{CO}_{2}\right) / n\right.$ (catalyst)]. The turnover frequency (TOF) is calculated as TON/time. 
Table S1. Comparative catalytic activity of previous literature work and the current work for formic acid dehydrogenation.

\begin{tabular}{|c|c|c|c|c|c|c|c|}
\hline Catalyst & Substrate & solvent & $\begin{array}{c}\mathbf{T} \\
\left({ }^{\circ} \mathrm{C}\right)\end{array}$ & $\mathbf{t}(\mathrm{h})$ & TON & TOF $\left(\mathrm{h}^{-1}\right)$ & Ref. \\
\hline $7^{+}$ & $\begin{array}{l}\mathrm{HCOOH} / \\
\mathrm{HCOONa}\end{array}$ & $\mathrm{H}_{2} \mathrm{O}$ & 90 & 0.25 & 6050 & 1548 & $\begin{array}{l}\text { This } \\
\text { work }\end{array}$ \\
\hline & $\begin{array}{l}\mathrm{HCOOH} / \\
\mathrm{HCOONa}\end{array}$ & $\mathrm{H}_{2} \mathrm{O}$ & 90 & 0.25 & 2248 & 940 & S1 \\
\hline$\left[(\mathrm{Cp} * \mathrm{Ir})_{2}(\mathrm{THBPM}) \mathrm{Cl}_{2}\right] \mathrm{Cl}_{2}$ & $\begin{array}{l}\mathrm{HCOOH} / \\
\mathrm{HCOONa}\end{array}$ & $\mathrm{H}_{2} \mathrm{O}$ & 90 & 0.25 & 165000 & 228000 & S2 \\
\hline $\begin{array}{l}{\left[\mathrm{Cp} * \operatorname{Ir}\left(4,4^{\prime}-\right.\right.} \\
\left.\operatorname{DHBP})\left(\mathrm{H}_{2} \mathrm{O}\right)\right] \mathrm{SO}_{4}\end{array}$ & $\mathrm{HCOOH}$ & $\mathrm{H}_{2} \mathrm{O}$ & 90 & NA & NA & 14000 & S3 \\
\hline$\left[\mathrm{Cp} * \operatorname{Ir}(\mathrm{TMBI}) \mathrm{H}_{2} \mathrm{O}\right] \mathrm{SO}_{4}$ & $\mathrm{HCOOH}$ & $\mathrm{H}_{2} \mathrm{O}$ & 80 & 0.17 & 10000 & 34000 & S4 \\
\hline $\begin{array}{l}{[\mathrm{Cp} * \mathrm{Ir} \text { (pyrimidylimidazoline }} \\
\left.\mathrm{H}_{2} \mathrm{O}\right] \mathrm{SO}_{4}\end{array}$ & $\begin{array}{l}\mathrm{HCOOH} / \\
\mathrm{HCOONa}\end{array}$ & $\mathrm{H}_{2} \mathrm{O}$ & 100 & 0.17 & 68000 & 322000 & S5 \\
\hline$\left[\mathrm{Cp}^{*} \operatorname{Ir}(\mathrm{THBPM})\left(\mathrm{H}_{2} \mathrm{O}\right)\right] \mathrm{SO}_{4}$ & $\begin{array}{l}\mathrm{HCOOH} / \\
\mathrm{HCOONa}\end{array}$ & $\mathrm{H}_{2} \mathrm{O}$ & 80 & 0.08 & 11000 & 39500 & S6 \\
\hline$\left[\mathrm{Cp} * \operatorname{Ir}(\mathrm{PHEN}-\mathrm{diol}) \mathrm{H}_{2} \mathrm{O}\right] \mathrm{SO}_{4}$ & $\mathrm{HCOOH}$ & $\mathrm{H}_{2} \mathrm{O}$ & 60 & 2600 & 5000000 & 1900 & S7 \\
\hline$-\mathrm{NH} \stackrel{\mathrm{H}}{\mathrm{N}}+\mathrm{Cl}^{-}$ & $\mathrm{HCOOH}$ & $\mathrm{H}_{2} \mathrm{O}$ & 90 & 0.005 & 47000 & 487500 & S8 \\
\hline$\sqrt{-7}+\mathrm{Cl}^{-}$ & $\mathrm{HCOOH}$ & $\mathrm{H}_{2} \mathrm{O}$ & 80 & 14 & 2400000 & 171400 & S8 \\
\hline
\end{tabular}







Table S2. Single crystal X-ray refinement data for complex [Ru]-2

\begin{tabular}{|c|c|}
\hline \multicolumn{2}{|c|}{$[\mathrm{Ru}]-2$} \\
\hline Formula & $\mathrm{C}_{12} \mathrm{H}_{17} \mathrm{Cl}_{2} \mathrm{NO}_{3} \mathrm{Ru}$ \\
\hline Molecular weight & 395.23 \\
\hline Crystal system & Triclinic \\
\hline Space group & P-1 \\
\hline Temperature/K & 293 \\
\hline Wavelength & 0.71073 \\
\hline$a / \AA$ & $6.7939(3)$ \\
\hline$b / \AA$ & $9.0656(5)$ \\
\hline$c / \AA$ & $13.3758(7)$ \\
\hline$\alpha /^{\circ}$ & $98.946(5)$ \\
\hline$\beta /^{\circ}$ & $103.638(4)$ \\
\hline$\gamma /{ }^{\circ}$ & $105.280(4)$ \\
\hline $\mathrm{V} / \AA^{3}$ & 751.19 \\
\hline Z & 2 \\
\hline Density $/ \mathrm{gcm}^{-1}$ & 1.747 \\
\hline Absorption Coefficient & 1.401 \\
\hline Absorption Correction & $\begin{array}{l}\text { Semi empirical from } \\
\text { equivalents }\end{array}$ \\
\hline $\mathrm{F}(000)$ & 396.0 \\
\hline Total no of reflections & 5945 \\
\hline Reflections, $I>2 \sigma(I)$ & 3396 \\
\hline Max. $2 \theta /^{\circ}$ & 28.807 \\
\hline Ranges (h, k, l) & $\begin{array}{l}-9 \leq \mathrm{h} \leq 8 \\
-9 \leq \mathrm{k} \leq 12 \\
-16 \leq 1 \leq 16\end{array}$ \\
\hline Complete to $2 \theta(\%)$ & $99 . \overline{9}$ \\
\hline Refinement method & $\begin{array}{l}\text { Full-matrix least- } \\
\text { squares on } F^{2}\end{array}$ \\
\hline Goof $\left(F^{2}\right)$ & 1.027 \\
\hline $\mathrm{R}$ indices $[I>2 \sigma(I)]$ & 0.0238 \\
\hline R Indices (all data) & 0.0250 \\
\hline
\end{tabular}


Table S3. Selected bond lengths ( $\AA$ ) for complex [Ru]-2

\begin{tabular}{ll}
\hline $\mathrm{Ru}(1)-\mathrm{N}(1)$ & $2.1035(16)$ \\
$\mathrm{Ru}(1)-\mathrm{O}(1)$ & $2.1307(14)$ \\
$\mathrm{Ru}(1)-\mathrm{C}(11)$ & $2.153(2)$ \\
$\mathrm{Ru}(1)-\mathrm{C}(12)$ & $2.159(2)$ \\
$\mathrm{Ru}(1)-\mathrm{C}(7)$ & $2.177(2)$ \\
$\mathrm{Ru}(1)-\mathrm{C}(10)$ & $2.177(2)$ \\
$\mathrm{Ru}(1)-\mathrm{C}(8)$ & $2.179(2)$ \\
$\mathrm{Ru}(1)-\mathrm{C}(9)$ & $2.184(2)$ \\
$\mathrm{Ru}(1)-\mathrm{Cl}(1)$ & $2.4060(5)$ \\
$\mathrm{O}(1)-\mathrm{C}(6)$ & $1.434(3)$ \\
$\mathrm{N}(1)-\mathrm{C}(1)$ & $1.347(3)$ \\
$\mathrm{N}(1)-\mathrm{C}(5)$ & $1.351(3)$ \\
\hline
\end{tabular}

Table S4. Selected bond angles $\left({ }^{\circ}\right)$ for complex $[R u]-2$

\begin{tabular}{ll}
\hline $\mathrm{N}(1)-\mathrm{Ru}(1)-\mathrm{O}(1)$ & $76.08(6)$ \\
$\mathrm{N}(1)-\mathrm{Ru}(1)-\mathrm{C}(11)$ & $123.45(11)$ \\
$\mathrm{O}(1)-\mathrm{Ru}(1)-\mathrm{C}(11)$ & $159.86(11)$ \\
$\mathrm{N}(1)-\mathrm{Ru}(1)-\mathrm{C}(12)$ & $97.43(9)$ \\
$\mathrm{O}(1)-\mathrm{Ru}(1)-\mathrm{C}(12)$ & $155.02(9)$ \\
$\mathrm{C}(11)-\mathrm{Ru}(1)-\mathrm{C}(12)$ & $38.01(12)$ \\
$\mathrm{N}(1)-\mathrm{Ru}(1)-\mathrm{C}(7)$ & $96.14(8)$ \\
$\mathrm{O}(1)-\mathrm{Ru}(1)-\mathrm{C}(7)$ & $117.96(8)$ \\
$\mathrm{C}(11)-\mathrm{Ru}(1)-\mathrm{C}(7)$ & $68.36(10)$ \\
$\mathrm{C}(12)-\mathrm{Ru}(1)-\mathrm{C}(7)$ & $37.84(10)$ \\
$\mathrm{N}(1)-\mathrm{Ru}(1)-\mathrm{C}(10)$ & $161.61(11)$ \\
$\mathrm{O}(1)-\mathrm{Ru}(1)-\mathrm{C}(10)$ & $121.62(10)$ \\
$\mathrm{C}(11)-\mathrm{Ru}(1)-\mathrm{C}(10)$ & $38.48(12)$ \\
$\mathrm{C}(12)-\mathrm{Ru}(1)-\mathrm{C}(10)$ & $68.61(11)$ \\
$\mathrm{C}(7)-\mathrm{Ru}(1)-\mathrm{C}(10)$ & $80.24(9)$ \\
$\mathrm{N}(1)-\mathrm{Ru}(1)-\mathrm{C}(8)$ & $119.12(8)$ \\
$\mathrm{O}(1)-\mathrm{Ru}(1)-\mathrm{C}(8)$ & $94.12(7)$ \\
\hline
\end{tabular}




\begin{tabular}{ll}
\hline $\mathrm{C}(11)-\mathrm{Ru}(1)-\mathrm{C}(8)$ & $80.74(9)$ \\
$\mathrm{C}(12)-\mathrm{Ru}(1)-\mathrm{C}(8)$ & $67.62(9)$ \\
$\mathrm{C}(7)-\mathrm{Ru}(1)-\mathrm{C}(8)$ & $36.74(9)$ \\
$\mathrm{C}(10)-\mathrm{Ru}(1)-\mathrm{C}(8)$ & $67.84(10)$ \\
$\mathrm{N}(1)-\mathrm{Ru}(1)-\mathrm{C}(9)$ & $155.81(9)$ \\
$\mathrm{O}(1)-\mathrm{Ru}(1)-\mathrm{C}(9)$ & $95.90(8)$ \\
$\mathrm{C}(11)-\mathrm{Ru}(1)-\mathrm{C}(9)$ & $68.15(10)$ \\
$\mathrm{C}(12)-\mathrm{Ru}(1)-\mathrm{C}(9)$ & $80.14(10)$ \\
$\mathrm{C}(7)-\mathrm{Ru}(1)-\mathrm{C}(9)$ & $67.12(9)$ \\
$\mathrm{C}(10)-\mathrm{Ru}(1)-\mathrm{C}(9)$ & $37.12(10)$ \\
$\mathrm{C}(8)-\mathrm{Ru}(1)-\mathrm{C}(9)$ & $37.70(9)$ \\
$\mathrm{N}(1)-\mathrm{Ru}(1)-\mathrm{Cl}(1)$ & $84.07(5)$ \\
$\mathrm{O}(1)-\mathrm{Ru}(1)-\mathrm{Cl}(1)$ & $85.76(4)$ \\
$\mathrm{C}(11)-\mathrm{Ru}(1)-\mathrm{Cl}(1)$ & $91.19(7)$ \\
$\mathrm{C}(12)-\mathrm{Ru}(1)-\mathrm{Cl}(1)$ & $117.94(8)$ \\
$\mathrm{C}(7)-\mathrm{Ru}(1)-\mathrm{Cl}(1)$ & $155.72(7)$ \\
$\mathrm{C}(10)-\mathrm{Ru}(1)-\mathrm{Cl}(1)$ & $91.91(7)$ \\
$\mathrm{C}(8)-\mathrm{Ru}(1)-\mathrm{Cl}(1)$ & $156.14(7)$ \\
$\mathrm{C}(9)-\mathrm{Ru}(1)-\mathrm{Cl}(1)$ & $118.52(7)$ \\
\hline
\end{tabular}

Table S5. Optimization of reaction condition for the catalytic dehydrogenation of formic acid over [Ru]-1 in water at $90^{\circ} \mathrm{C}^{a}$

$\begin{array}{ccccccc}\text { entry } & \text { catalyst } & \begin{array}{c}\mathrm{HCOOH} \\ (\mathrm{mmol})\end{array} & \begin{array}{c}\mathrm{HCOONa} \\ (\mathrm{mmol})\end{array} & \begin{array}{c}\text { conv. }(\%)^{b} \\ \mathrm{TON}^{b}\end{array} & \begin{array}{c}\text { initial } \\ \left.\mathrm{TOF}^{-1}\right)\end{array} \\ 1 & {[\mathrm{Ru}]-1} & 1 & - & 95 & 95 & 146 \\ 2 & {[\mathrm{Ru}]-1} & - & 1 & - & - & - \\ 3 & {[\mathrm{Ru}]-1} & 1 & 0.02 & 95 & 95 & 228 \\ 4 & {[\mathrm{Ru}]-1} & 1 & 0.04 & 98 & 98 & 490 \\ 5 & {[\mathrm{Ru}]-1} & 1 & 0.05 & 98 & 98 & 660 \\ 6 & {[\mathrm{Ru}]-1} & 1 & 0.5 & 95 & 95 & 1141 \\ 7 & {[\mathrm{Ru}]-1} & 5 & 5 & 90 & 450 & 1241 \\ 8 & {[\mathrm{Ru}]-1} & 5 & 10 & 90 & 450 & 1446 \\ 9 & {[\mathrm{Ru}]-1} & 5 & 15 & 90 & 450 & 1262 \\ 10 & {[\mathrm{Ru}]-1} & 10 & 20 & 88 & 880 & 1548\end{array}$

${ }^{a}$ [Ru]-1 (0.01 mmol). Reactions are performed with an initial volume $2.5 \mathrm{~mL}$. Each reaction is repeated twice. ${ }^{b} \mathrm{Conv} .(\%)$ and TON are at the completion of the reaction. 


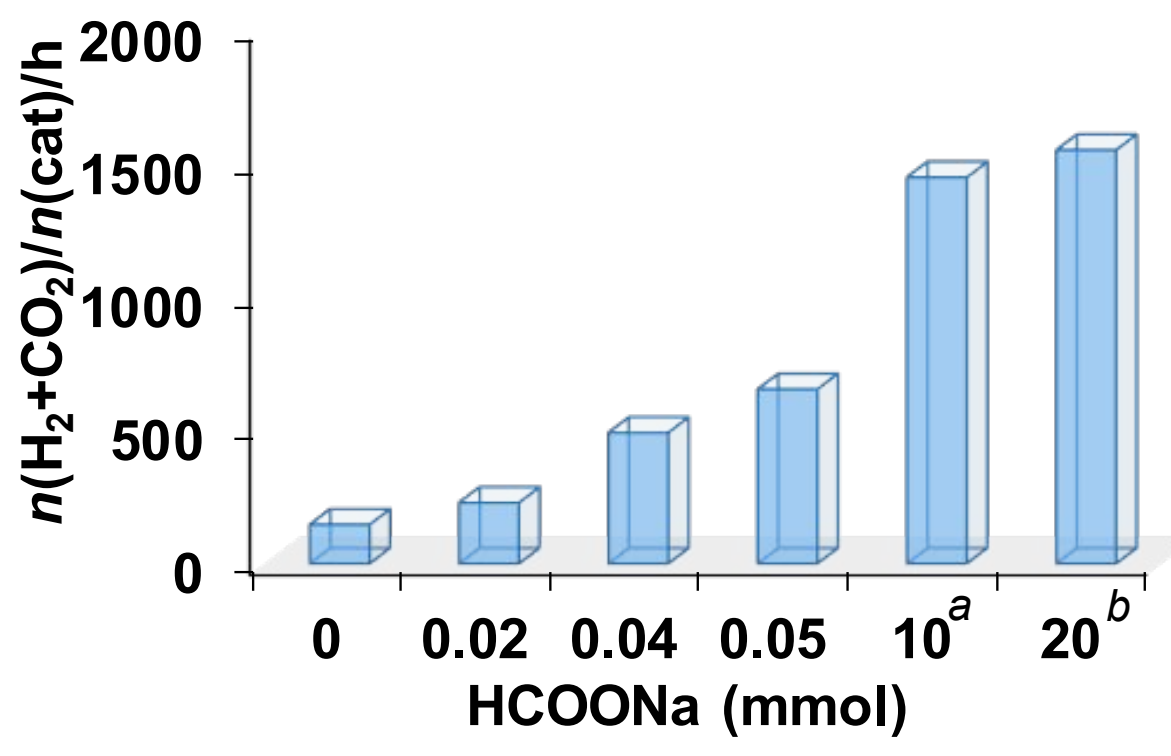

Figure S1.TOF $\left(\mathrm{h}^{-1}\right)$ vs [HCOONa] (mmol) plot for the catalytic dehydrogenation of formic acid over [Ru]-1. Reaction condition: formic acid (0.4 M, $2.5 \mathrm{~mL})$, [Ru]-1 (0.01 $\mathrm{mmol}), 90^{\circ} \mathrm{C}$. ${ }^{\text {aformic }}$ acid $(2.0 \mathrm{M}, 2.5 \mathrm{~mL}) .{ }^{b}$ formic acid $(4.0 \mathrm{M}, 2.5 \mathrm{~mL})$.

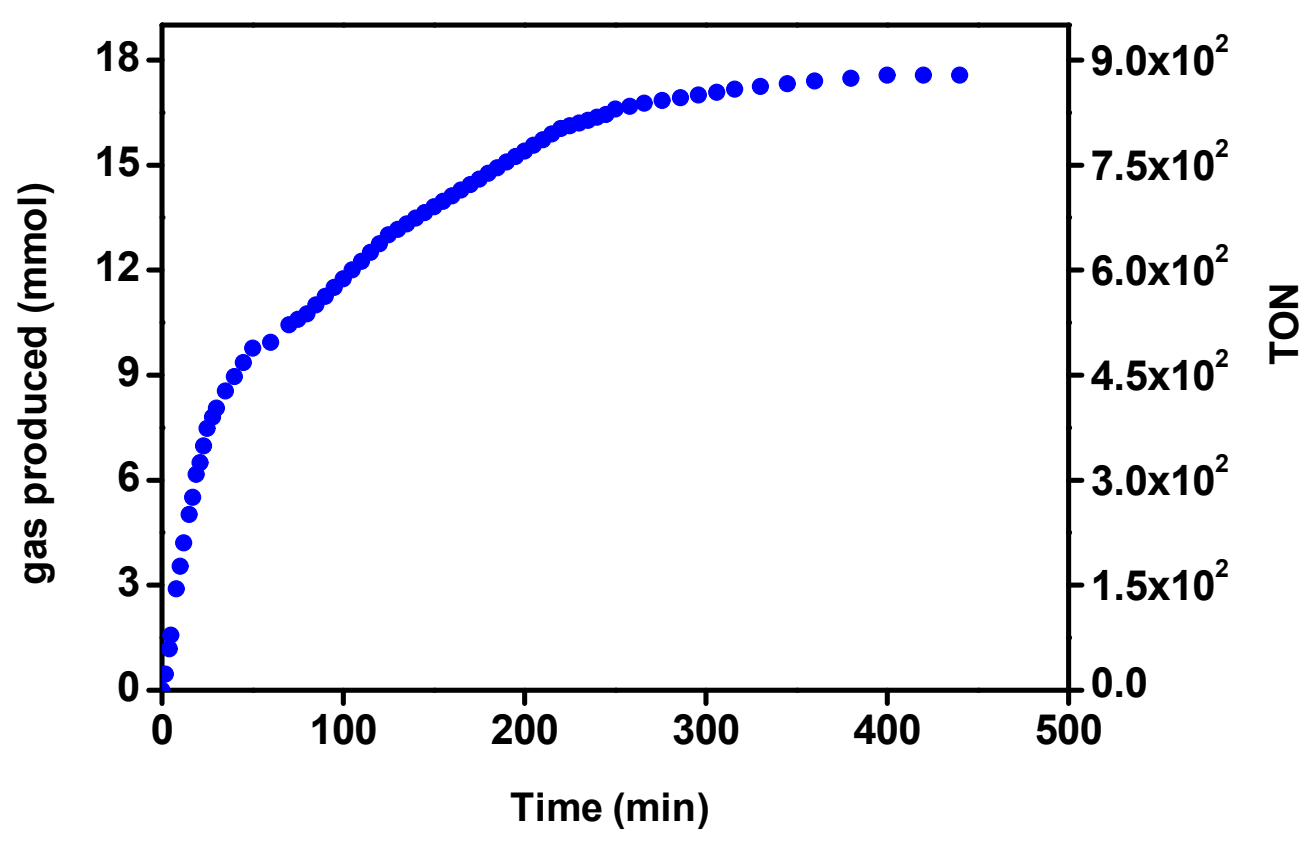

Figure S2. Gas produced (mmol) vs time ( $\mathrm{min})$ plot for the catalytic dehydrogenation of formic acid over [Ru]-1. Reaction condition: formic acid (4.0 M, $2.5 \mathrm{~mL})$, $[\mathrm{HCOONa}] /[\mathrm{HCOOH}]=2: 1,[\mathrm{Ru}]-1(0.01 \mathrm{mmol}), 90^{\circ} \mathrm{C}$. 


\section{Intensity}

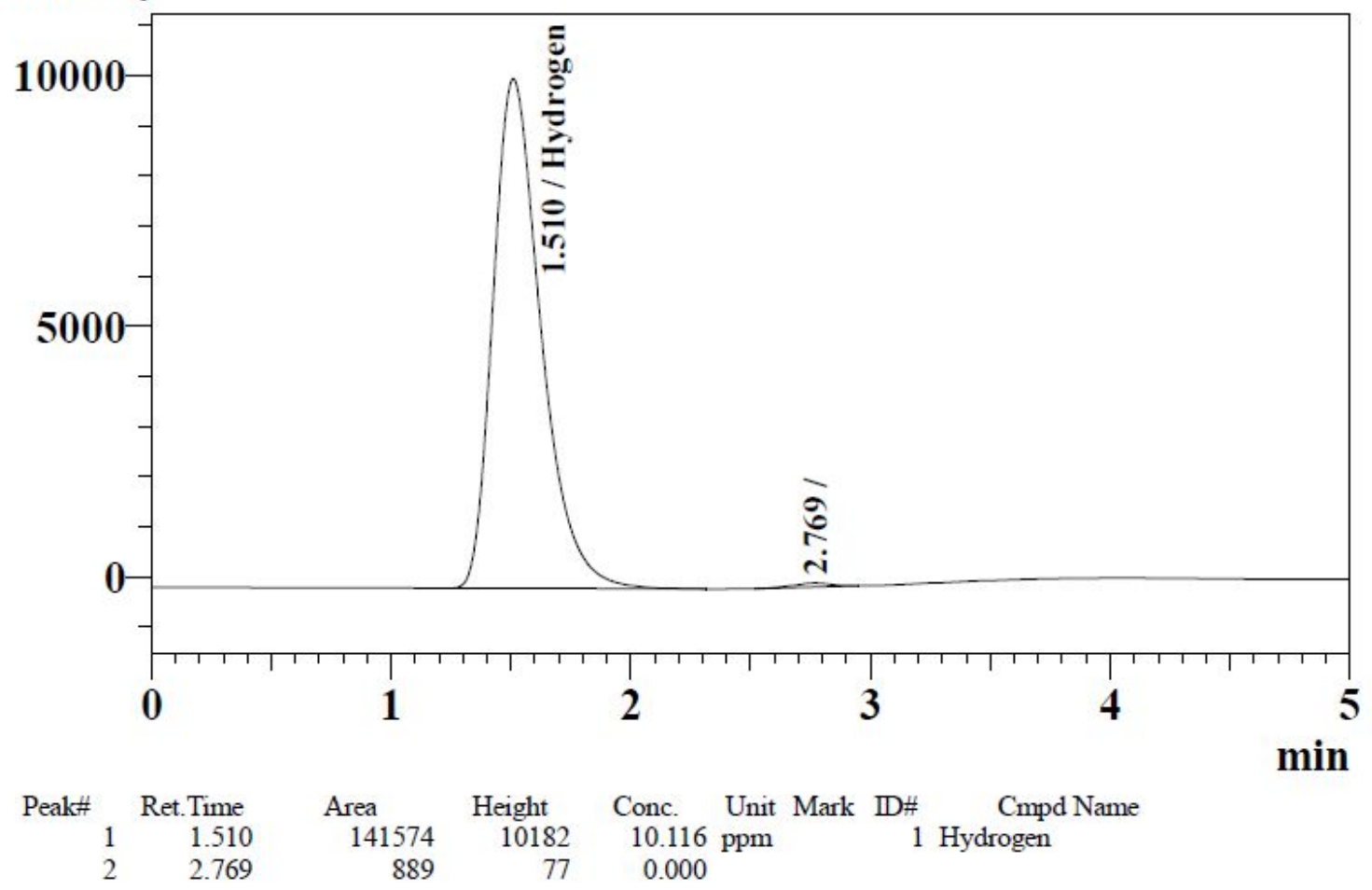

Figure S3. GC-TCD scan of $10 \mu \mathrm{L}$ pure $\mathrm{H}_{2}$ gas injected with a gas tight Hamilton syringe.

\section{Intensity}

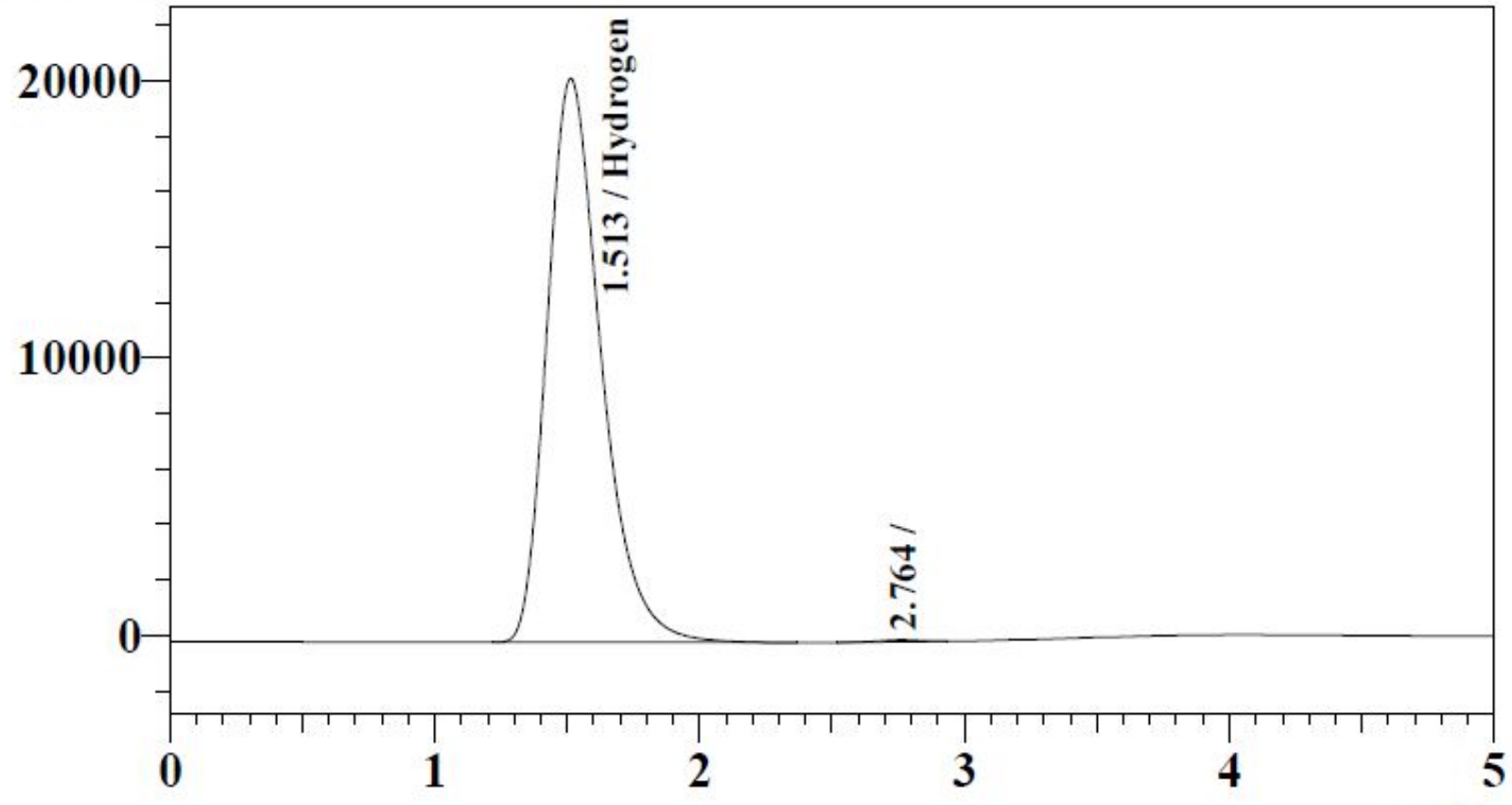

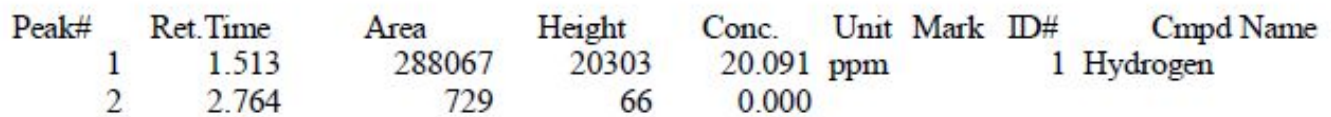

Figure S4. GC-TCD scan of $20 \mu \mathrm{L}$ pure $\mathrm{H}_{2}$ gas injected with a gas tight Hamilton syringe. 


\section{Intensity}

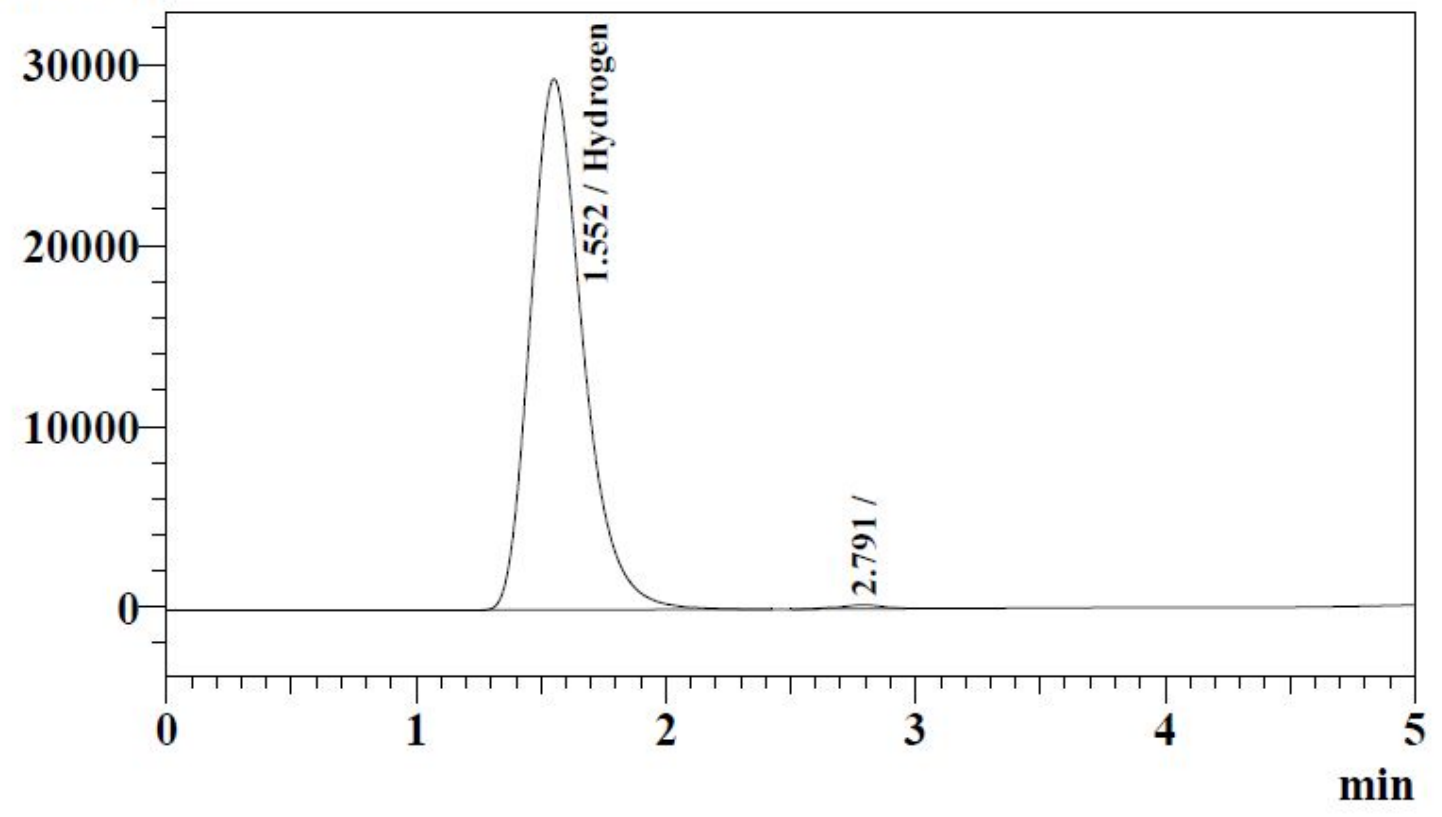

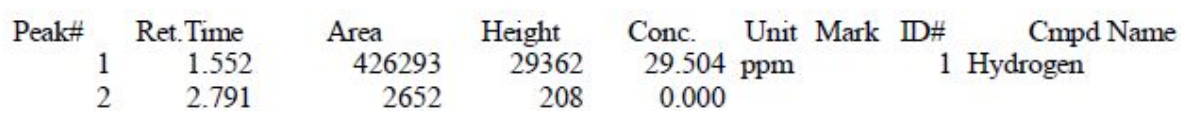

Figure S5. GC-TCD scan of $30 \mu \mathrm{L}$ pure $\mathrm{H}_{2}$ gas injected with a gas tight Hamilton syringe.

\section{Intensity}

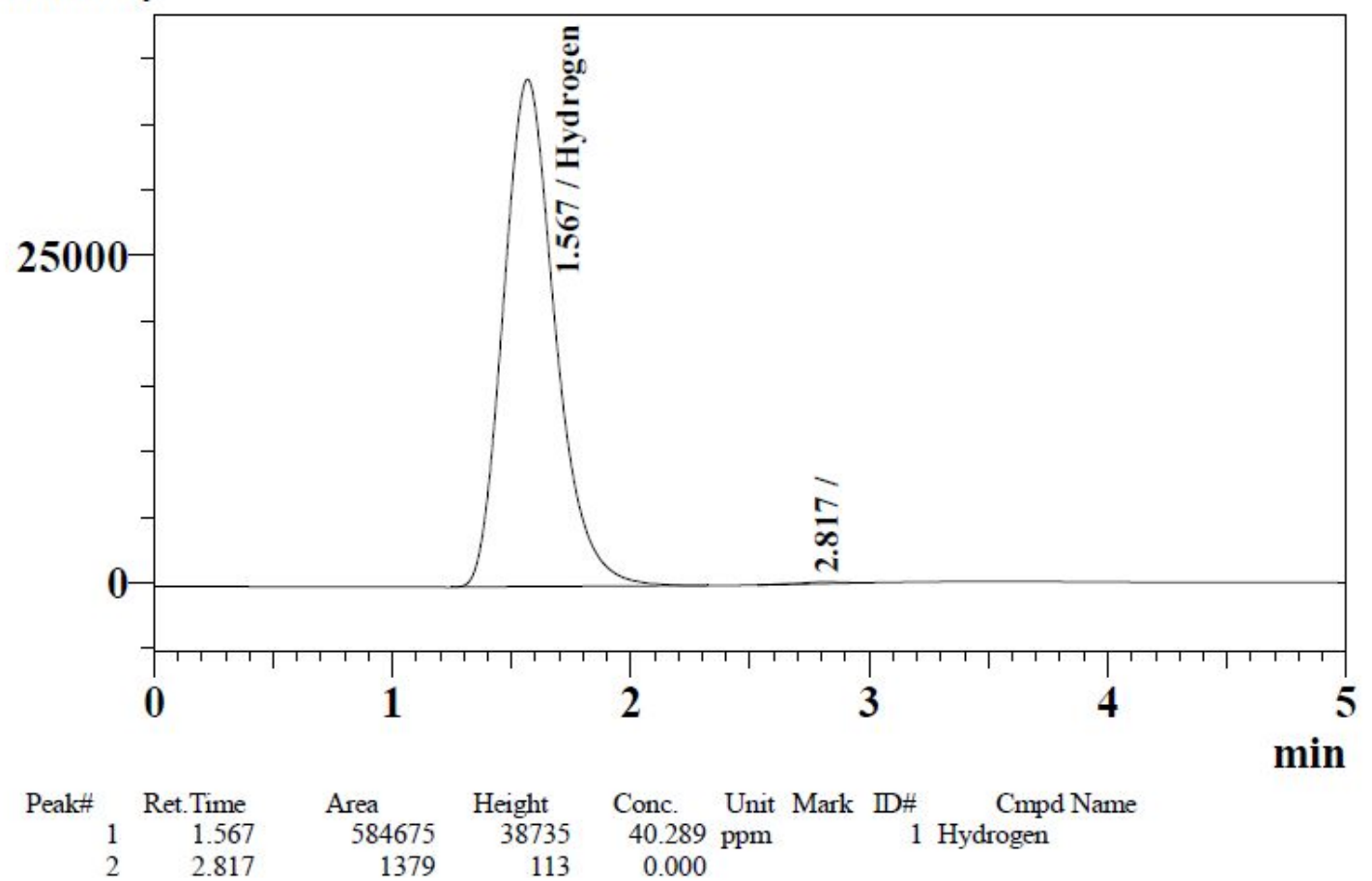

Figure S6. GC-TCD scan of $40 \mu \mathrm{L}$ pure $\mathrm{H}_{2}$ gas injected with a gas tight Hamilton syringe. 
ID\#:1 Name:Hydrogen

Calibration Curve - Analytical Line 1 - Channel 1

$f(x)=6.80939434721 e-005^{*} x+0.475810979358$

$\mathrm{R}=0.999648575259 \quad \mathrm{R}^{\wedge} 2=0.999297274018$

MeanRF:6.97127061444e-005 RFSD:1.00896499737e-006 RFRSD:1.44731864988

CurveType:Linear

ZeroThrough:Not through

WeightedRegression:None

External Standard

$\left[{ }^{*} 10^{\wedge} 1\right]$

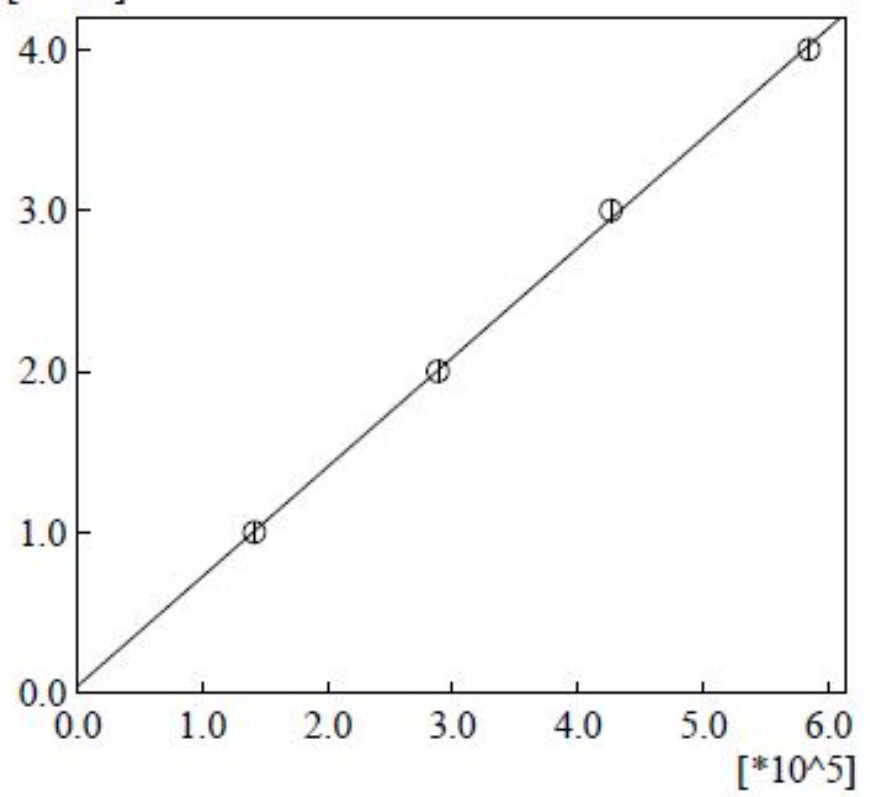

No. Conc. Area

$1 \quad 10.000$

20.000

$3 \quad 30.000$

40.000

426293

584675

Figure S7. Calibration plot of $\mathrm{H}_{2}$ gas. 


\section{Intensity}

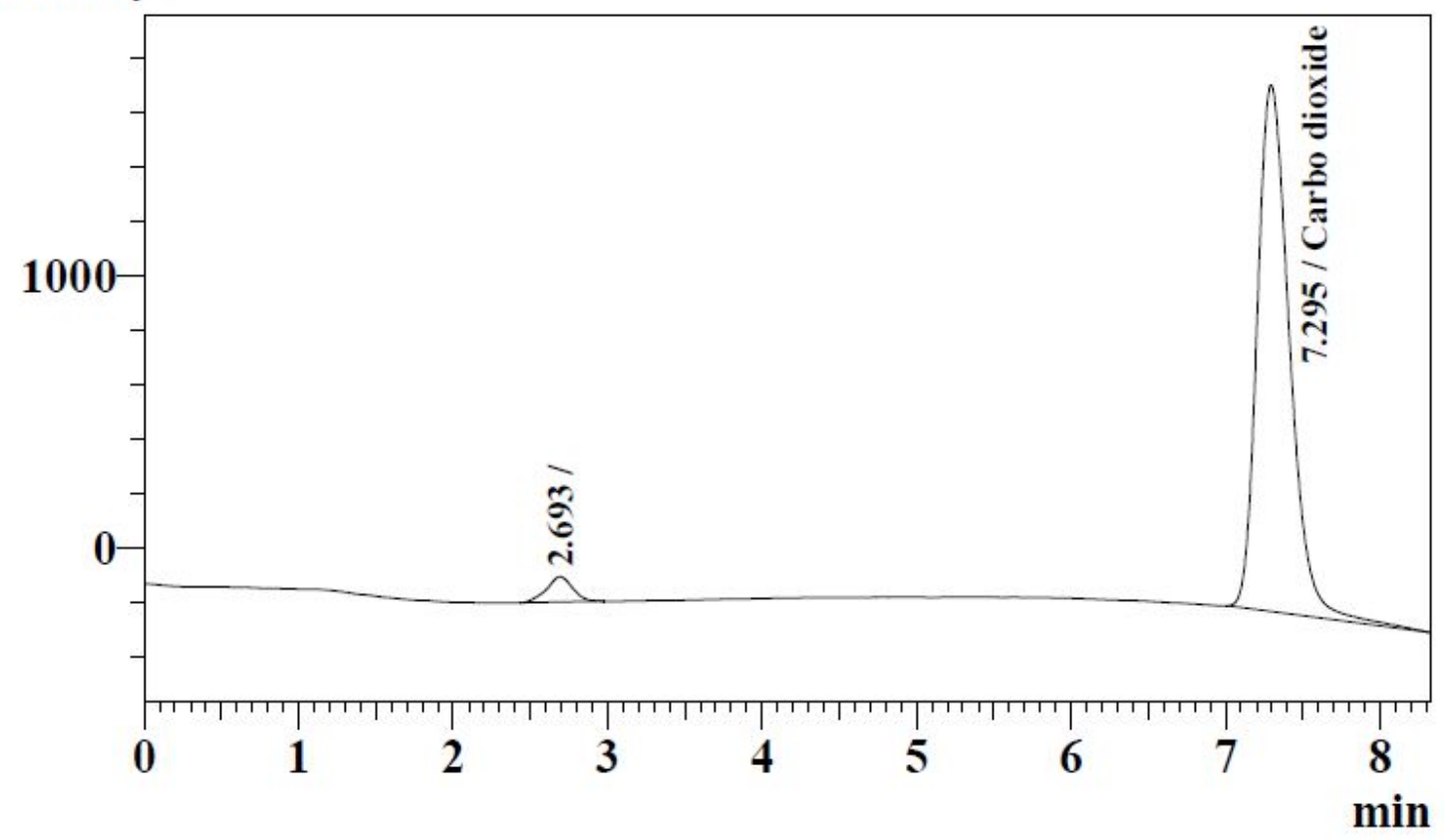

Figure S8. GC-TCD scan of $20 \mu \mathrm{L}$ pure $\mathrm{CO}_{2}$ gas injected with a gas tight Hamilton syringe.

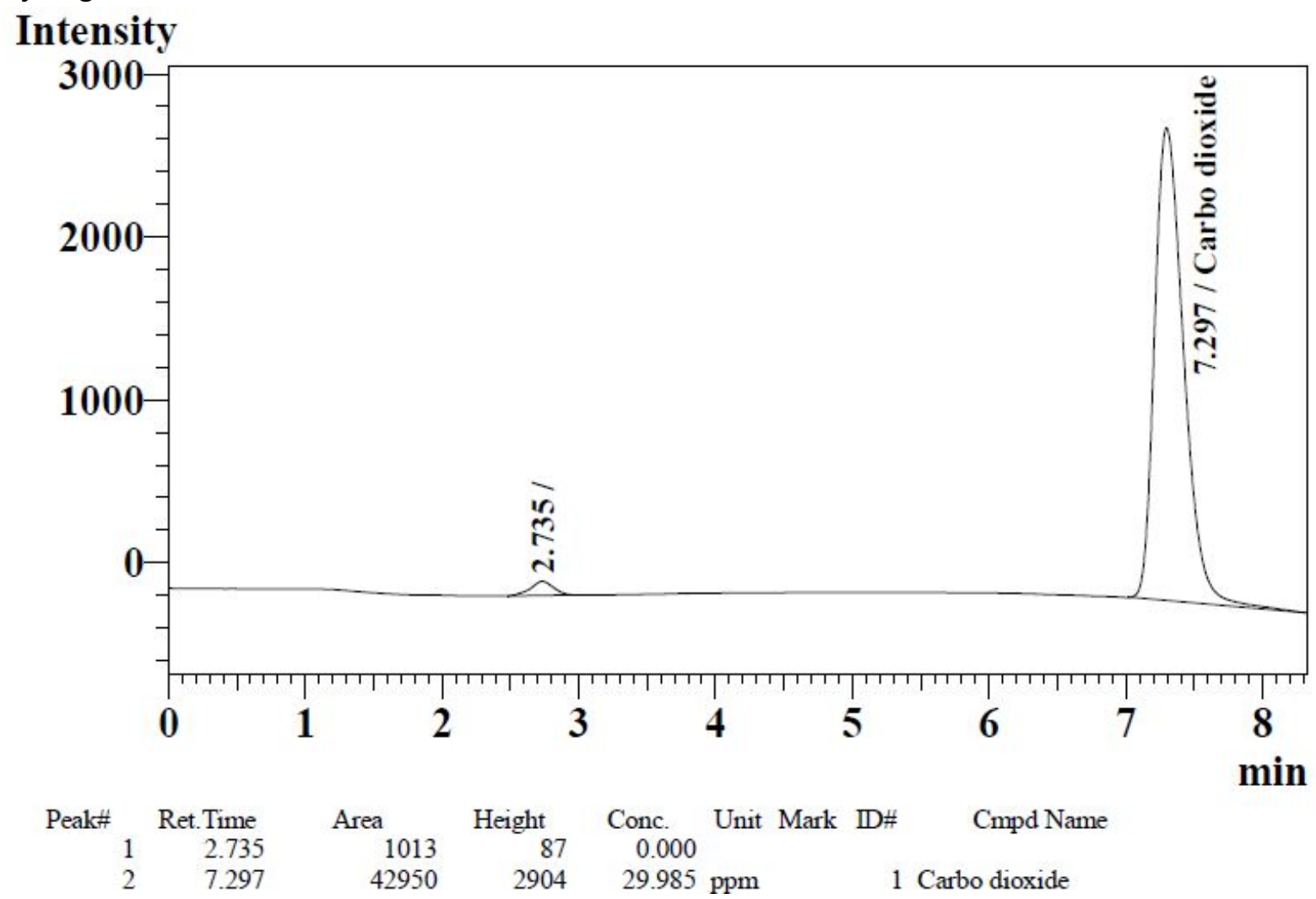

Figure S9. GC-TCD scan of $30 \mu \mathrm{L}$ pure $\mathrm{CO}_{2}$ gas injected with a gas tight Hamilton syringe. 


\section{Intensity}

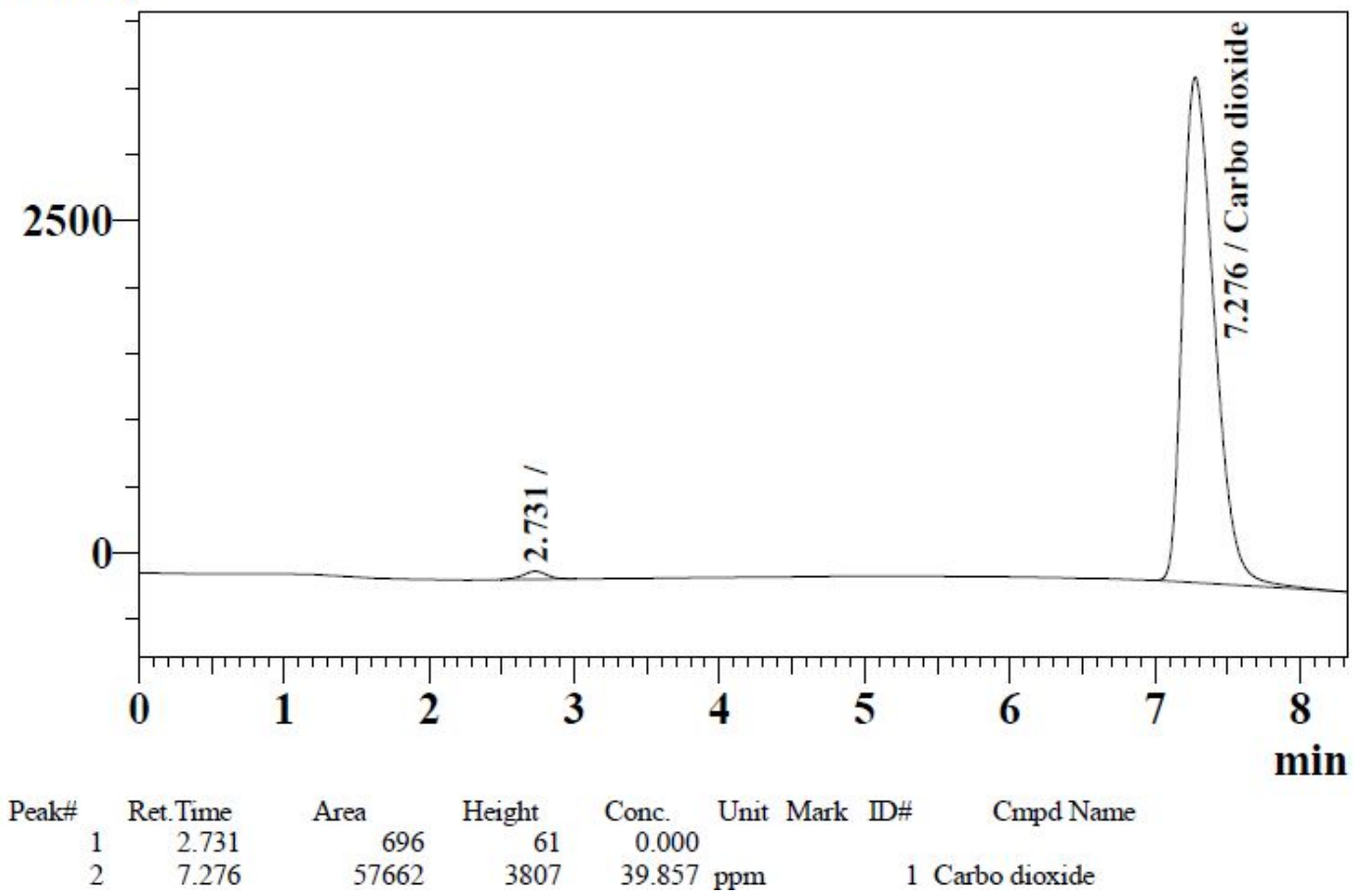

Figure S10. GC-TCD scan of $40 \mu \mathrm{L}$ pure $\mathrm{CO}_{2}$ gas injected with a gas tight Hamilton syringe.

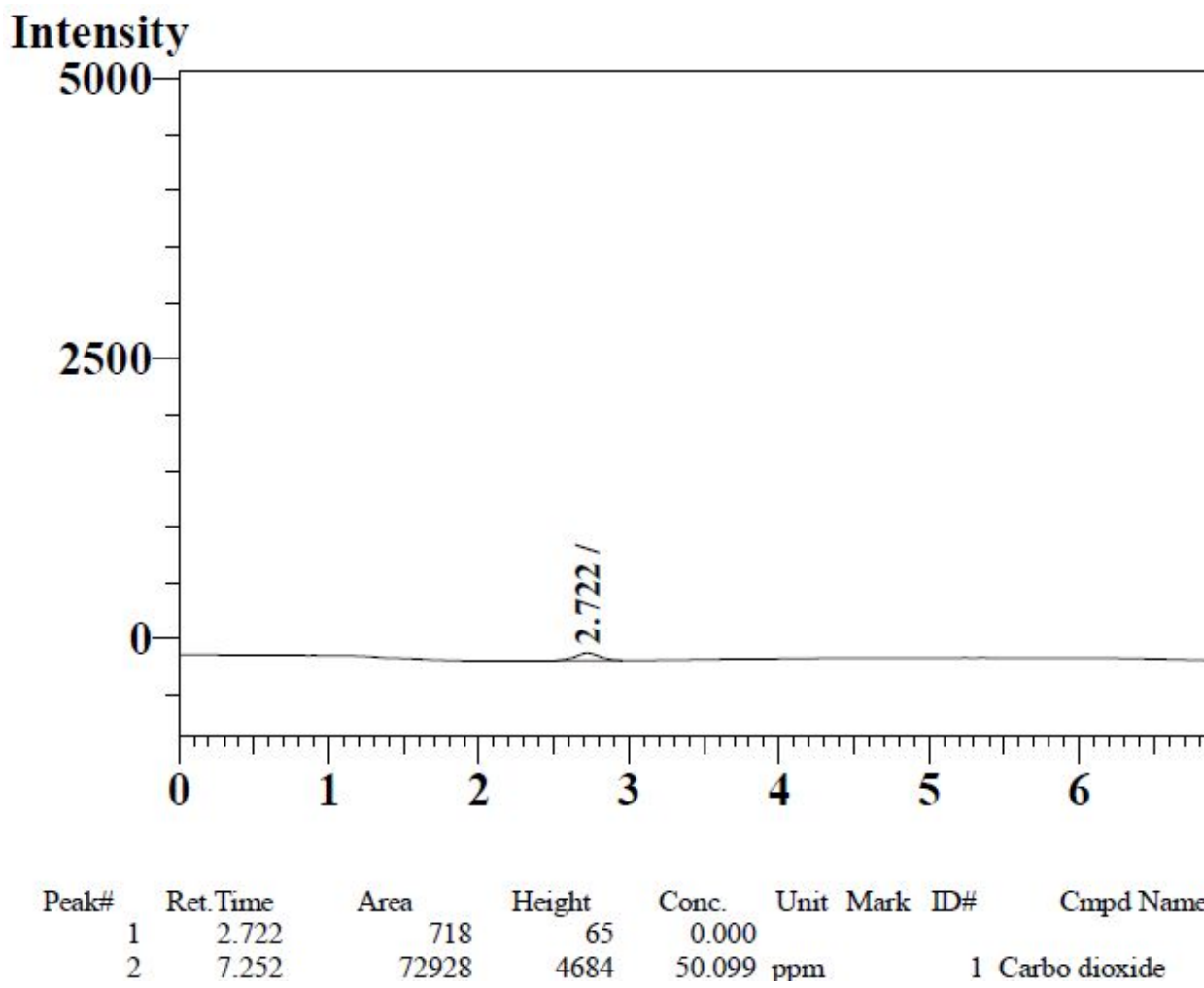

Figure S11. GC-TCD scan of $50 \mu \mathrm{L}$ pure $\mathrm{CO}_{2}$ gas injected with a gas tight Hamilton syringe. 
ID\#:1 Name:Carbo dioxide

$f(x)=6.70960953583 e-004 * x+1.1677206795$

$\mathrm{R}=0.999965944148 \quad \mathrm{R}^{\wedge} 2=0.999931889456$

MeanRF:6.97037759164e-004 RFSD:1.03459447245e-005 RFRSD:1.48427321024

CurveType:Linear

ZeroThrough:Not through

WeightedRegression:None

External Standard

$\left[{ }^{*} 10^{\wedge} 1\right]$

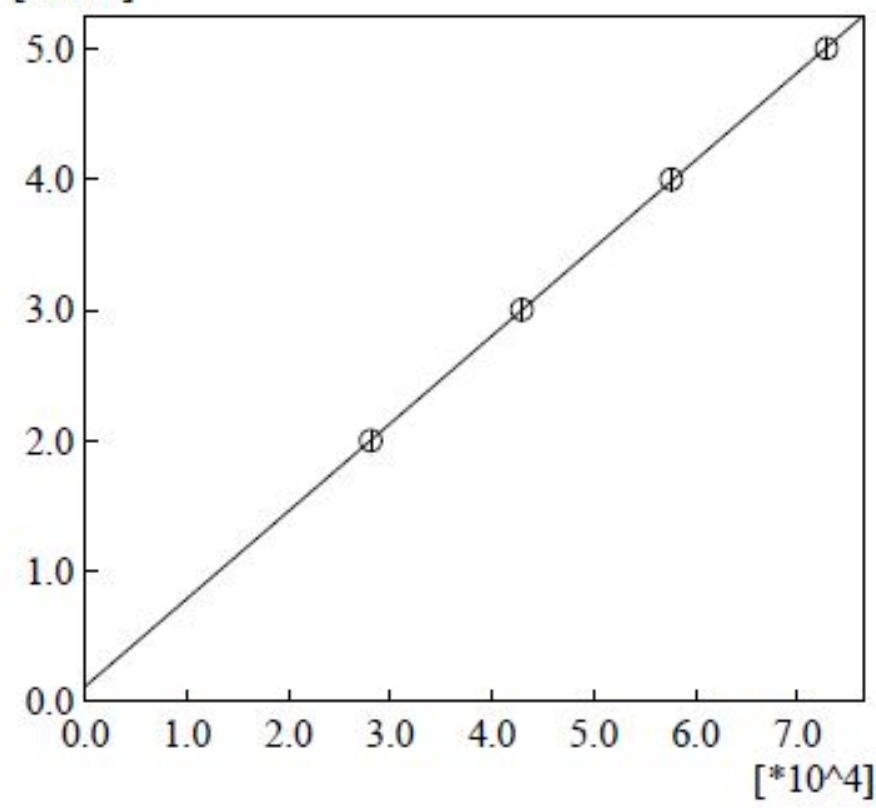

No. Conc.

20.000

Area

30.000

40.000

50.000

28155

42950

57662

4

72928

Figure S12. Calibration plot of $\mathrm{CO}_{2}$ gas. 


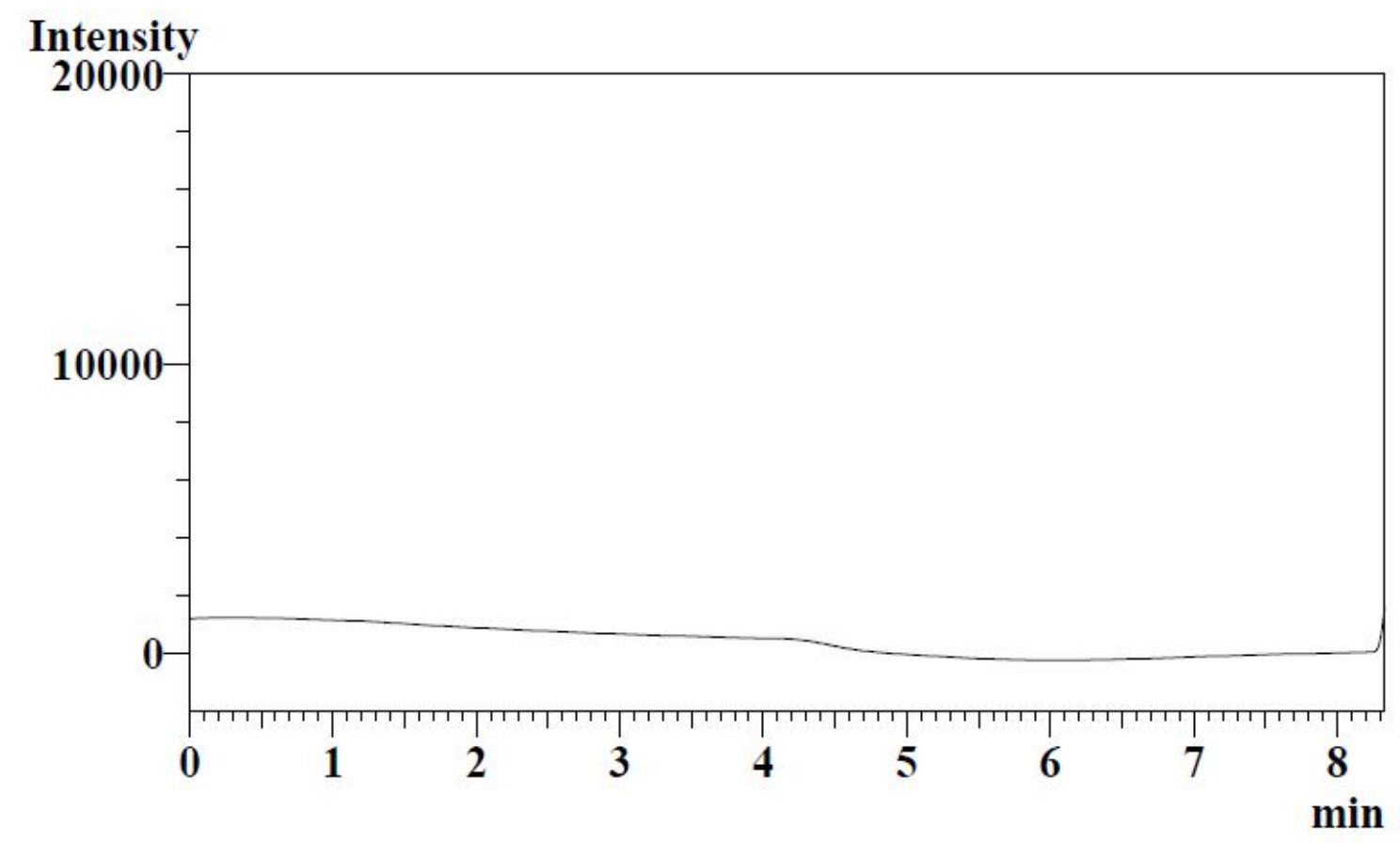

Figure S13. GC-TCD scan of blank sample using argon as the carrier gas

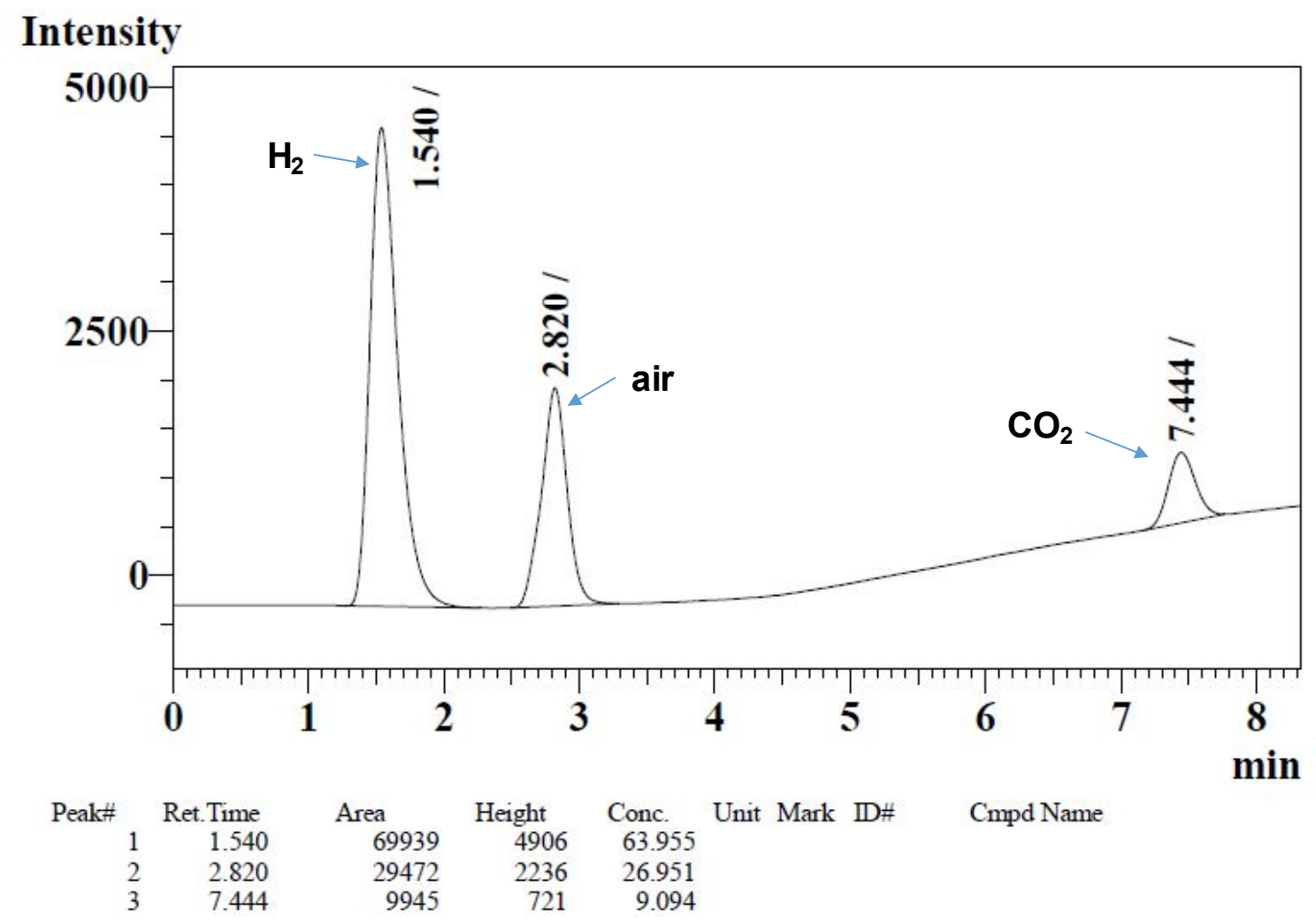

Figure S14.GC-TCD analysis of $(a)$ evolved gas $\left(\mathrm{H}_{2}: \mathrm{CO}_{2} \approx 1: 1\right)$ gas after complete dehydrogenation of formic acid with [Ru]-1. Reaction condition: [Ru]-1(0.01 mmol), formic acid $(0.4 \mathrm{M}, 2.5 \mathrm{~mL})$, sodium formate $(0.05 \mathrm{mmol}), 90{ }^{\circ} \mathrm{C}$. (Analysis is performed using Argon as the carrier gas). 
(a)

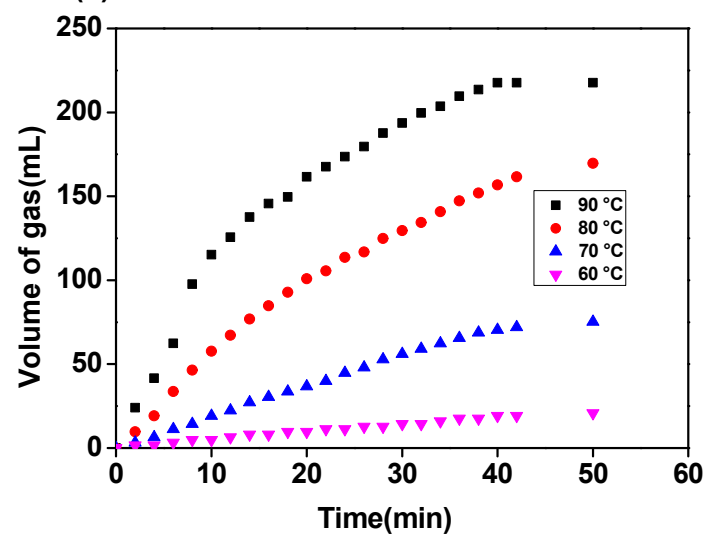

(b)

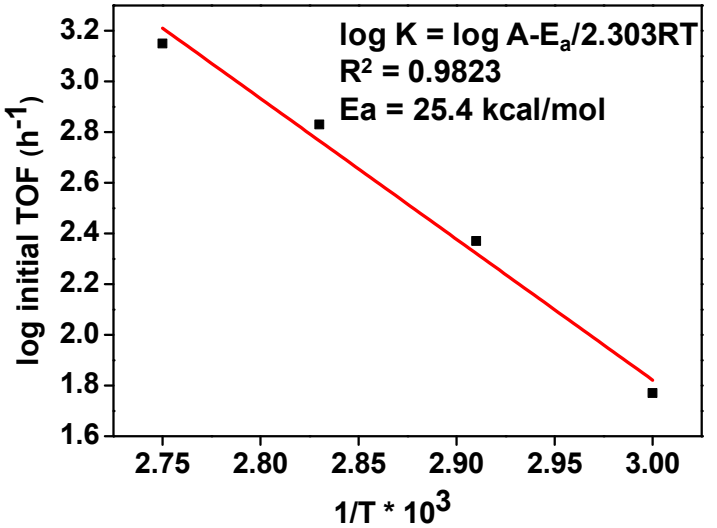

Figure S15.(a) Temperature dependent formic acid dehydrogenation over [Ru]-1, and the corresponding (b) Arrhenius plot of the initial TOF values for formic acid (2M, $2.5 \mathrm{~mL})$ dehydrogenation over $[\mathrm{Ru}]-1(0.2 \mathrm{~mol} \%),[\mathrm{HCOONa}] /[\mathrm{HCOOH}]=2: 1,60^{\circ} \mathrm{C}$ $-90^{\circ} \mathrm{C}$.

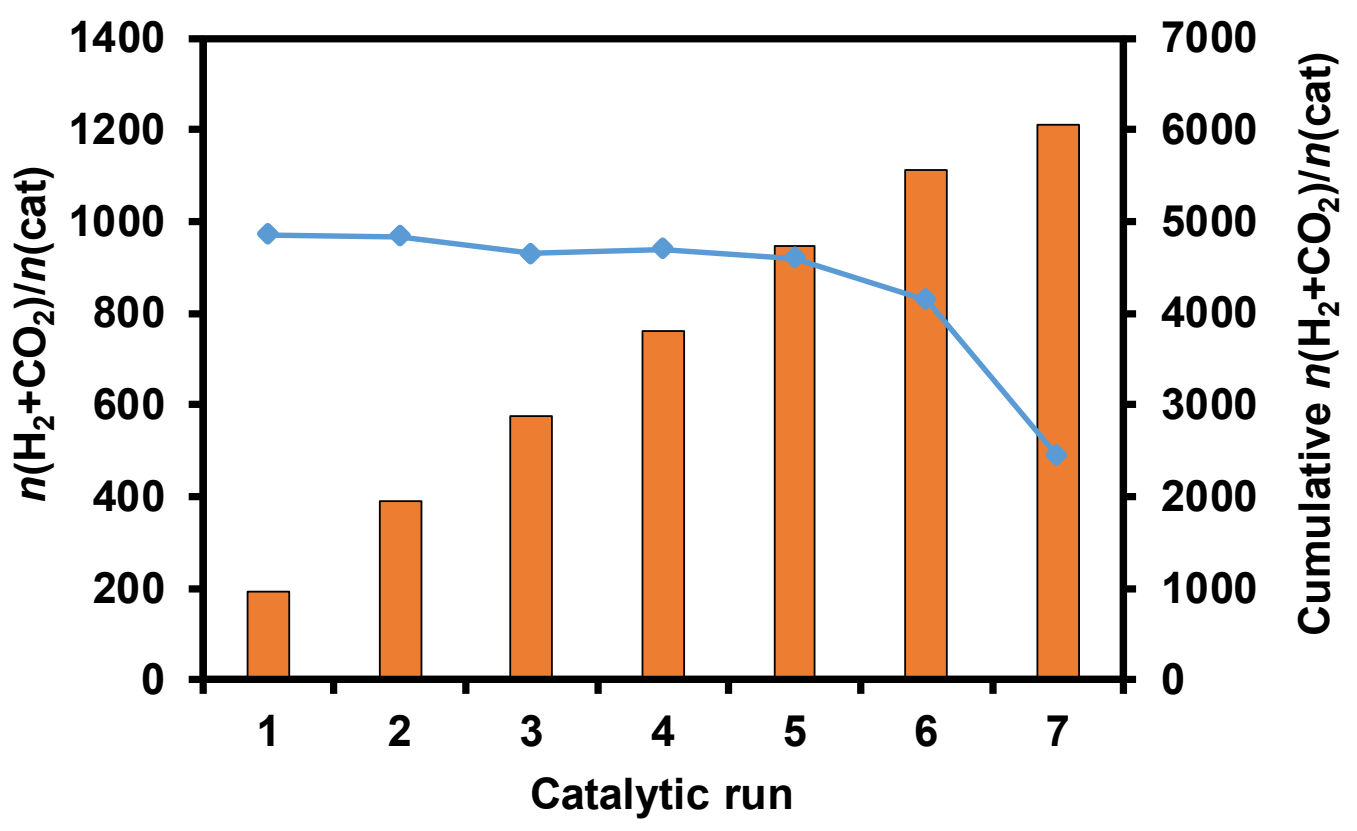

Figure S16. Recycling experiment for the catalytic dehydrogenation of formic acid over [Ru]-1. Reaction condition: formic acid $(4.0 \mathrm{M}, 2.5 \mathrm{~mL})$, [Ru]-1 (0.1 mol\%), $[\mathrm{HCOONa}] /[\mathrm{HCOOH}]=2: 1,90^{\circ} \mathrm{C}$. $(10 \mathrm{mmol}$ of formic acid is added to the reaction mixture after each run). 


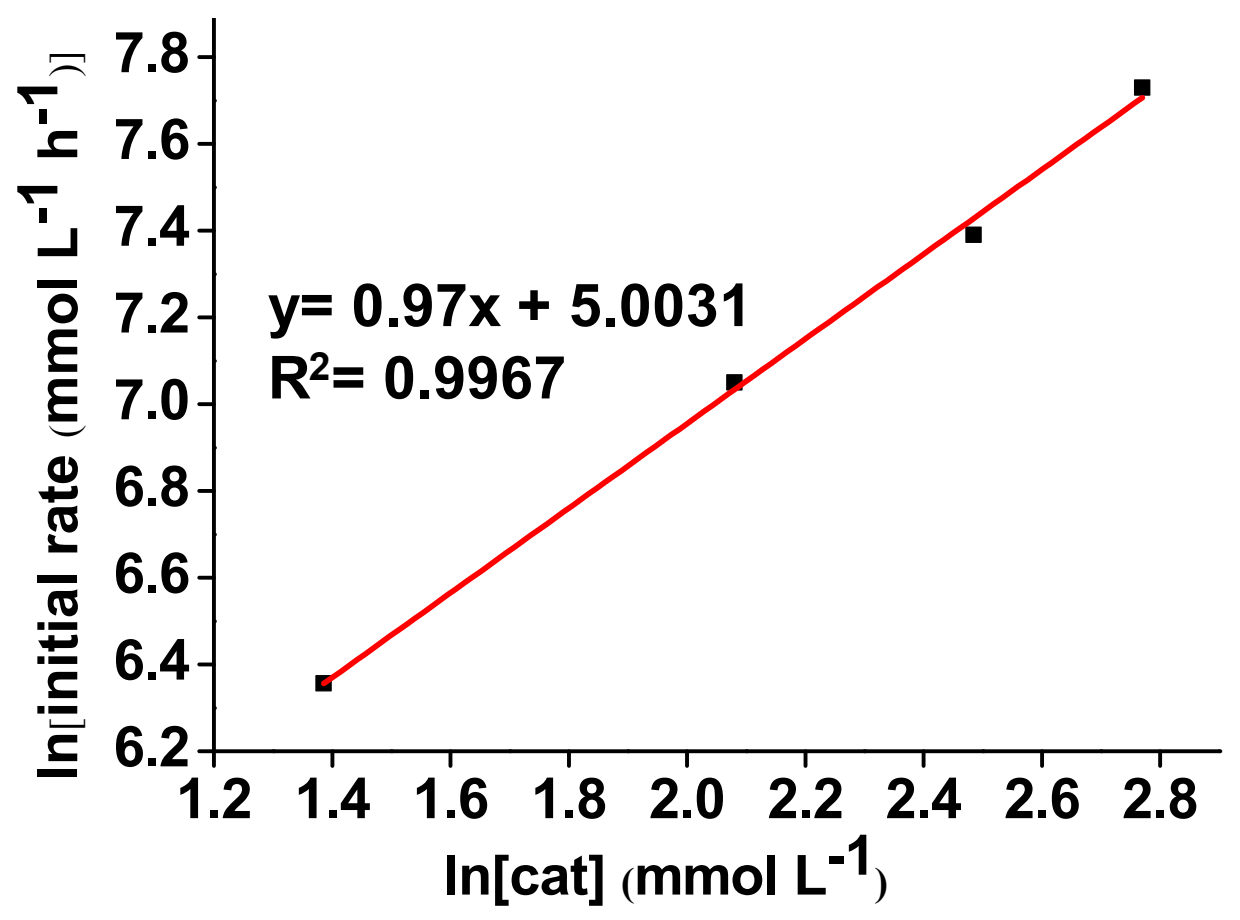

Figure S17. Plot of $\operatorname{In}\left[\right.$ initial rate $\left.\left(\mathrm{mmolL}^{-1} \mathrm{~h}^{-1}\right)\right]$ vs $\ln [$ cat $]\left(\mathrm{mmol} \mathrm{L}^{-1}\right)$. Reaction condition: [Ru]-1 (0.01 mmol-0.04 mmol), formic acid (1.0 M, $2.5 \mathrm{~mL}), 90^{\circ} \mathrm{C}$.

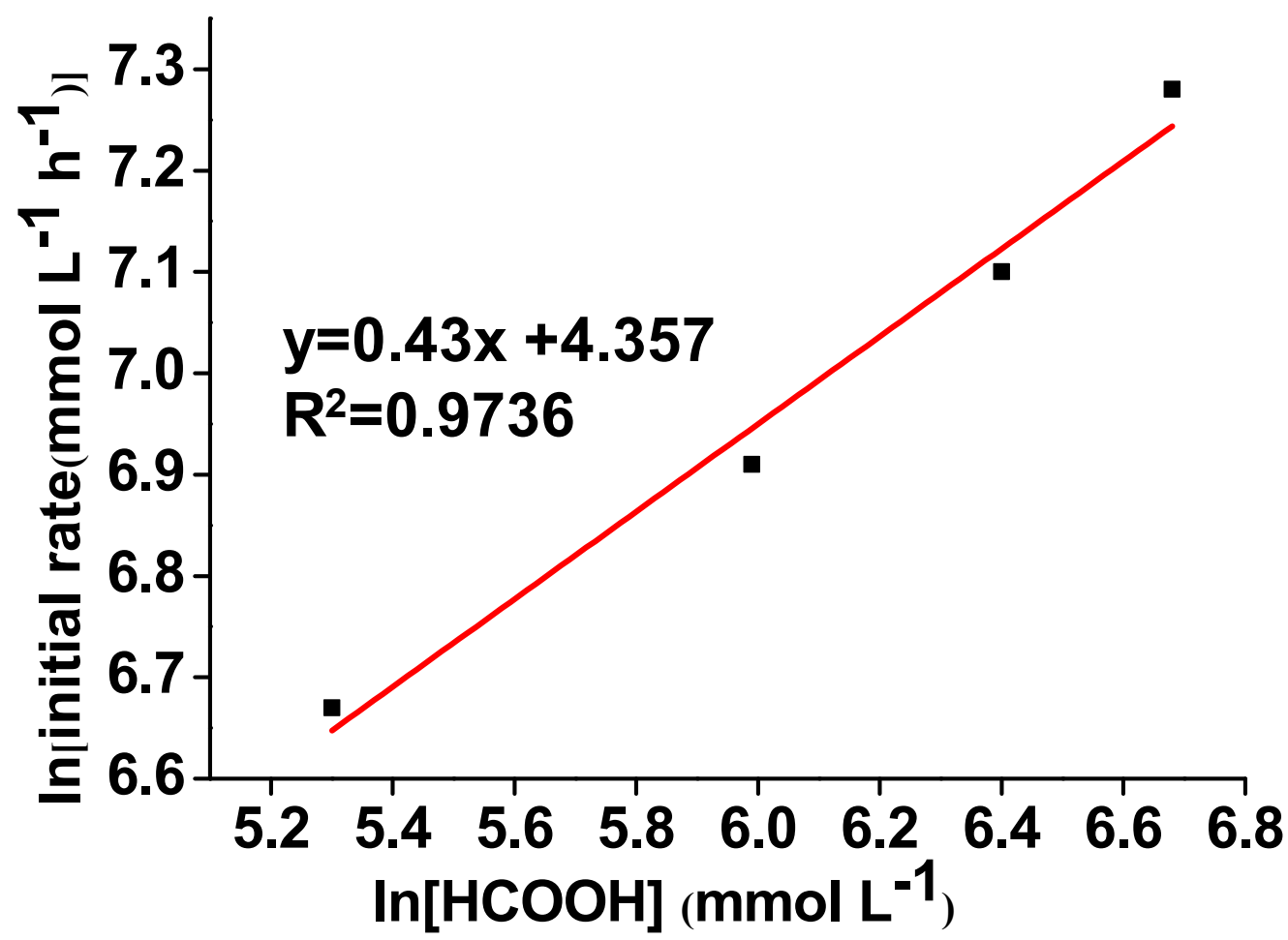

Figure S18. Plot of In[initial rate $\left.\left(\mathrm{mmolL}^{-1} \mathrm{~h}^{-1}\right)\right]$ vs $\operatorname{In}[\mathrm{HCOOH}]\left(\mathrm{mmol} \mathrm{L} \mathrm{L}^{-1}\right)$. Reaction condition: [Ru]-1 (0.01 mmol), formic acid $(0.2-0.8 \mathrm{M}, 2.5 \mathrm{~mL}), 90^{\circ} \mathrm{C}$. 


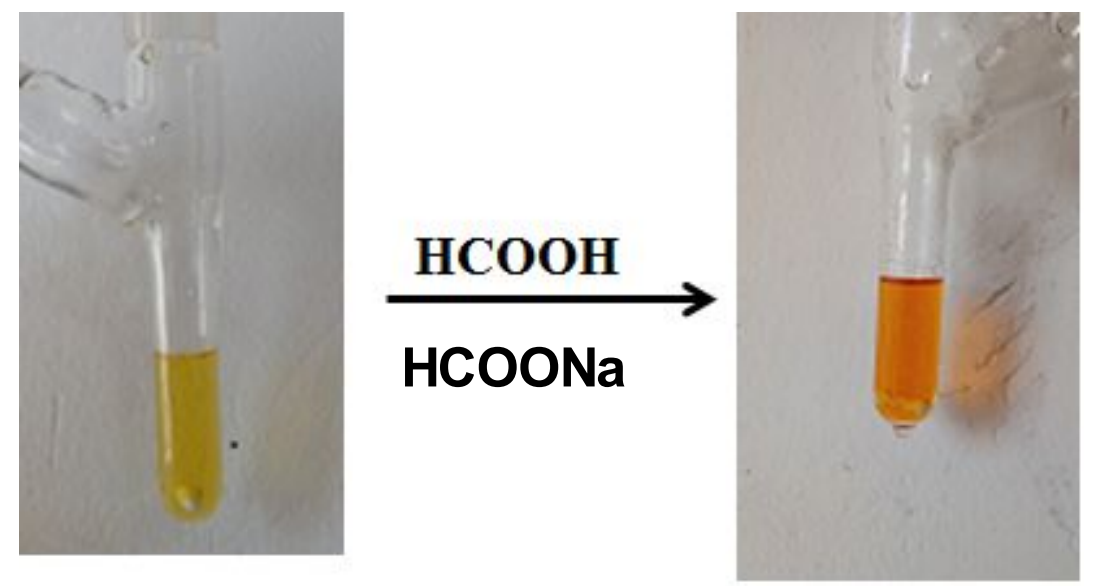

Figure S19. Transformation of colour of the reaction mixture during the catalytic dehydrogenation of formic acid. Reaction Condition: [Ru]-1 $(0.01 \mathrm{mmol})$, formic acid $(0.4 \mathrm{M}, 2.5 \mathrm{~mL})$, sodium formate $(0.05 \mathrm{mmol}), \mathrm{T}=90^{\circ} \mathrm{C}$.

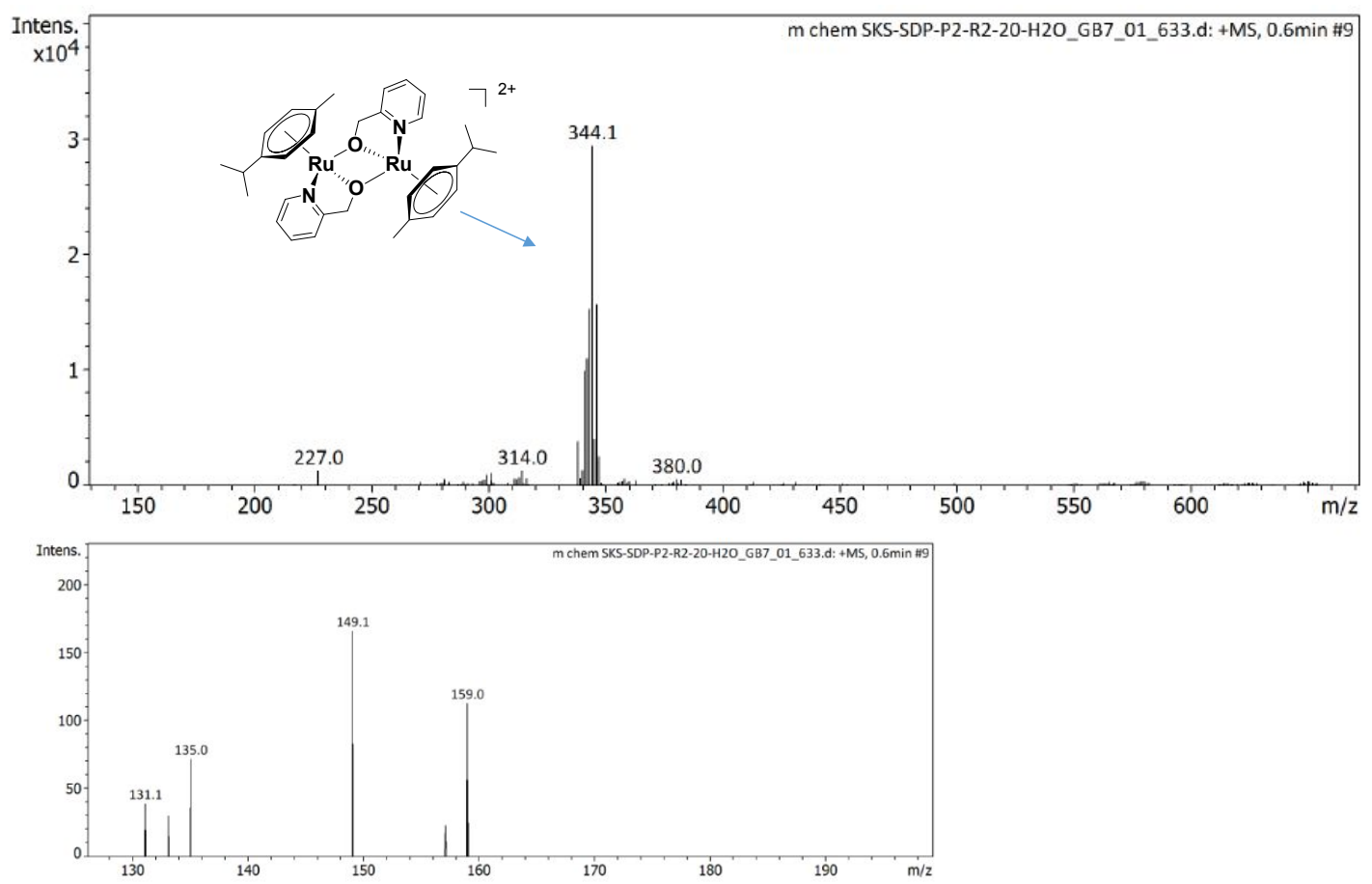

Figure S20. Mass spectra of the reaction aliquot for the base assisted catalytic dehydrogenation of formic acid after 5 minutes. Reaction condition: [Ru]-1 (0.01 $\mathrm{mmol})$, formic acid $(0.4 \mathrm{M}, 2.5 \mathrm{~mL})$, sodium formate $(0.05 \mathrm{mmol}), \mathrm{T}=90^{\circ} \mathrm{C}$. 


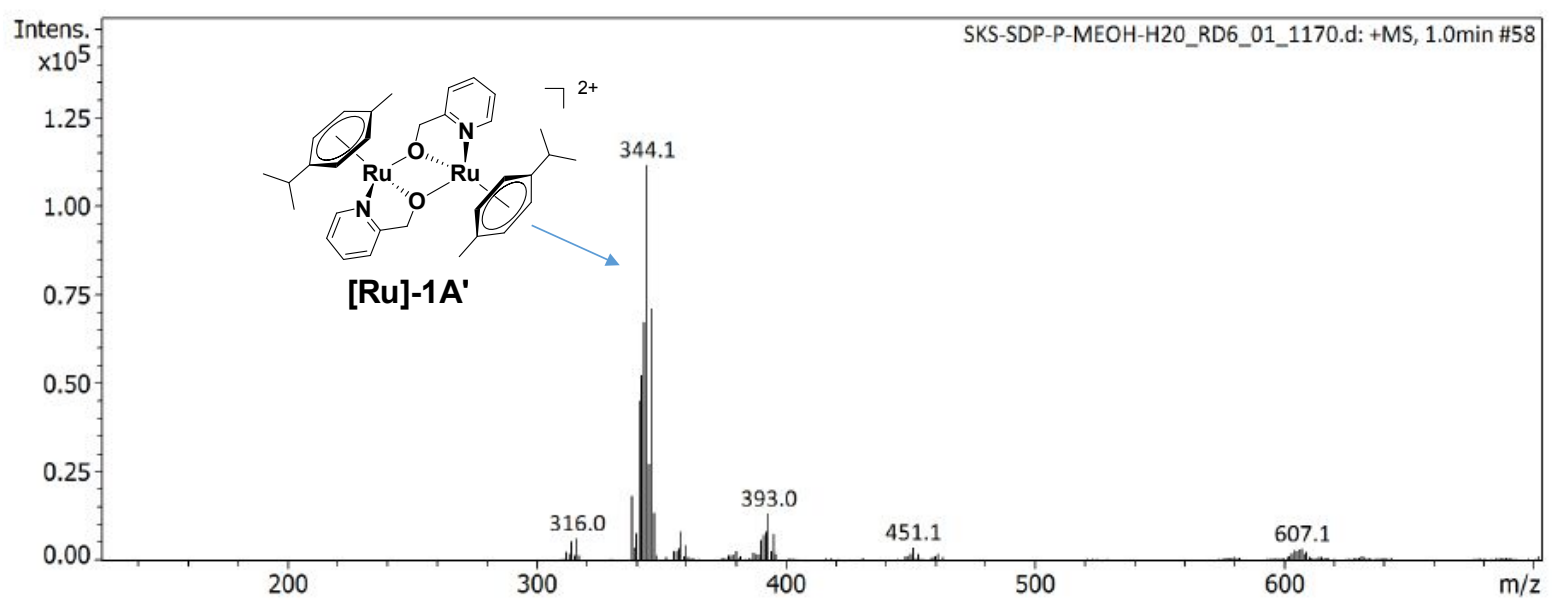

Figure S21. Mass spectra of the reaction aliquot at the end of the base assisted catalytic dehydrogenation of formic acid. Reaction condition: [Ru]-1 $(0.01 \mathrm{mmol})$, formic acid $(0.4 \mathrm{M}, 2.5 \mathrm{~mL})$, sodium formate $(0.05 \mathrm{mmol}), \mathrm{T}=90^{\circ} \mathrm{C}$.

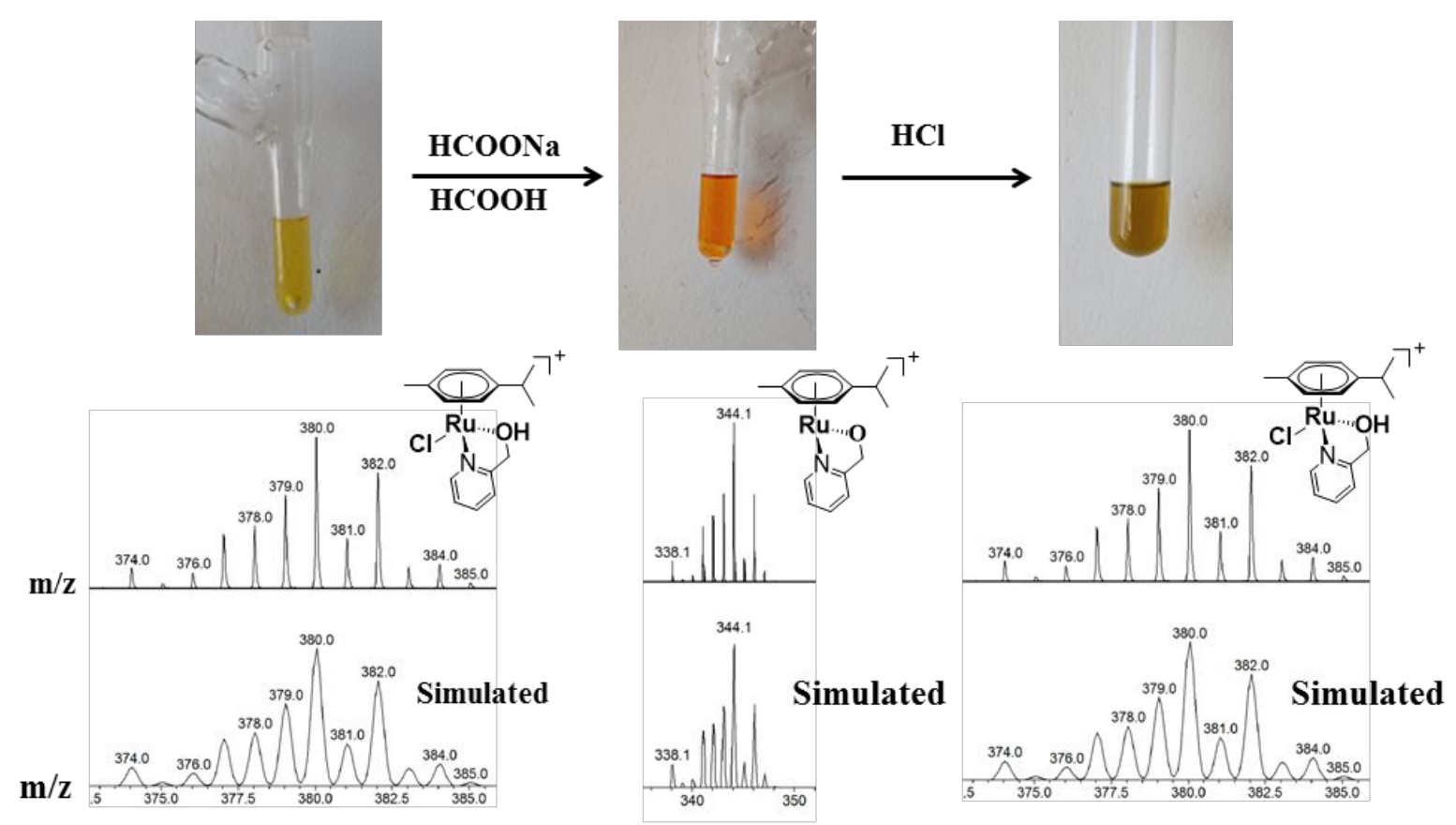

Figure S22. Mass investigation of the reaction mixture for catalytic dehydrogenation of formic acid over [Ru]-1 catalyst showing the colour change from the initial yellow to red-orange during the initial minutes and reverts back to yellow colour when treated with an excess of dil. $\mathrm{HCl}$. 


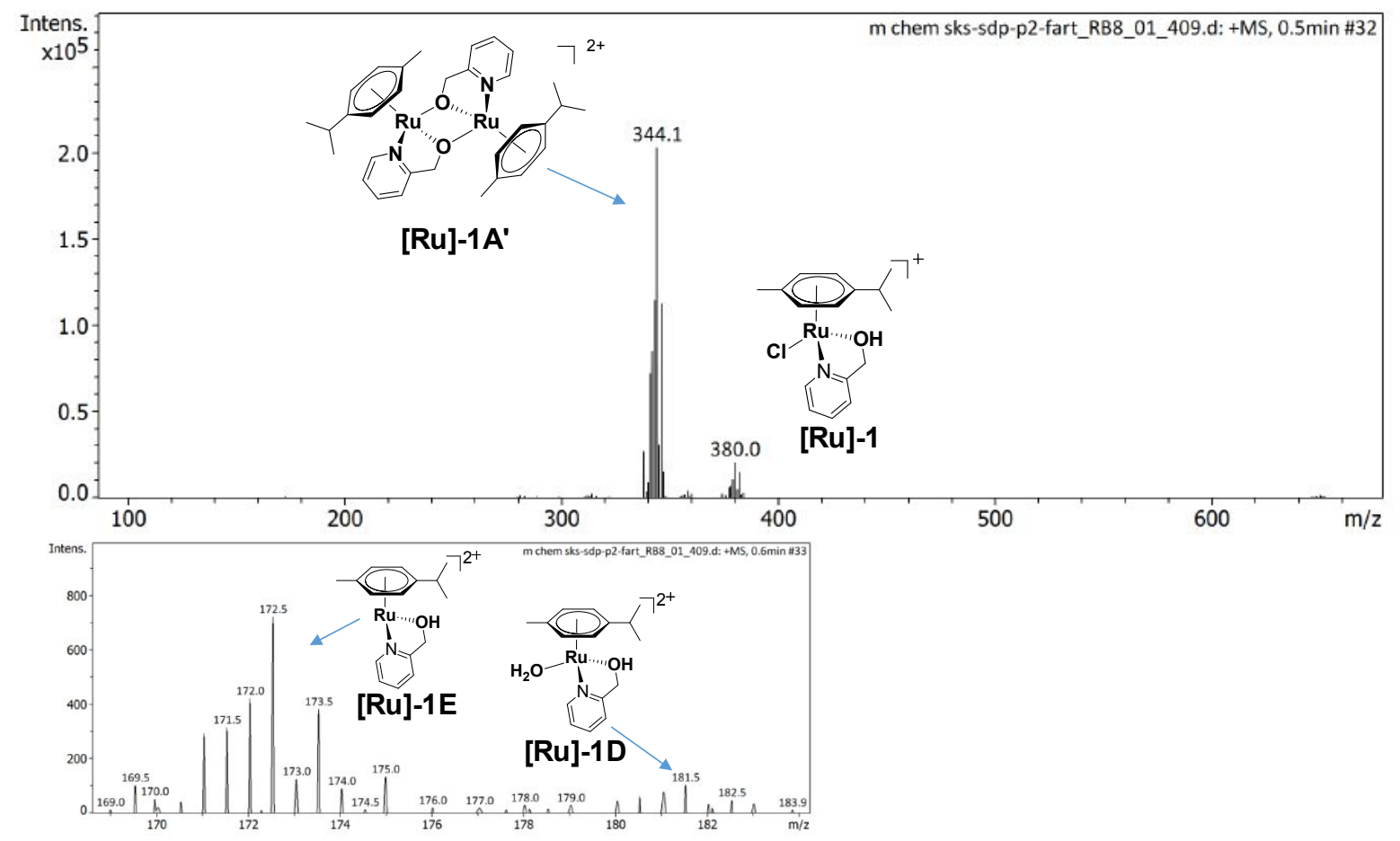

Figure S23. Mass spectra of the reaction aliquot for the reaction of [Ru]-1 $(0.01$ $\mathrm{mmol})$ with formic acid $(0.4 \mathrm{M}, 2.5 \mathrm{~mL})$ added to it and stirred at room temperature for 10 minutes.
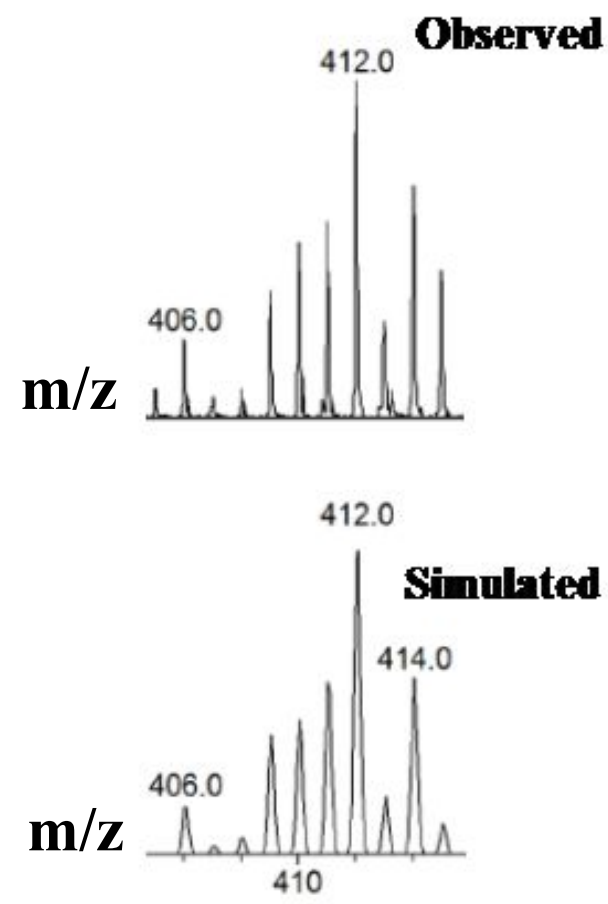

Figure S24. Observation of ruthenium-formate species [Ru]-1B (m/z $\left.412[\mathrm{M}]+\mathrm{Na}^{+}\right)$ observed during mass investigation. Reaction condition: [Ru]-1 $(0.01 \mathrm{mmol})$, formic acid $(0.2 \mathrm{M}, 2.5 \mathrm{~mL})$, sodium formate $(0.05 \mathrm{mmol})$ and stirred at room temperature for 10 minutes. 


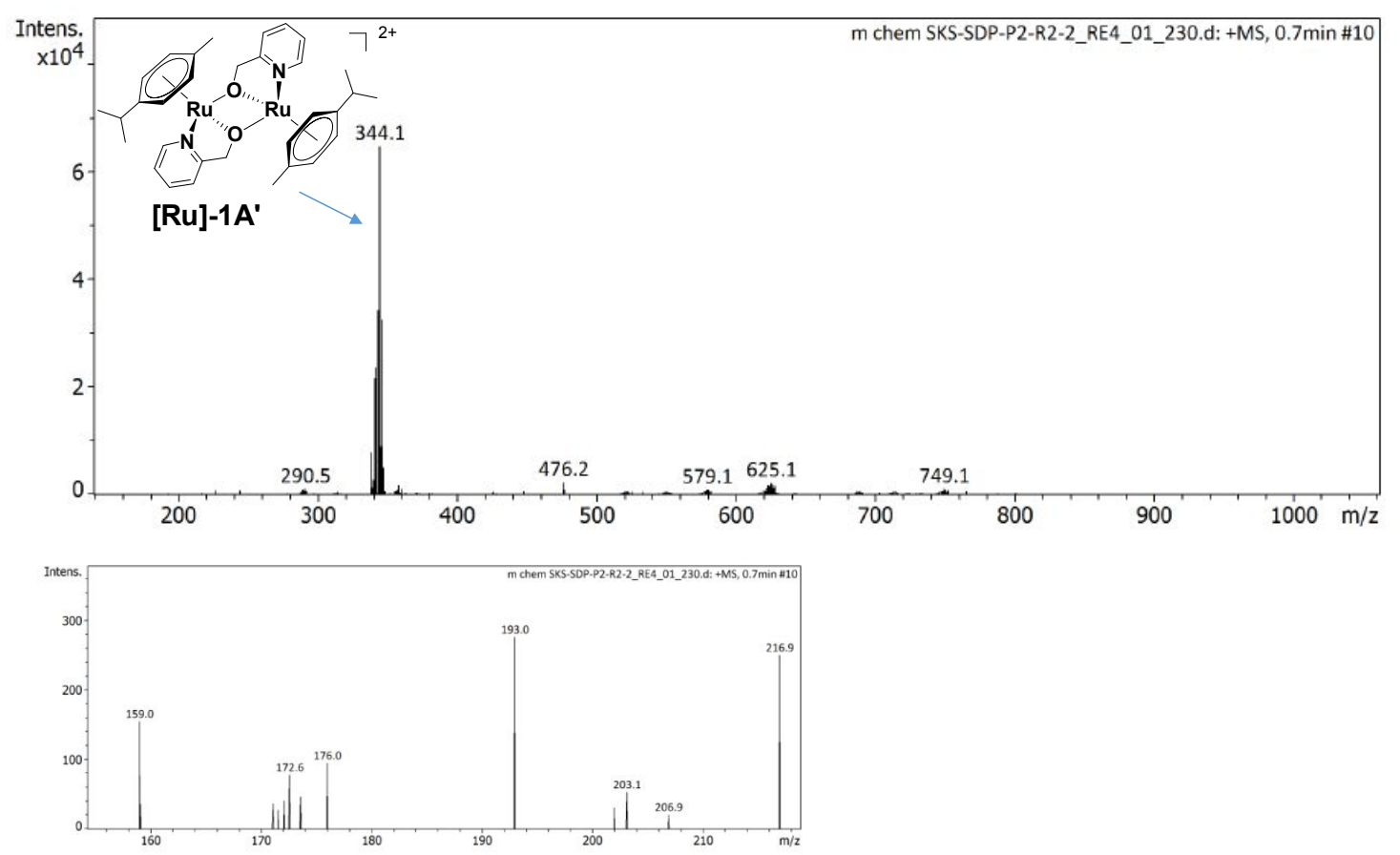

Figure S25. Mass spectra of the reaction aliquot for the reaction of [Ru]-1 $(0.01$ $\mathrm{mmol}$ ) with sodium formate $(0.04 \mathrm{M}, 2.5 \mathrm{~mL})$ at room temperature for 10 minutes.

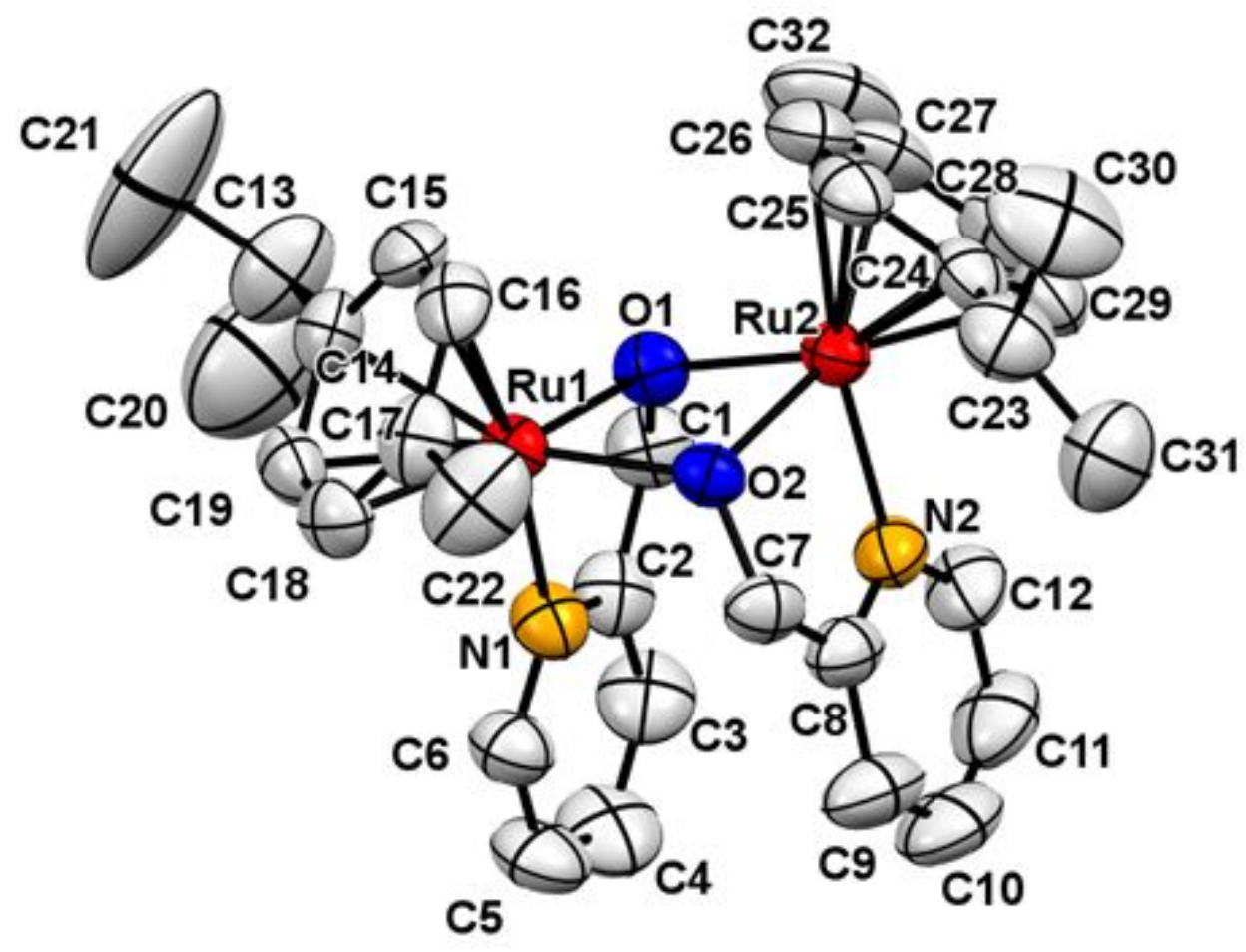

Figure S26. Single crystal X-ray molecular structure of [Ru]-1A'. Counter anions $\left(\mathrm{Cl}^{-}\right.$ ) and all hydrogen atoms of [Ru]-1A' are omitted for clarity. Selected bond lengths $(\AA)$ and angles $\left({ }^{\circ}\right): \mathrm{Ru}(2)-\mathrm{N}(2) 2.080(5), \mathrm{Ru}(2)-\mathrm{O}(2)$ 2.082(4), $\mathrm{Ru}(2)-\mathrm{O}(1) 2.120(4)$, $\mathrm{Ru}(1)-\mathrm{O}(1)$ 2.093(4), N(2)-Ru(2)-O(2) 77.83(19), N(2)-Ru(2)-O(1) 90.20(2), O(2)$\mathrm{Ru}(2)-\mathrm{O}(1)$ 76.91(15), N(1)-Ru(1)-O(1) 78.5(2). 


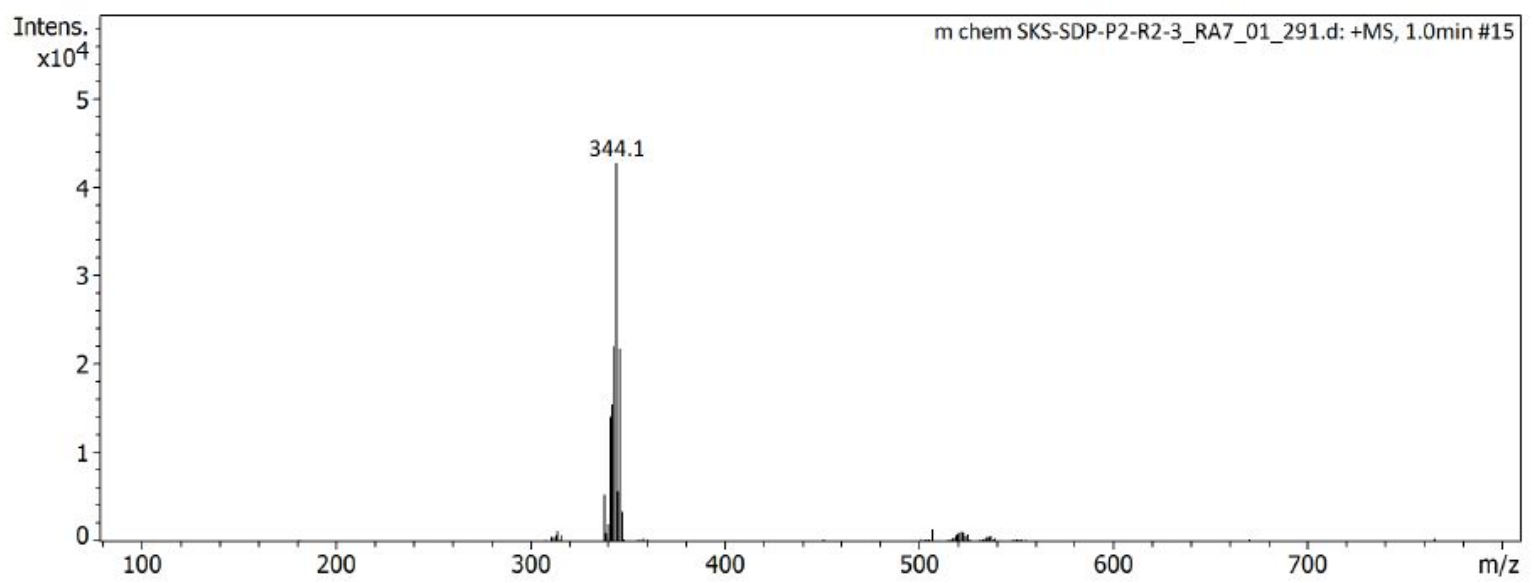

Figure S27. Mass spectra of [Ru]-1A'

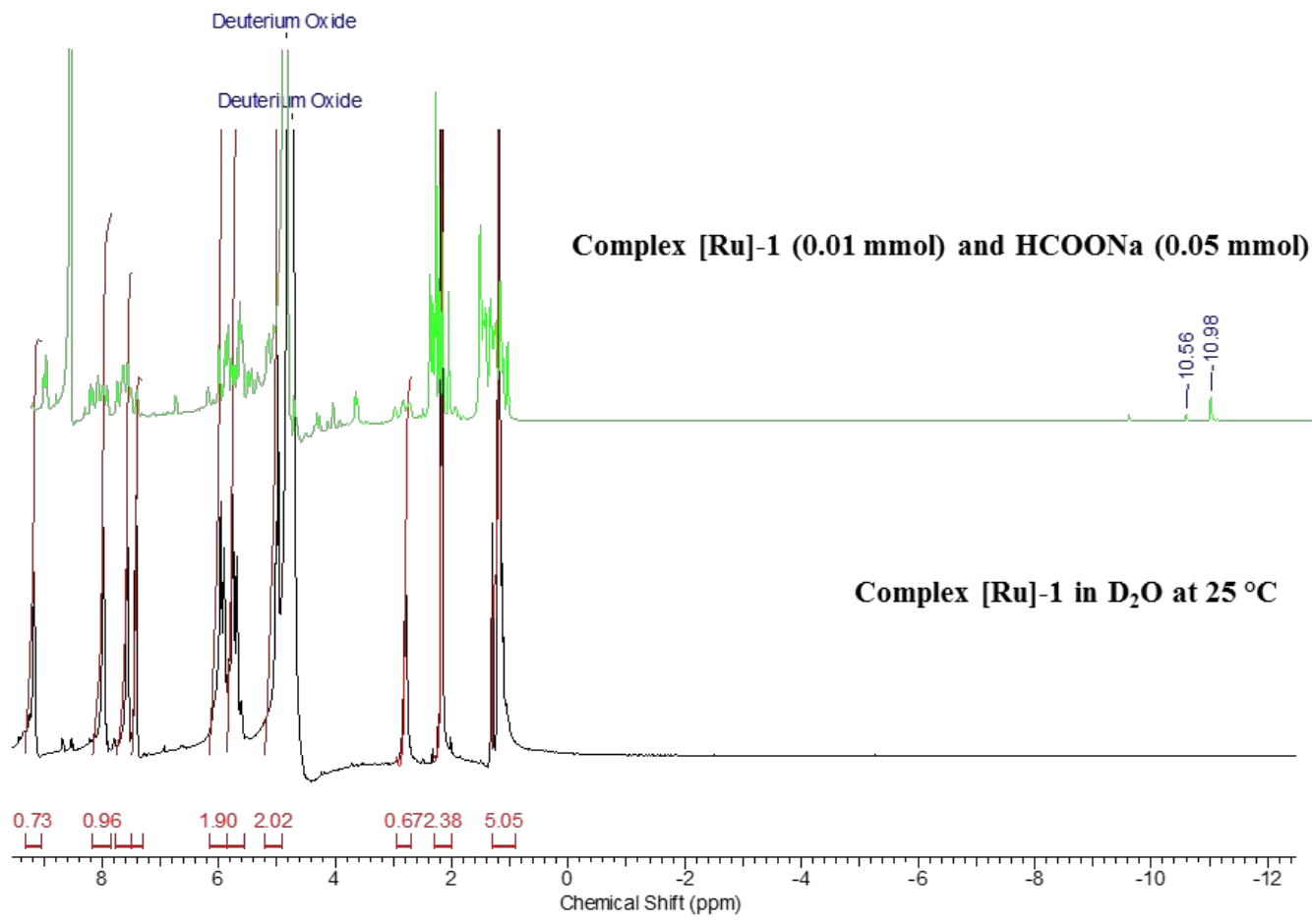

Figure S28. ${ }^{1} \mathrm{H}$ NMR spectra showing the generation of Ru-hydride species during the treatment of $[R u]-1(0.01 \mathrm{mmol})$ with sodium formate $(0.05 \mathrm{mmol})$ in $\mathrm{D}_{2} \mathrm{O}(0.6$ $\mathrm{mL}$ ) at $25^{\circ} \mathrm{C}$ 
Table S6. Single crystal X-ray refinement data for [Ru]-1A'

\begin{tabular}{|c|c|}
\hline Formula & $\mathrm{C}_{32} \mathrm{H}_{40} \mathrm{~F}_{12} \mathrm{~N}_{2} \mathrm{O}_{2} \mathrm{P}_{2} \mathrm{Ru}_{2}$ \\
\hline Molecular weight & 976.74 \\
\hline Crystal system & Triclinic \\
\hline Space group & P-1 \\
\hline Temperature/K & 293 \\
\hline Wavelength & 0.71073 \\
\hline$a / \AA$ & $11.3876(13)$ \\
\hline$b / \AA$ & $12.6247(18)$ \\
\hline$c / \AA$ & $14.534(2)$ \\
\hline$\alpha /^{\circ}$ & $82.822(6)$ \\
\hline$\beta /^{\circ}$ & $83.484(5)$ \\
\hline$\gamma /{ }^{\circ}$ & $75.943(5)$ \\
\hline $\mathrm{V} / \AA^{3}$ & $2003.5(5)$ \\
\hline Z & 2 \\
\hline $\begin{array}{l}\text { Density } / \mathrm{gcm}^{-1} \\
\text { Absorption Coefficient }\end{array}$ & $\begin{array}{l}1.619 \\
0.920\end{array}$ \\
\hline $\mathrm{F}(000)$ & 976 \\
\hline Total no of reflections & 7234 \\
\hline Reflections, $I>2 \sigma(I)$ & 6189 \\
\hline $\operatorname{Max} .2 \theta /{ }^{\circ}$ & 25.25 \\
\hline Ranges (h, k, l) & $\begin{array}{l}-13 \leq \mathrm{h} \leq 13 \\
-15 \leq \mathrm{k} \leq 15 \\
-17 \leq 1 \leq 17\end{array}$ \\
\hline Complete to $2 \theta(\%)$ & $99.5 \%$ \\
\hline Refinement method & $\begin{array}{l}\text { Full-matrix least- } \\
\text { squares on } F^{2}\end{array}$ \\
\hline $\operatorname{Goof}\left(F^{2}\right)$ & 1.071 \\
\hline $\mathrm{R}$ indices $[I>2 \sigma(I)]$ & 0.0541 \\
\hline $\mathrm{R}$ Indices (all data) & 0.0636 \\
\hline
\end{tabular}

Table S7. Selected bond lengths $(\AA)$ for complex [Ru]-1A'

\begin{tabular}{ll}
\hline $\mathrm{Ru}(2)-\mathrm{N}(2)$ & $2.080(5)$ \\
$\mathrm{Ru}(2)-\mathrm{O}(2)$ & $2.082(4)$ \\
\hline
\end{tabular}




\begin{tabular}{ll}
\hline $\mathrm{Ru}(2)-\mathrm{O}(1)$ & $2.120(4)$ \\
$\mathrm{Ru}(2)-\mathrm{C}(25)$ & $2.172(6)$ \\
$\mathrm{Ru}(2)-\mathrm{C}(28)$ & $2.178(7)$ \\
$\mathrm{Ru}(2)-\mathrm{C}(24)$ & $2.181(6)$ \\
$\mathrm{Ru}(2)-\mathrm{C}(26)$ & $2.187(7)$ \\
$\mathrm{Ru}(2)-\mathrm{C}(29)$ & $2.187(7)$ \\
$\mathrm{Ru}(2)-\mathrm{C}(27)$ & $2.192(7)$ \\
$\mathrm{Ru}(1)-\mathrm{N}(1)$ & $2.084(5)$ \\
$\mathrm{Ru}(1)-\mathrm{O}(1)$ & $2.093(4)$ \\
$\mathrm{Ru}(1)-\mathrm{O}(2)$ & $2.103(4)$ \\
$\mathrm{Ru}(1)-\mathrm{C}(15)$ & $2.169(6)$ \\
$\mathrm{Ru}(1)-\mathrm{C}(19)$ & $2.171(6)$ \\
$\mathrm{Ru}(1)-\mathrm{C}(18)$ & $2.178(6)$ \\
$\mathrm{Ru}(1)-\mathrm{C}(16)$ & $2.188(6)$ \\
$\mathrm{Ru}(1)-\mathrm{C}(14)$ & $2.191(7)$ \\
$\mathrm{Ru}(1)-\mathrm{C}(17)$ & $2.205(6)$ \\
$\mathrm{O}(2)-\mathrm{C}(7)$ & $1.419(7)$ \\
$\mathrm{O}(1)-\mathrm{C}(1)$ & $1.417(8)$ \\
$\mathrm{N}(1)-\mathrm{C}(6)$ & $1.323(9)$ \\
$\mathrm{N}(1)-\mathrm{C}(2)$ & $1.362(9)$ \\
$\mathrm{N}(2)-\mathrm{C}(12)$ & $1.325(9)$ \\
$\mathrm{N}(2)-\mathrm{C}(8)$ & $1.340(9)$ \\
\hline
\end{tabular}

Table S8. Selected bond angles $\left({ }^{\circ}\right)$ for complex [Ru]-1A'

\begin{tabular}{cc}
\hline $\mathrm{N}(2)-\mathrm{Ru}(2)-\mathrm{O}(2)$ & $77.83(19)$ \\
$\mathrm{N}(2)-\mathrm{Ru}(2)-\mathrm{O}(1)$ & $90.20(2)$ \\
$\mathrm{O}(2)-\mathrm{Ru}(2)-\mathrm{O}(1)$ & $76.91(15)$ \\
$\mathrm{N}(2)-\mathrm{Ru}(2)-\mathrm{C}(25)$ & $147.9(3)$ \\
$\mathrm{O}(2)-\mathrm{Ru}(2)-\mathrm{C}(25)$ & $93.8(2)$ \\
$\mathrm{O}(1)-\mathrm{Ru}(2)-\mathrm{C}(25)$ & $118.4(2)$ \\
$\mathrm{N}(2)-\mathrm{Ru}(2)-\mathrm{C}(28)$ & $100.2(3)$ \\
$\mathrm{O}(2)-\mathrm{Ru}(2)-\mathrm{C}(25)$ & $165.1(3)$ \\
$\mathrm{O}(1)-\mathrm{Ru}(2)-\mathrm{C}(28)$ & $118.0(3)$
\end{tabular}




\begin{tabular}{|c|c|}
\hline $\mathrm{N}(2)-\mathrm{Ru}(2)-\mathrm{C}(24)$ & $111.6(3)$ \\
\hline $\mathrm{O}(2)-\mathrm{Ru}(2)-\mathrm{C}(24)$ & $98.1(2)$ \\
\hline $\mathrm{O}(1)-\mathrm{Ru}(2)-\mathrm{C}(24)$ & $156.4(2)$ \\
\hline $\mathrm{N}(2)-\mathrm{Ru}(2)-\mathrm{C}(26)$ & $166.9(3)$ \\
\hline $\mathrm{O}(2)-\mathrm{Ru}(2)-\mathrm{C}(26)$ & $115.3(3)$ \\
\hline $\mathrm{O}(1)-\mathrm{Ru}(2)-\mathrm{C}(26)$ & $92.1(3)$ \\
\hline $\mathrm{N}(2)-\mathrm{Ru}(2)-\mathrm{C}(29)$ & $92.1(3)$ \\
\hline $\mathrm{O}(2)-\mathrm{Ru}(2)-\mathrm{C}(29)$ & $127.0(2)$ \\
\hline $\mathrm{O}(1)-\mathrm{Ru}(2)-\mathrm{C}(29)$ & $155.9(2)$ \\
\hline $\mathrm{N}(2)-\mathrm{Ru}(2)-\mathrm{C}(27)$ & $129.5(3)$ \\
\hline $\mathrm{O}(2)-\mathrm{Ru}(2)-\mathrm{C}(27)$ & $150.7(3)$ \\
\hline $\mathrm{O}(1)-\mathrm{Ru}(2)-\mathrm{C}(27)$ & $90.9(3)$ \\
\hline $\mathrm{N}(1)-\mathrm{Ru}(1)-\mathrm{O}(1)$ & $78.5(2)$ \\
\hline $\mathrm{N}(1)-\mathrm{Ru}(1)-\mathrm{O}(2)$ & $89.8(19)$ \\
\hline $\mathrm{O}(1)-\mathrm{Ru}(1)-\mathrm{O}(2)$ & $77.03(16)$ \\
\hline $\mathrm{N}(1)-\mathrm{Ru}(1)-\mathrm{C}(15)$ & $150.0(3)$ \\
\hline $\mathrm{O}(1)-\mathrm{Ru}(1)-\mathrm{C}(15)$ & $95.4(2)$ \\
\hline $\mathrm{O}(2)-\mathrm{Ru}(1)-\mathrm{C}(15)$ & $117.7(2)$ \\
\hline $\mathrm{N}(1)-\mathrm{Ru}(1)-\mathrm{C}(19)$ & $93.7(2)$ \\
\hline $\mathrm{O}(1)-\mathrm{Ru}(1)-\mathrm{C}(19)$ & $128.5(2)$ \\
\hline $\mathrm{O}(2)-\mathrm{Ru}(1)-\mathrm{C}(19)$ & $154.4(2)$ \\
\hline $\mathrm{N}(1)-\mathrm{Ru}(1)-\mathrm{C}(18)$ & $99.6(3)$ \\
\hline $\mathrm{O}(1)-\mathrm{Ru}(1)-\mathrm{C}(18)$ & $166.0(2)$ \\
\hline $\mathrm{O}(2)-\mathrm{Ru}(1)-\mathrm{C}(18)$ & $116.9(2)$ \\
\hline $\mathrm{N}(1)-\mathrm{Ru}(1)-\mathrm{C}(16)$ & $165.4(3)$ \\
\hline $\mathrm{O}(1)-\mathrm{Ru}(1)-\mathrm{C}(16)$ & $115.5(2)$ \\
\hline $\mathrm{O}(2)-\mathrm{Ru}(1)-\mathrm{C}(16)$ & $90.7(2)$ \\
\hline $\mathrm{N}(1)-\mathrm{Ru}(1)-\mathrm{C}(14)$ & $113.8(3)$ \\
\hline $\mathrm{O}(1)-\mathrm{Ru}(1)-\mathrm{C}(14)$ & $99.5(2)$ \\
\hline $\mathrm{O}(2)-\mathrm{Ru}(1)-\mathrm{C}(14)$ & $155.2(2)$ \\
\hline $\mathrm{N}(1)-\mathrm{Ru}(1)-\mathrm{C}(17)$ & $127.7(3)$ \\
\hline $\mathrm{O}(1)-\mathrm{Ru}(1)-\mathrm{C}(17)$ & $151.2(2)$ \\
\hline $\mathrm{O}(2)-\mathrm{Ru}(1)-\mathrm{C}(17)$ & $90.0(2)$ \\
\hline
\end{tabular}




\section{Time dependent GC-TCD analysis of aq. formaldehyde dehydrogenation:}

From the time dependent GC-TCD analysis of aq. formaldehyde dehydrogenation and using the standard calibration graph, it was observed that the ratio of $\mathrm{H}_{2}: \mathrm{CO}_{2} \approx$ 6:1 in the first 10 minutes of the catalytic reaction and then the $\mathrm{CO}_{2}$ content increased with time and the ratio of $\mathrm{H}_{2}: \mathrm{CO}_{2}$ was calculated to be 2.2:1 after 30 minutes and 2:1 at the end of the catalytic reaction. This finding is consistent with the the two step dehydrogenation pathway of aq. formaldehyde where at first, methanediol is dehydrogenated to formic acid with the release of one equivalent of $\mathrm{H}_{2}$ and subsequently formic acid is dehydrogenated to $\mathrm{H}_{2}$ and $\mathrm{CO}_{2}$ with the release of the second equivalent of $\mathrm{H}_{2}$ and one equivalent $\mathrm{CO}_{2}$ 
(a)
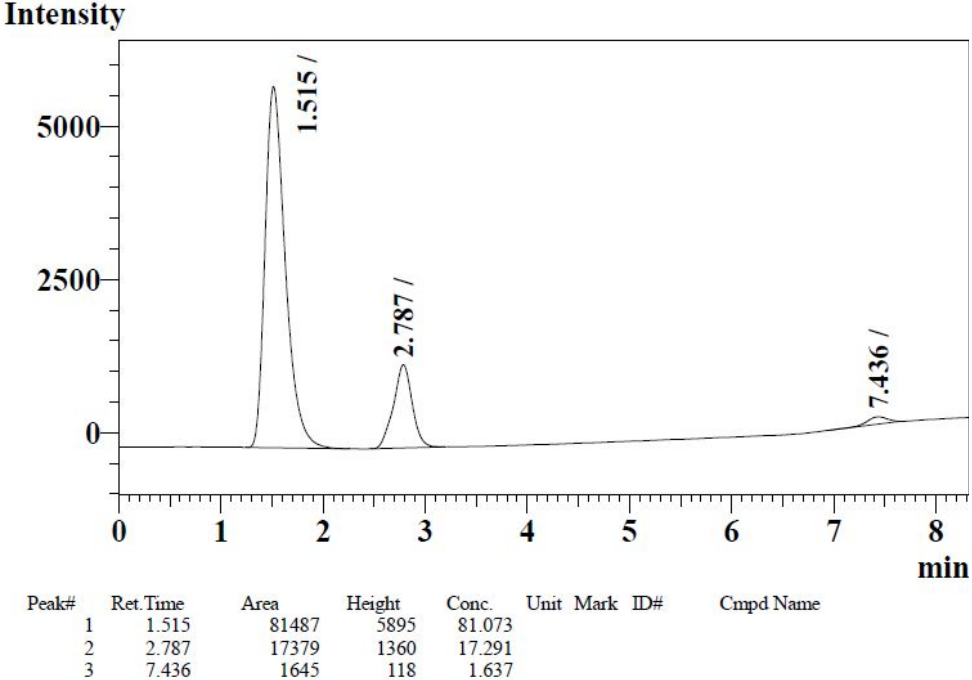

(b)

Intensity

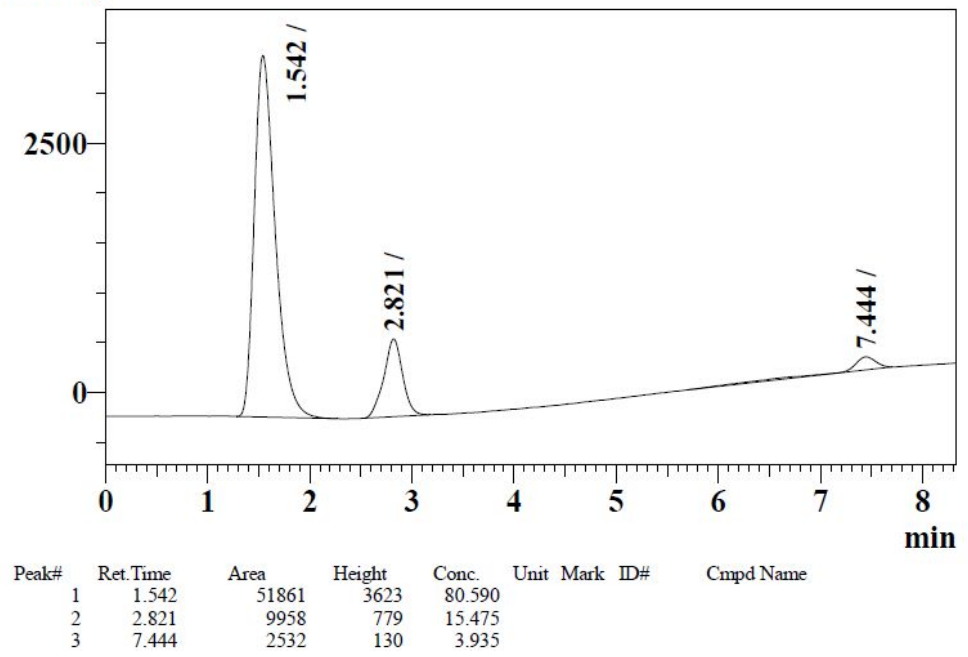

(c)

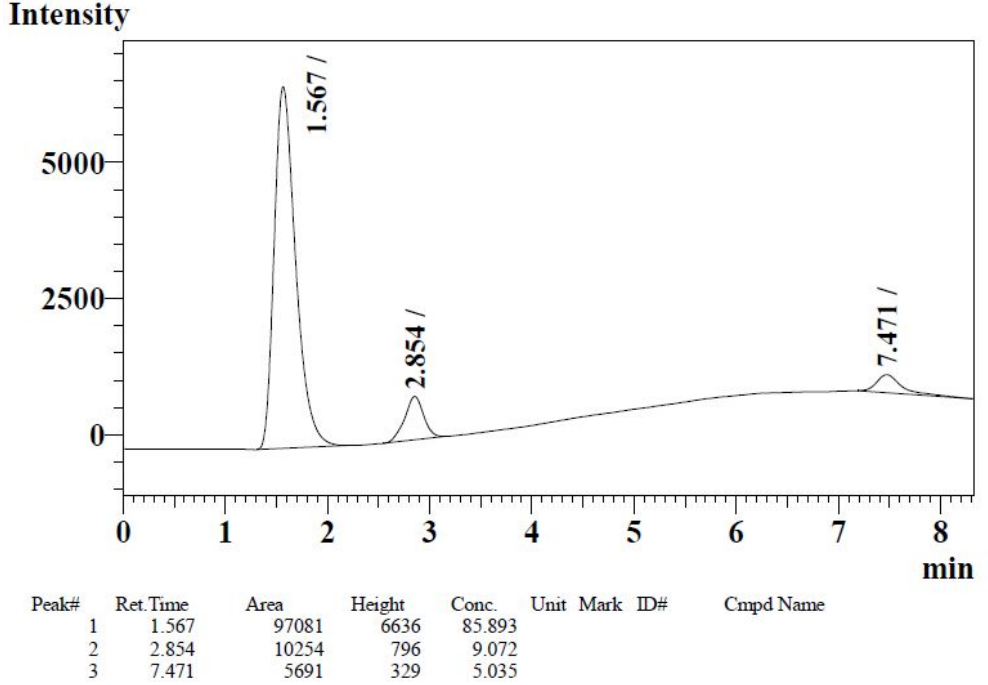

Figure S29.Time-dependent GC-TCD analysis of the evolved gas $\left(\mathrm{H}_{2}\right.$ and $\left.\mathrm{CO}_{2}\right)$ during the catalytic formaldehyde dehydrogenation reaction. (a) initial 10 minutes; $\mathrm{H}_{2}: \mathrm{CO}_{2} \approx 6: 1$ (b) After 30 minutes; $\mathrm{H}_{2}: \mathrm{CO}_{2} \approx 2.2: 1$. (c) After completion of reaction; $\mathrm{H}_{2}: \mathrm{CO}_{2} \approx 2: 1$. Reaction condition: [Ru]-1 (0.01 mmol), aq. formaldehyde $(0.4 \mathrm{M}$, $2.5 \mathrm{~mL}$ ), $90^{\circ} \mathrm{C}$. (Analysis is performed using Argon as the carrier gas). 
Time dependent $\mathrm{pH}$ analysis of aq. formaldehyde dehydrogenation:

From the time dependent $\mathrm{pH}$ analysis of the reaction medium during the catalytic dehydrogenation of aq. formaldehyde, it was found that the $\mathrm{pH}$ values slowly decreased from an initial $\mathrm{pH} \approx 4$ to 2.4 during the initial minutes and then slowly increased to almost the original $\mathrm{pH}$ at the commencement of the catalytic reaction. This observation is also consistent with the two step dehydrogenation pathway of aq. formaldehyde.

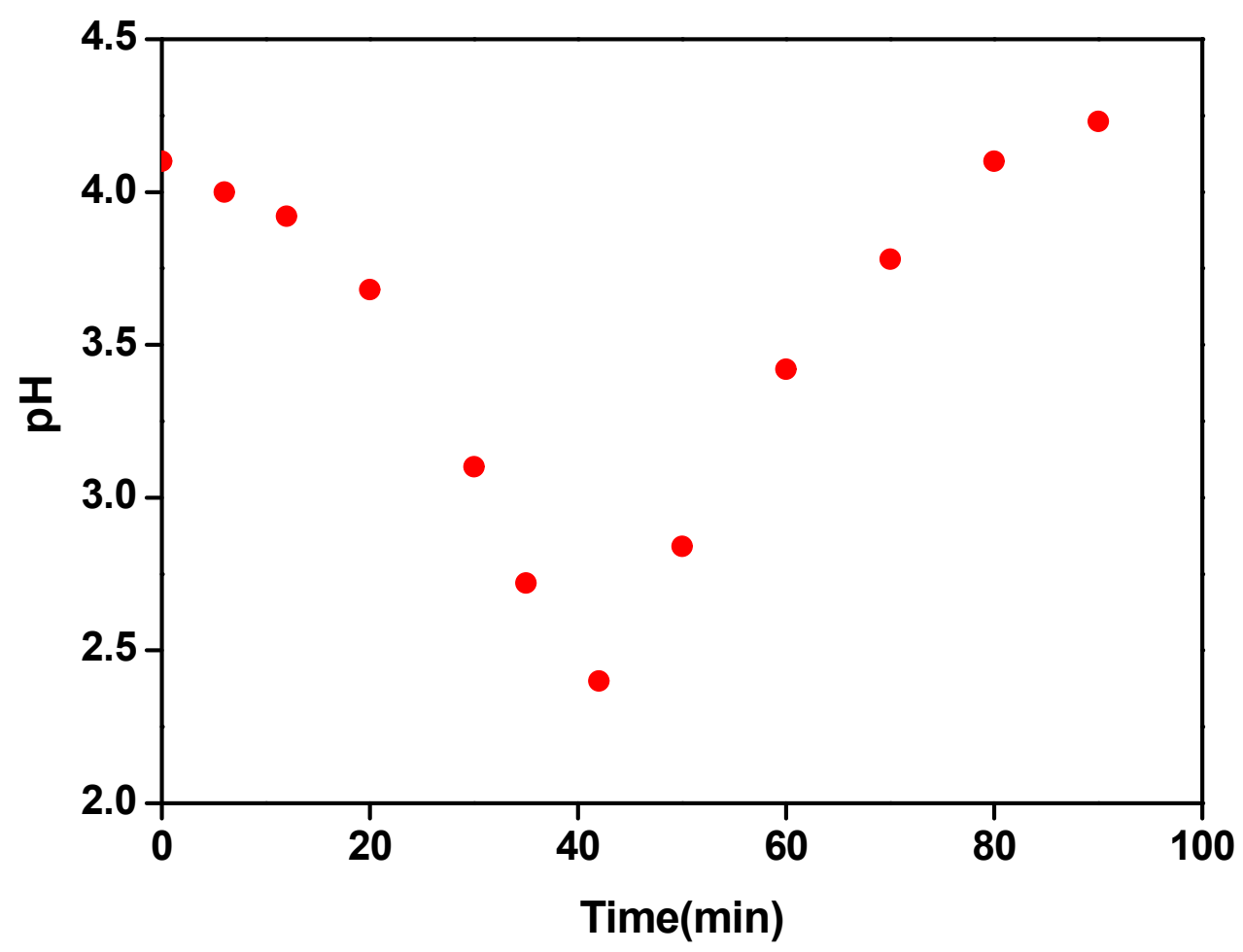

Figure S30.Time-dependent $\mathrm{pH}$ plot forthe catalytic formaldehyde dehydrogenation reaction. Reaction condition: $[R \mathbf{R u}-1(0.01 \mathrm{mmol})$, formaldehyde $(0.4 \mathrm{M}, 2.5 \mathrm{~mL}), 90$ ${ }^{\circ} \mathrm{C}$. 


$$
\mathrm{HCHO} \underset{\mathrm{H}_{2} \mathrm{O}, 90{ }^{\circ} \mathrm{C}}{\stackrel{[\mathrm{Ru}]-1}{\longrightarrow}} 2 \mathrm{H}_{2}+\mathrm{CO}_{2}
$$
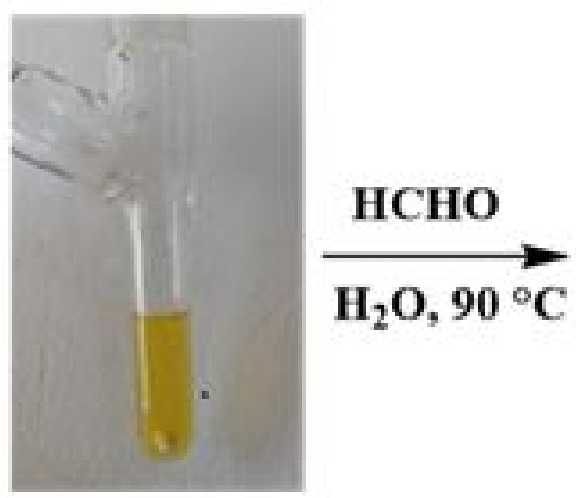

\section{$\mathbf{R u}-[1]$}

Figure S31. Transformation of colour of the reaction mixture during the catalytic dehydrogenation of aqueous formaldehyde. Reaction condition: [Ru]-1 (0.01 mmol), aq. formaldehyde $(0.4 \mathrm{M}, 2.5 \mathrm{~mL}), 90{ }^{\circ} \mathrm{C}$.

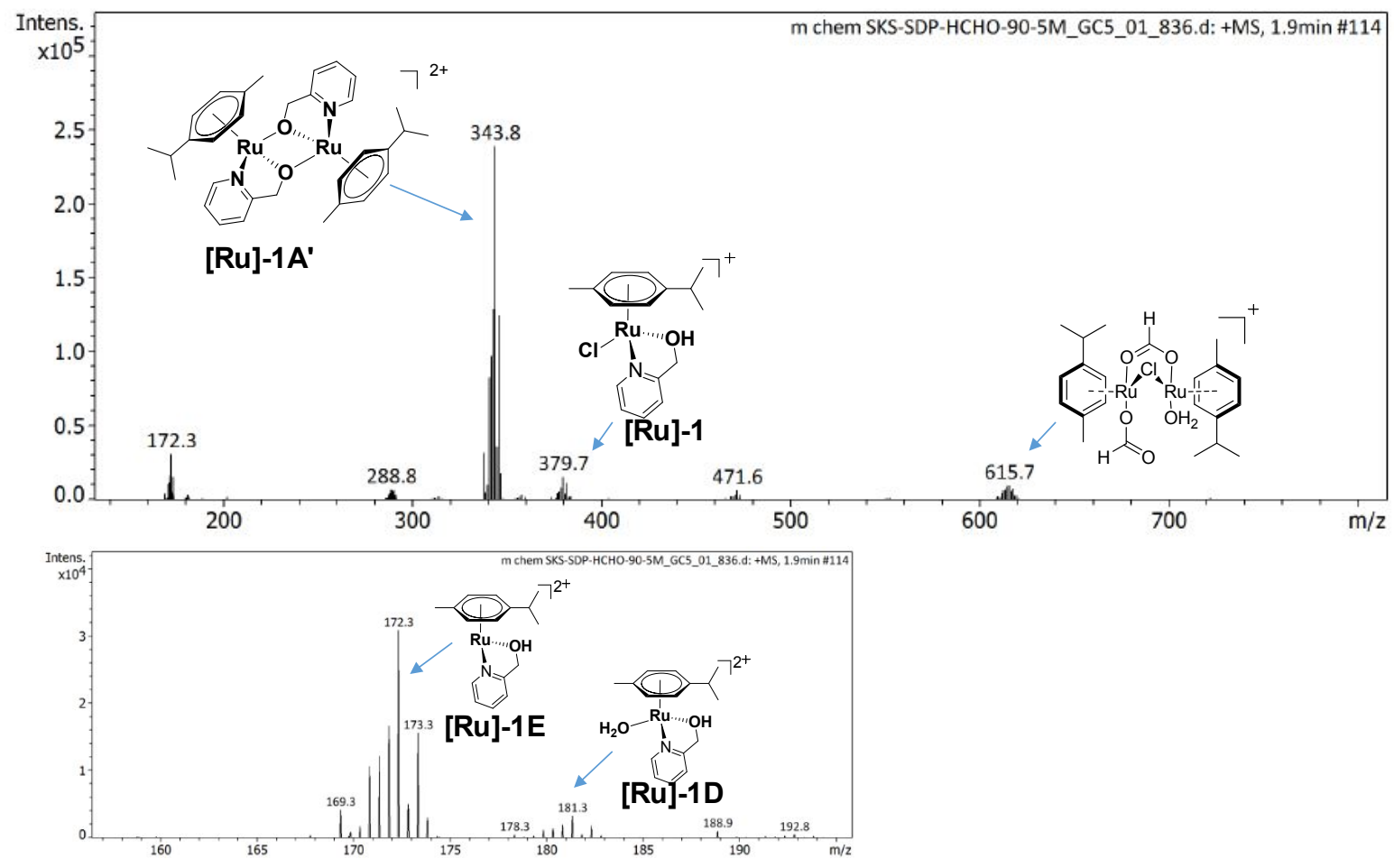

Figure S32. Mass spectra of the reaction aliquot the catalytic dehydrogenation of aq. formaldehyde after 5 minutes. Reaction condition: [Ru]-1 $(0.01 \mathrm{mmol})$, formaldehyde $(0.4 \mathrm{M}, 2.5 \mathrm{~mL}), \mathrm{T}=90^{\circ} \mathrm{C}$. 


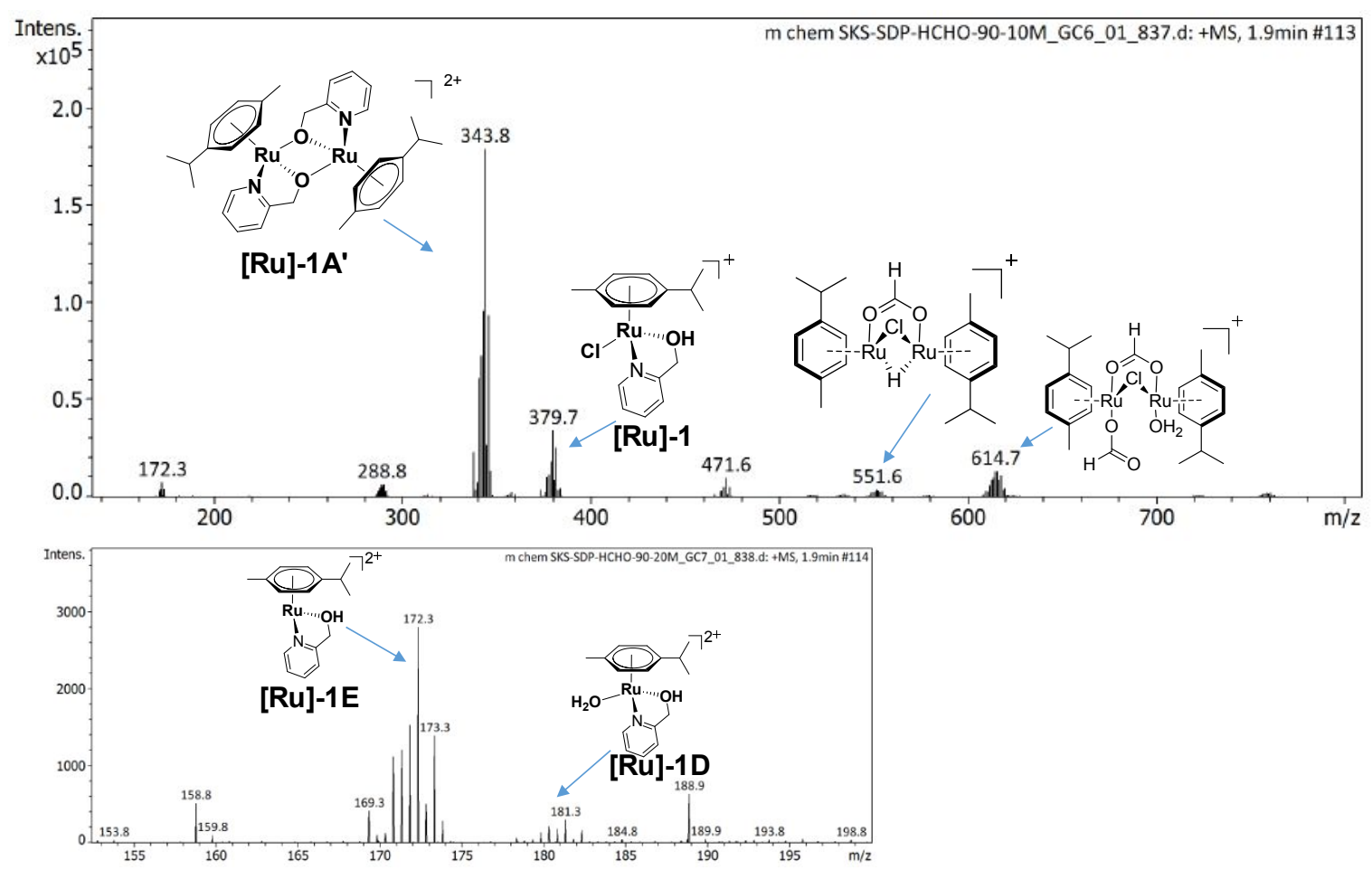

Figure S33. Mass spectra of the reaction aliquot for the catalytic dehydrogenation of aq. formaldehyde after 10 minutes. Reaction condition: $[R u]-1 \quad(0.01 \mathrm{mmol})$, formaldehyde $(0.4 \mathrm{M}, 2.5 \mathrm{~mL}), \mathrm{T}=90^{\circ} \mathrm{C}$.

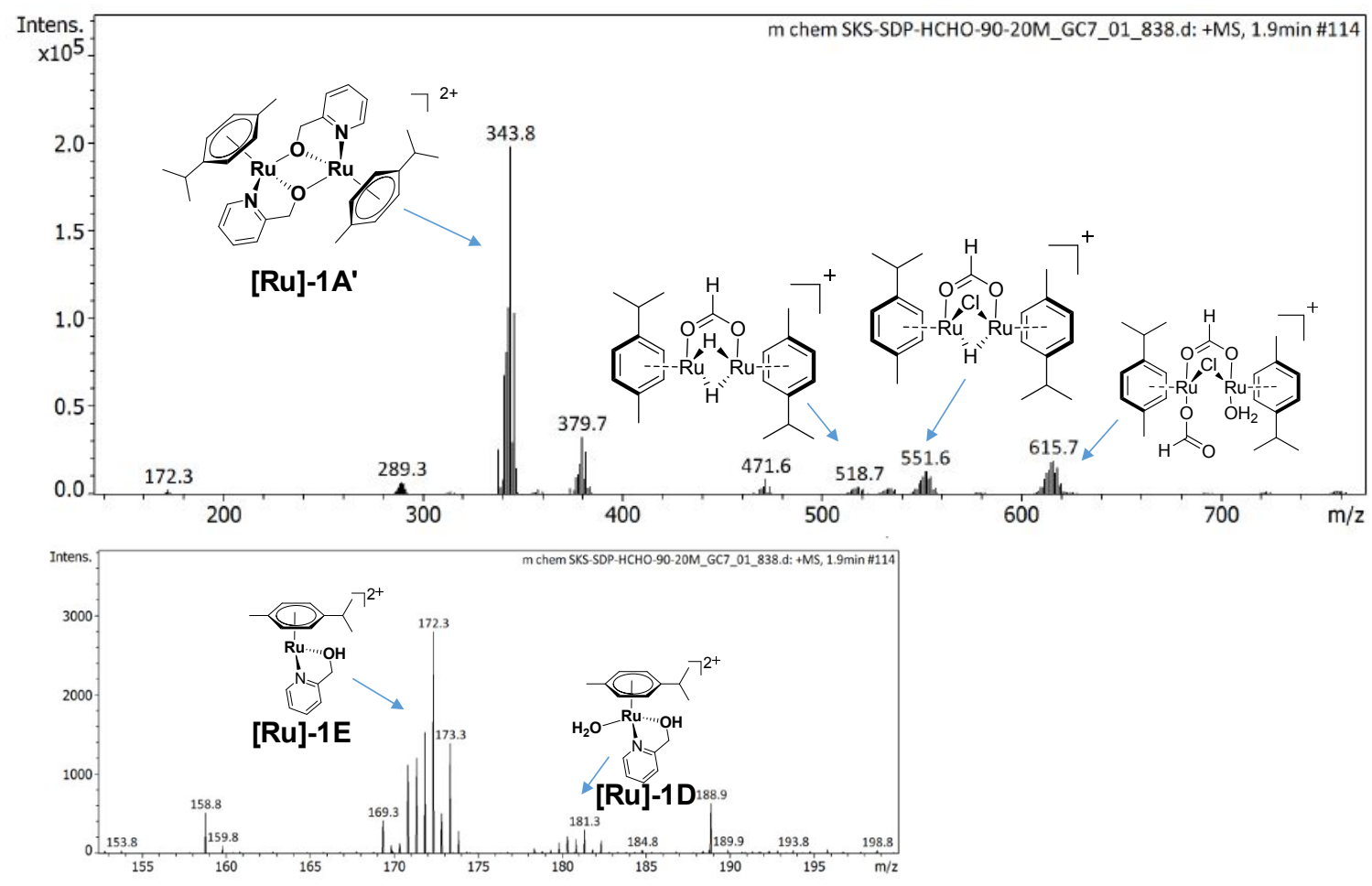

Figure S34. Mass spectra of the reaction aliquot for the catalytic dehydrogenation of aq. formaldehyde after 20 minutes. Reaction condition: [Ru]-1 (0.01 mmol), formaldehyde $(0.4 \mathrm{M}, 2.5 \mathrm{~mL}), \mathrm{T}=90^{\circ} \mathrm{C}$. 


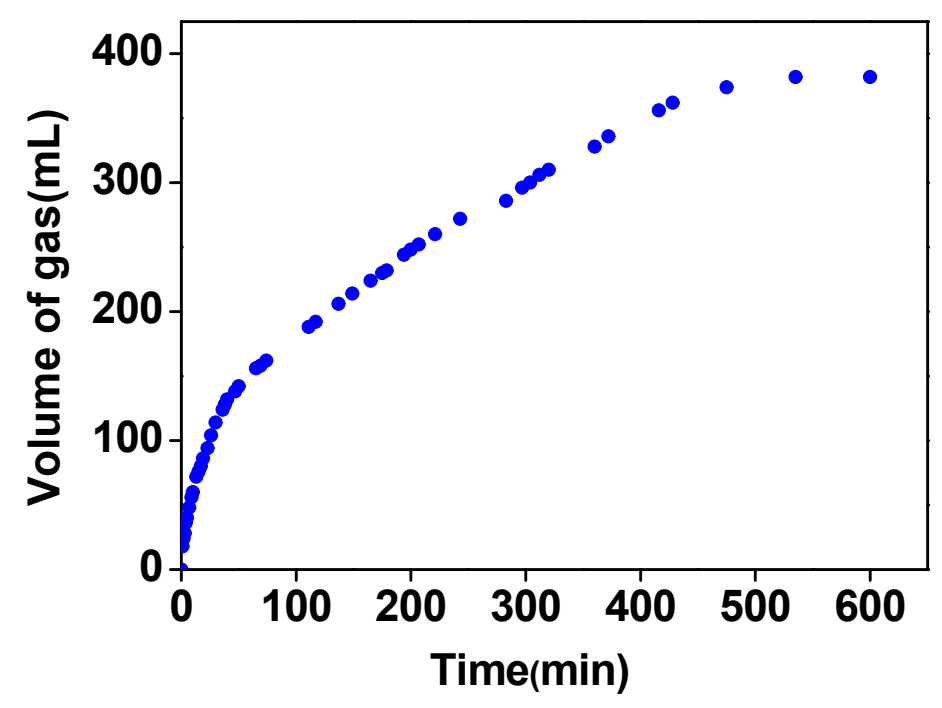

Figure S35. Time course plot for the catalytic dehydrogenation of aqueous formaldehyde $(13.55 \mathrm{mmol})$ over the catalyst $[\mathrm{Ru}]-1(0.1 \mathrm{~mol} \%), \mathrm{T}=95^{\circ} \mathrm{C}$ 
${ }^{1} \mathrm{H},{ }^{13} \mathrm{C}$ NMR and mass spectra of the ligands and complexes

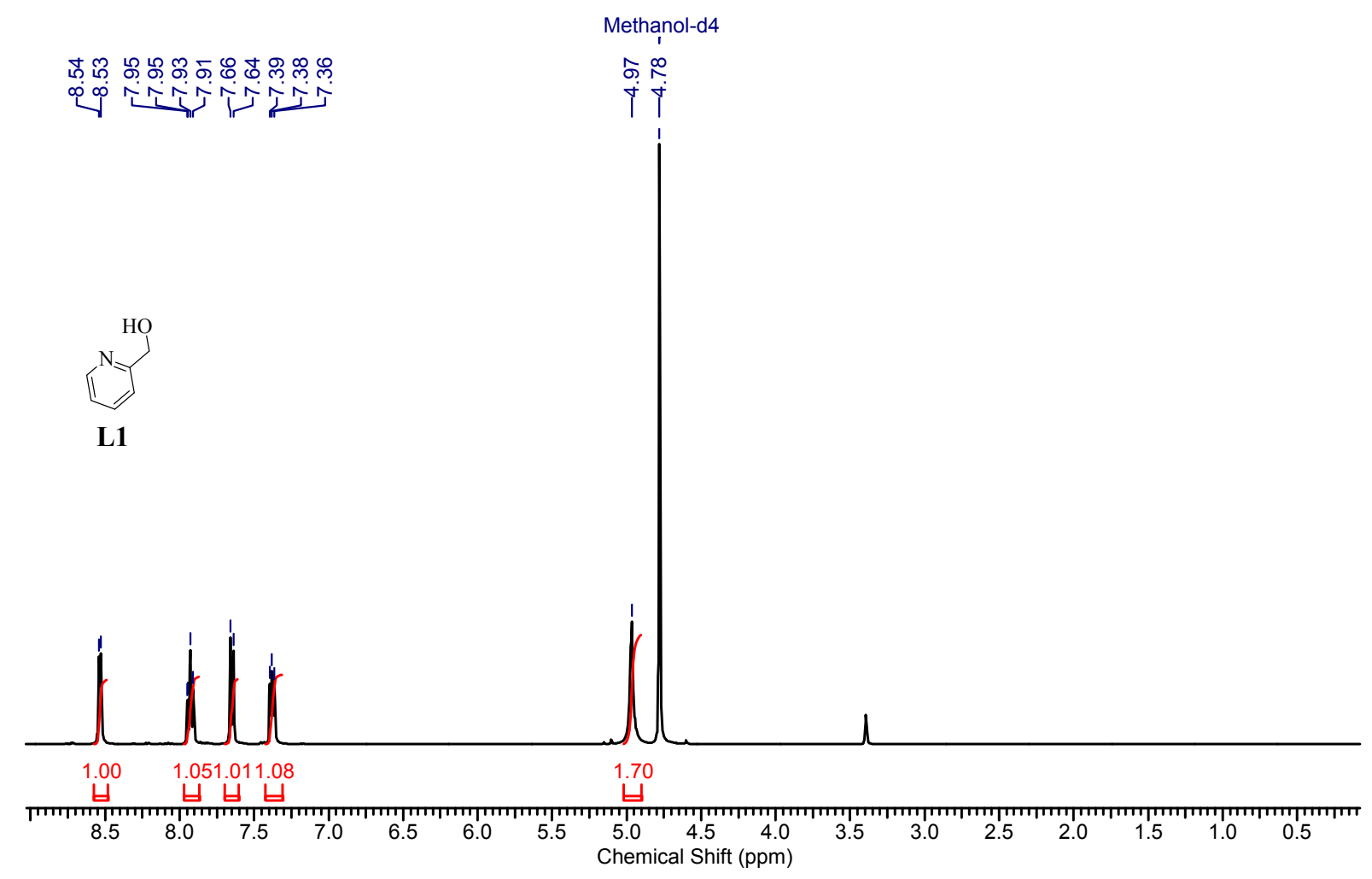

${ }^{1} \mathrm{H}$ NMR of $\mathbf{L} 1$ in $\mathrm{MeOH}-d_{4}$ 


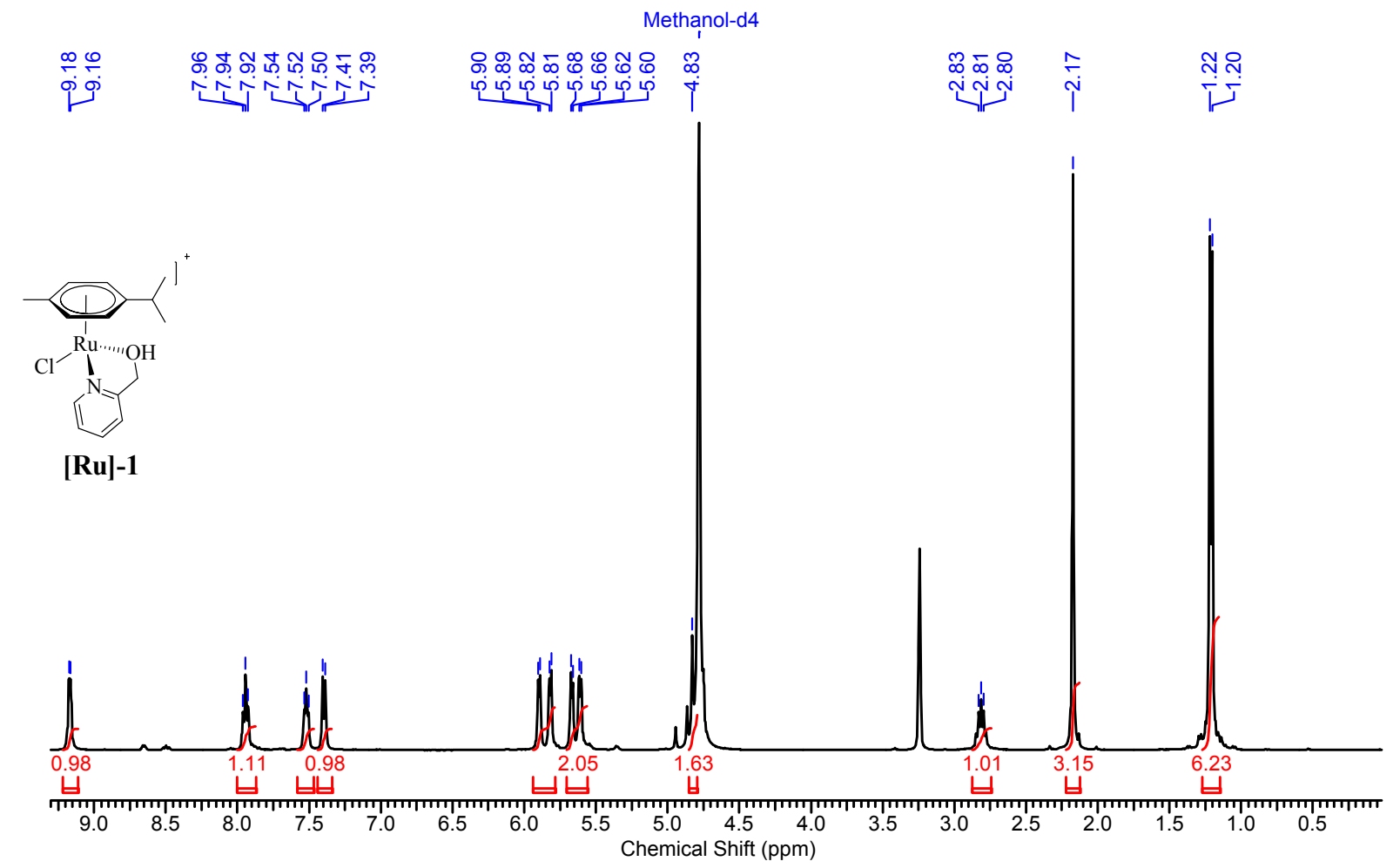

${ }^{1} \mathrm{H}$ NMR of $[\mathbf{R u}]-1$ in $\mathrm{MeOH}-d_{4}$

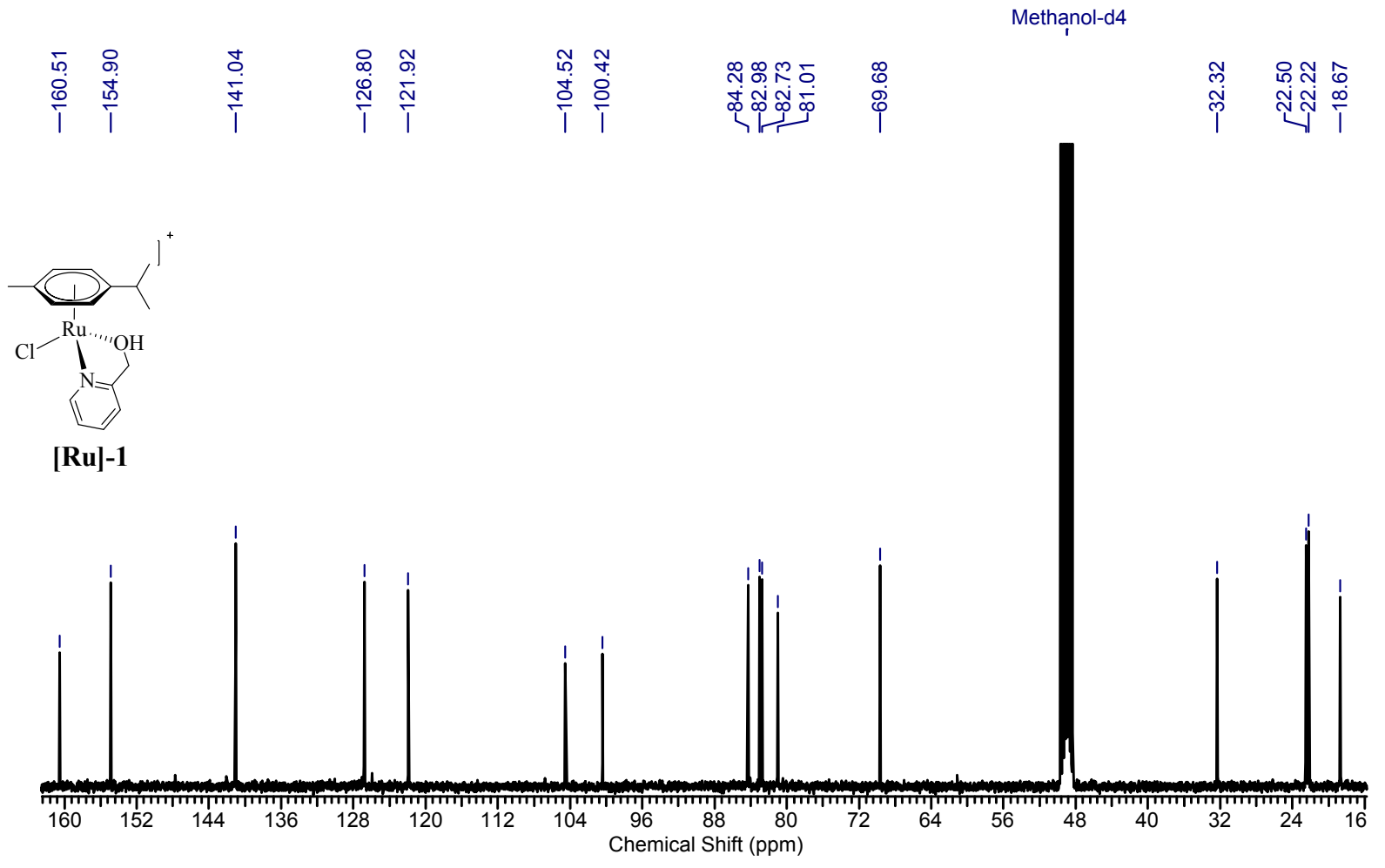

${ }^{13} \mathrm{C}$ NMR of $[\mathrm{Ru}]-1$ in $\mathrm{MeOH}-d_{4}$ 

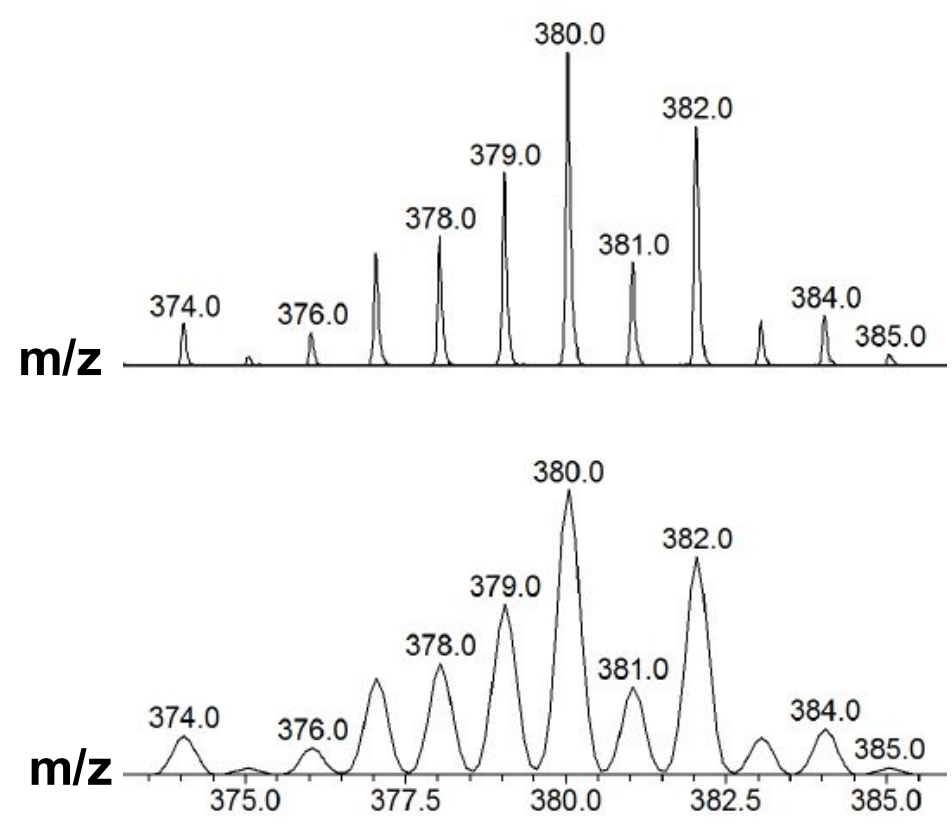

ESI-MS of [Ru]-1

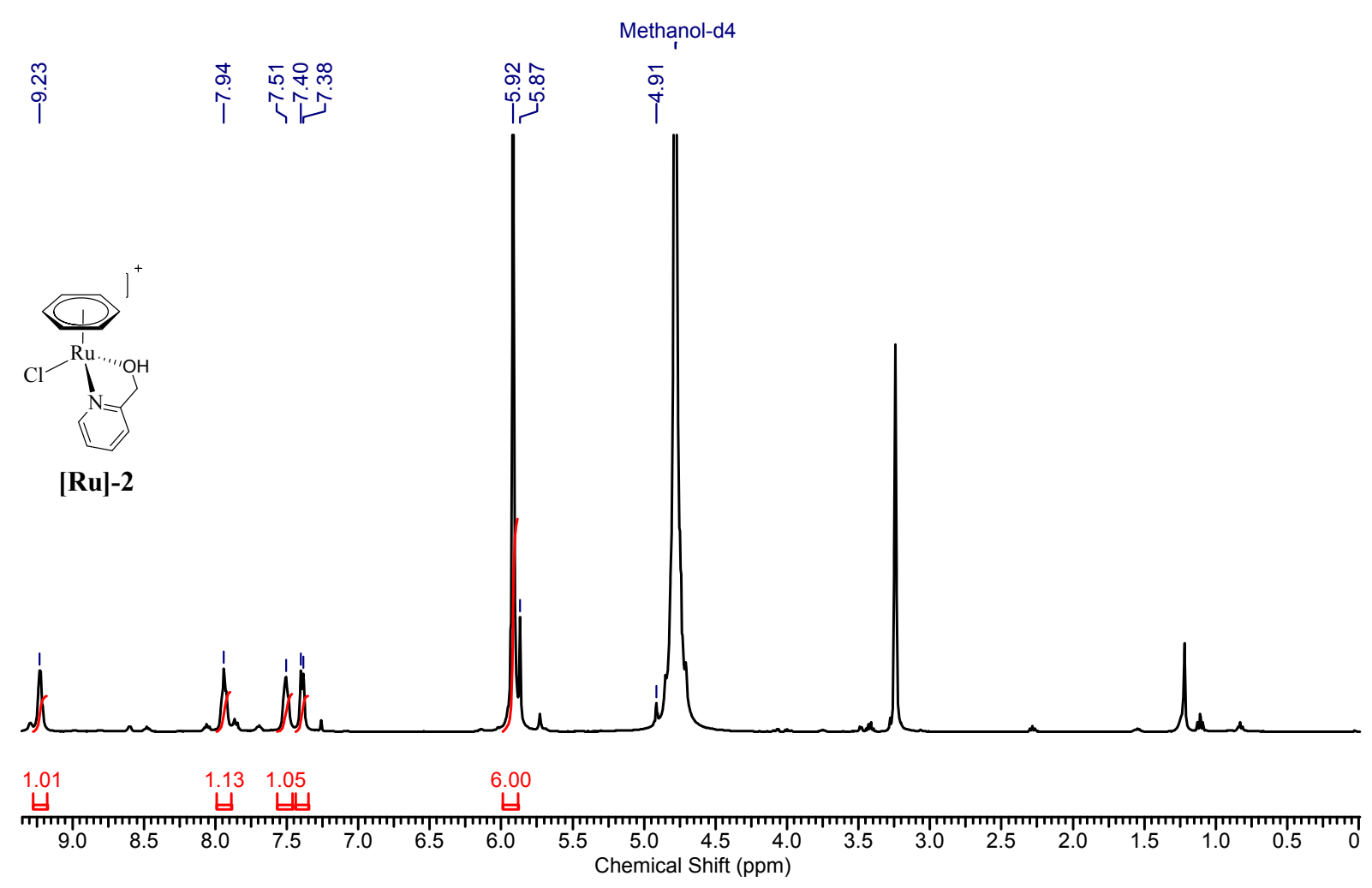

${ }^{1} \mathrm{H}$ NMR of [Ru]-2 in $\mathrm{MeOH}-d_{4}$ 

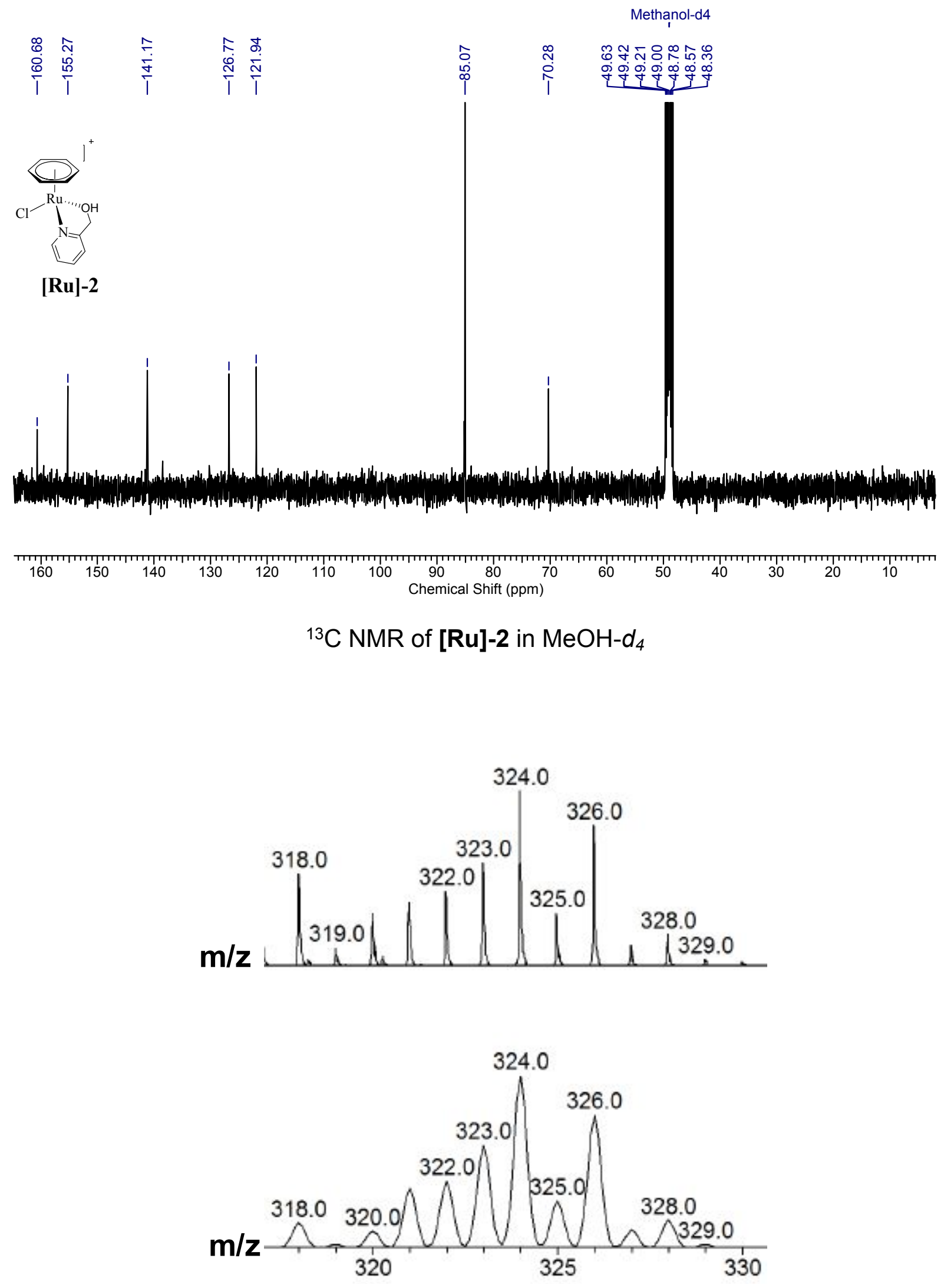

ESI-MS of [Ru]-2 
mల

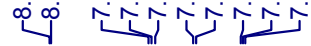

Methanol-d4

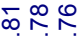

نं

$\stackrel{\text { }}{\text { p }}$

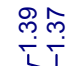

نَ

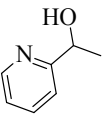

L2

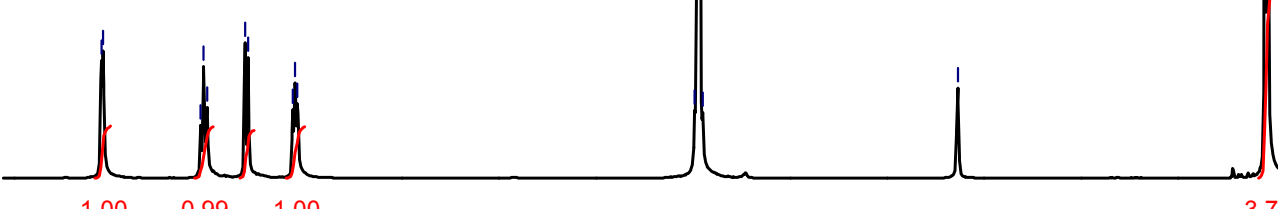

$\begin{array}{lll}1.00 & 0.99 & 1.00\end{array}$

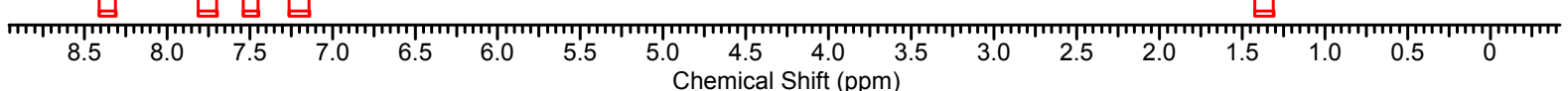

${ }^{1} \mathrm{H} \mathrm{NMR}$ of $\mathrm{L} 2$ in $\mathrm{MeOH}-d_{4}$

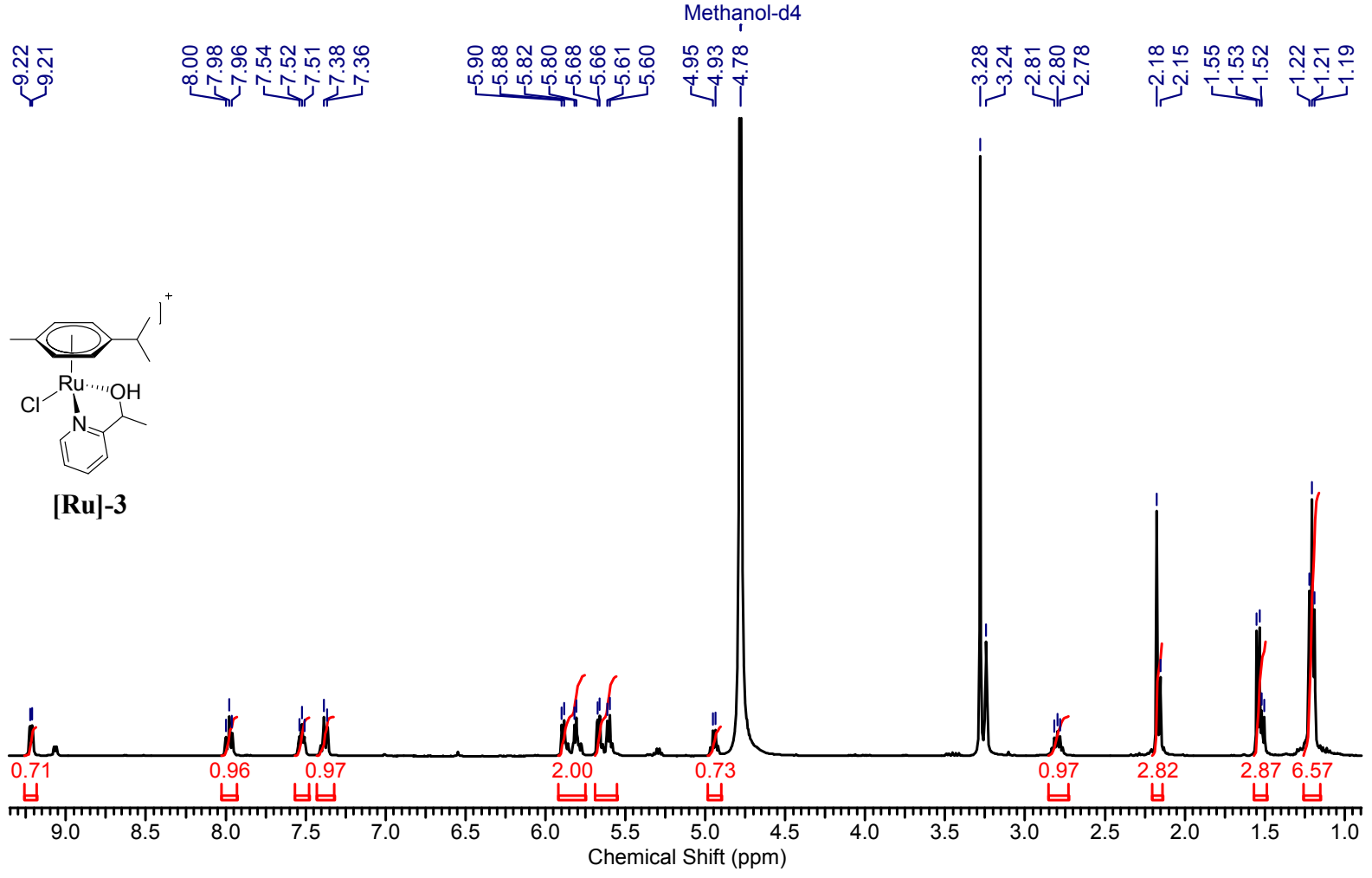

${ }^{1} \mathrm{H}$ NMR of $[\mathrm{Ru}]-3$ in $\mathrm{MeOH}-d_{4}$ 

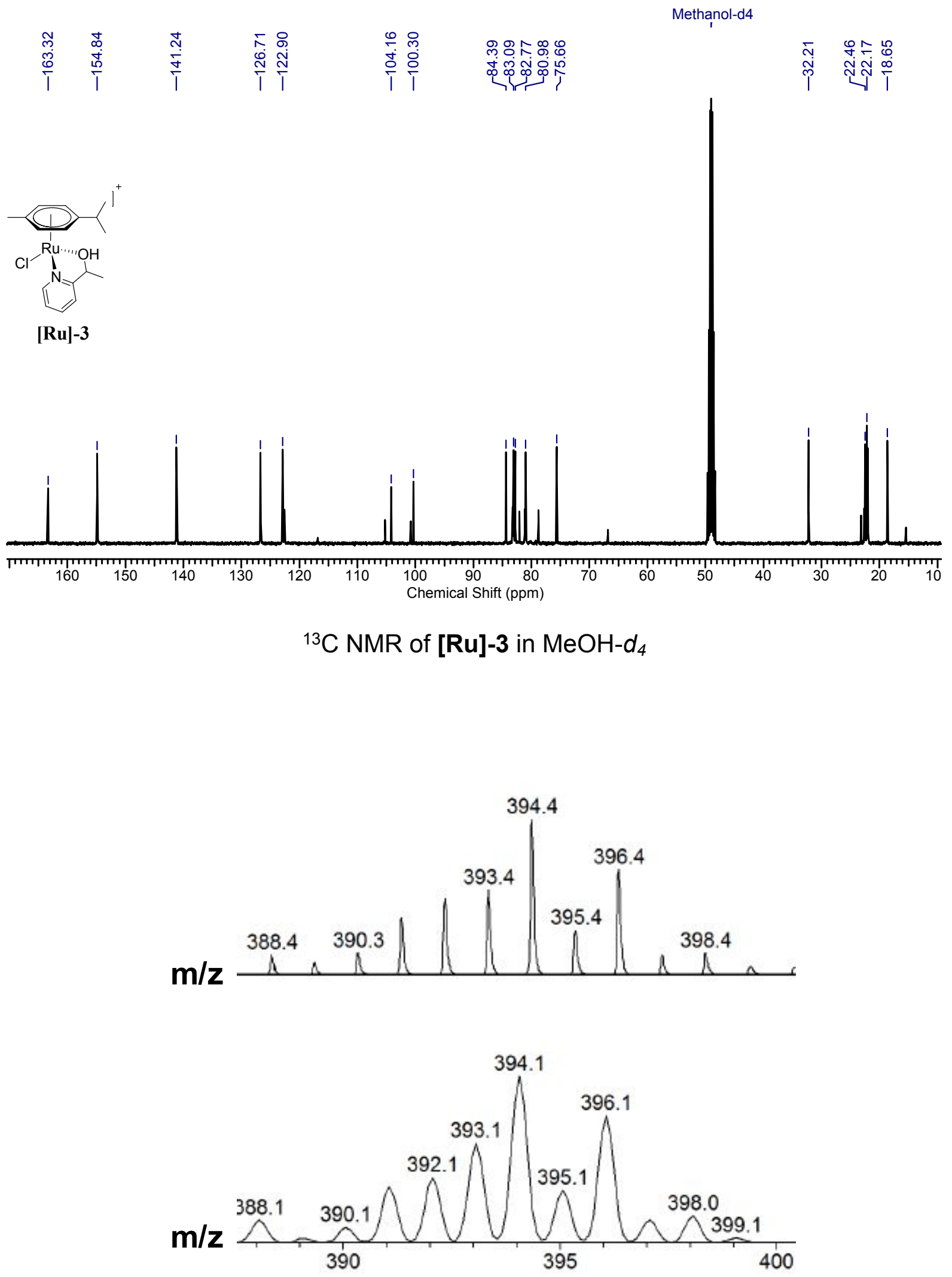

ESI-MS of [Ru]-3 


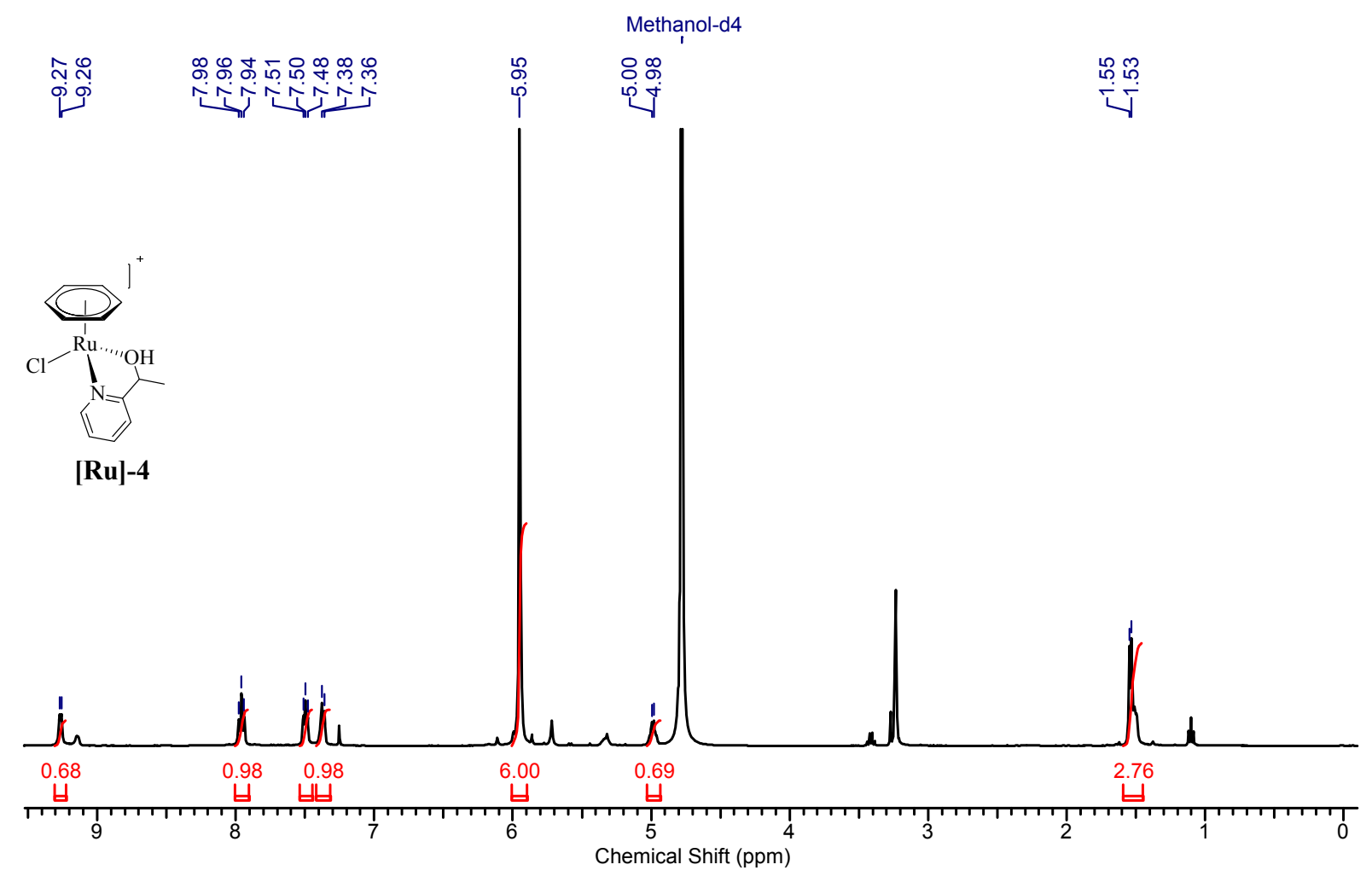

${ }^{1} \mathrm{H}$ NMR of $[\mathrm{Ru}]-4$ in $\mathrm{MeOH}-d_{4}$

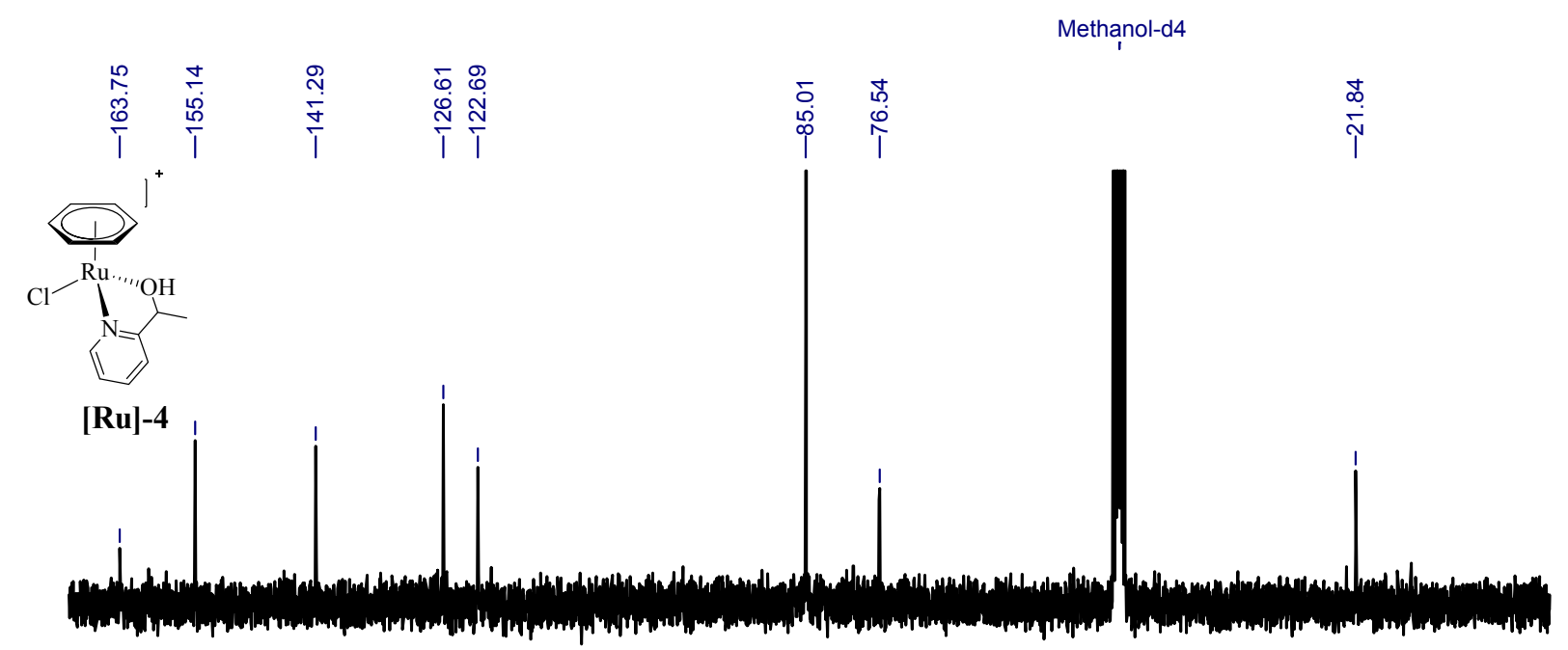

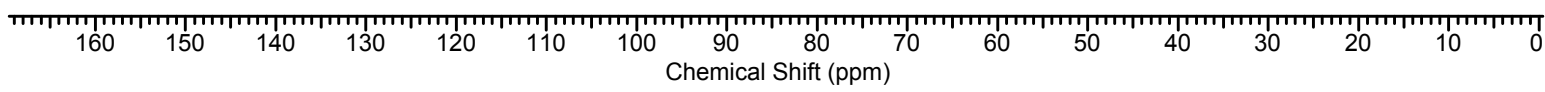

${ }^{13} \mathrm{C}$ NMR of $[\mathrm{Ru}]-4$ in $\mathrm{MeOH}-d_{4}$ 

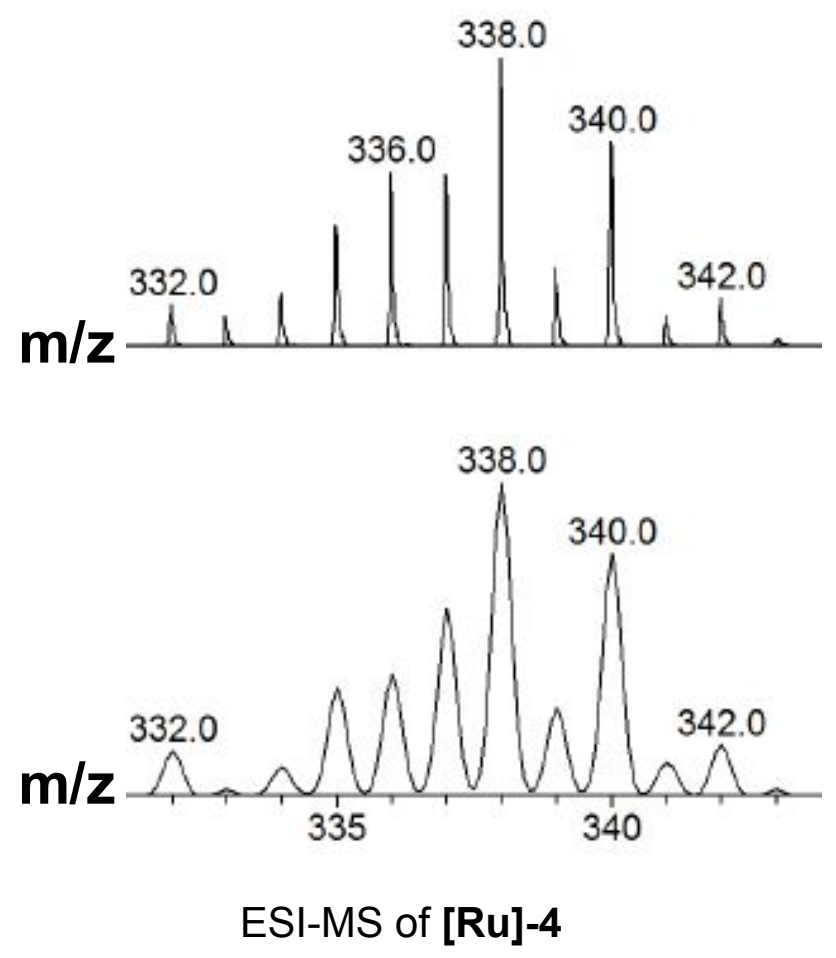

\section{References}

S1. (a) Patra, S.; Awasthi, M. K.; Rai, R. K.; Deka, H.; Mobin, S. M.; Singh, S. K. Dehydrogenation of Formic Acid Catalyzed by Water-Soluble Ruthenium Complexes: X-ray Crystal Structure of a Diruthenium Complex. Eur. J. Inorg. Chem.2019, 10461053. (b) Heim, L. E.; Schlörer, N. E.; Choi, J.-H.; Prechtl, M. H. G. Selective and Mild Hydrogen Production Using Water and Formaldehyde. Nat. Commun. 2014, 5, 3621 .

S2. Hull, J. F.; Himeda, Y.; Wang, W.-H.; Hashiguchi, B.; Periana, R.; Szalda, D. J.; Muckerman, J. T.; Fujita, E. Reversible hydrogen storage using $\mathrm{CO}_{2}$ and a protonswitchable iridium catalyst in aqueous media under mild temperatures and pressures. Nat. Chem. 2012, 4, 383.

S3. Himeda,Y. Highly efficient hydrogen evolution by decomposition of formic acid using an iridium catalyst with 4,4'-dihydroxy-2,2'-bipyridine. Green Chem. 2009, 11, 20182022.

S4. Manaka, Y.; Wang, W.-H.; Suna, Y.; Kambayashi, H.; Muckerman, J. T.; Fujita, E.; Himeda,Y. Efficient $\mathrm{H}_{2}$ generation from formic acid using azole complexes in water. Catal. Sci. Technol. 2014, 4, 34. 
S5. $\quad$ Wang, W. H.; Ertem, M. Z.; Xu, S.; Onishi, N.; Manaka, Y.;Suna, Y.;Kambayashi, H.; Muckerman, J. T.; Fujita, E.; Himeda, Y.Highly Robust Hydrogen Generation by Bioinspired Ir Complexes for Dehydrogenation of Formic Acid in Water: Experimental and Theoretical Mechanistic Investigations at Different pH.ACS Catal. 2015, 5, 54965504.

S6. Wang, W.-H.; Xu, S.; Manaka, Y.; Suna, Y.; Kambayashi, H.; Muckerman,J. T.; Fujita, E.; Himeda, Y. Formic Acid Dehydrogenation with Bioinspired Iridium Complexes: A Kinetic Isotope Effect Study and Mechanistic Insight. ChemSusChem2014, 7, 1976.

S7. Iguchi, M.; Himeda, Y.; Manaka, Y.;Kawanami, H.Development of an Iridium-Based Catalyst for High-Pressure Evolution of Hydrogen from Formic AcidChemSusChem. 2016, 9, 2749-2753.

S8. Wang, Z. J.; Lu, S. M.; Li, J.; Wang, J. J.; Li, C.; Unprecedentedly High Formic Acid Dehydrogenation Activity on an Iridium Complex with an N,N'-Diimine Ligand in Water. Chem. Eur. J. 2015, 21, 12592-12595.

S9. Lu, S. M.; Wang, Z.; Wang, J.; Li, J.; Li, C. Hydrogen generation from formic acid decomposition on a highly efficient iridium catalyst bearing a diaminoglyoxime ligand. Green Chem. 2018, 20, 1835-1840.

S10. Fellay, C.; Yan, N.; Dyson, P. J.; Laurenczy, G. Selective Formic Acid Decomposition for High-Pressure Hydrogen Generation: A Mechanistic Study. Chem. Eur. J. 2009, 15, 3752-3760.

S11. Loges, B.; Boddien, A.; Junge, H.; Beller, M. Controlled generation of hydrogen from formic acid amine adducts at room temperature and application in $\mathrm{H}_{2} / \mathrm{O}_{2}$ fuel cells. Angew. Chem., Int. Ed. 2008, 47, 3962-3965.

S12. Guan, C.; Zhang, D. D.; Pan, Y. P.; Iguchi, M.; Ajitha, M. J.; Hu, J. S.; Li, H. F.; Yao, C. G.; Huang, M. H.; Ming, S. X.; Zheng, J. R.; Himeda,Y.; Kawanami, H.; Huang,K. W. Dehydrogenation of Formic Acid Catalyzed by a Ruthenium Complex with an N,N'-Diimine Ligand. Inorg. Chem. 2017, 56, 438-445.

S13. Iturmendi, A.; Perez, L. R.; Perez- Torrente, J. J.; Iglesias, M.; Oro, L. A. Impact of Protic Ligands in the Ir-Catalyzed Dehydrogenation of Formic Acid in Water. Organometallics2018, 37, 20, 3611- 3618. 
S14. Fellay, C.; Dyson, P. J.; Laurenczy, G. A viable hydrogen-storage system based on selective formic acid decomposition with a ruthenium catalyst. Angew. Chem. Int. Ed. 2008, 47, 3966-3968.

S15. Boddien, A.; Federsel, C.; Sponholz, P.; Mellmann, D.; Jackstell, R.; Junge, H.; Laurenczy, G.; Beller,M. Towards the development of a hydrogen battery. Energy Environ Sci. 2012, 5, 8907-8911.

S16. Sponholz, P.; Mellmann, D.; Junge, H.; Beller, M. Towards a Practical Setup for Hydrogen Production from Formic Acid. ChemSusChem. 2013, 6, 1172-1176.

S17. Filonenko, G. A.; Putten, R. Van; Schulpen, E. N.; Hensen, E. J.; Pidko, E. A.Highly Efficient Reversible Hydrogenation of Carbon Dioxide to Formates Using a Ruthenium PNP-Pincer Catalyst. ChemCatChem. 2014, 6, 1526-1530.

S18. Pan, Y.; Pan, C. L.; Zhang, Y.; Li, H.; Min, S.; Guo, X.; Zheng, B.; Chen, H.; Anders, A.; Lai, Z.; Zheng, J.; Huang, K.-W. Selective Hydrogen Generation from Formic Acid with Well-Defined Complexes of Ruthenium and Phosphorus-Nitrogen PN3-Pincer Ligand. Chem. Asian J. 2016, 11, 1357-1360.

S19. Fukuzumi, S.; Kobayashi, T.; Suenobu, T. Efficient catalytic decomposition of formic acid for the selective generation of $\mathrm{H}_{2}$ and $\mathrm{H} / \mathrm{D}$ exchange with a water-soluble rhodium complex in aqueous solution. ChemSusChem 2008, 1, 827-834. 